Portland State University

PDXScholar

$1-1-2010$

\title{
3-D modelling of IC interconnect using OpenAccess and Art of Illusion
}

Navaneeth Prasannakumar Jamadagni

Portland State University

Follow this and additional works at: https://pdxscholar.library.pdx.edu/open_access_etds Let us know how access to this document benefits you.

\section{Recommended Citation}

Jamadagni, Navaneeth Prasannakumar, "3-D modelling of IC interconnect using OpenAccess and Art of Illusion" (2010). Dissertations and Theses. Paper 28.

https://doi.org/10.15760/etd.28

This Thesis is brought to you for free and open access. It has been accepted for inclusion in Dissertations and Theses by an authorized administrator of PDXScholar. Please contact us if we can make this document more accessible: pdxscholar@pdx.edu. 
3-D Modelling of IC Interconnect using OpenAccess and Art of Illusion

by

Navaneeth Prasannakumar Jamadagni

A thesis submitted in partial fulfillment of the requirements for the degree of

\author{
Master of Science \\ in \\ Electrical and Computer Engineering
}

Thesis Committee:

Paul Van Halen, Chair, Branimir Pejcinovic

Richard Campbell

Portland State University

(C) 2010 


\begin{abstract}
In search of higher speed and integration, the integrated circuit (IC) technology is scaling down. The total on-chip interconnect length is increasing exponentially. In fact, interconnect takes up the most part of the total chip area. The parasitics associated with these interconnect have significant impact on the circuit performance. Some of the effects of parasitics include cross talk, voltage drop and high current density. These issues can result in cross-talk induced functional failure and failures due to IR drop and electro-migration. This has resulted in interconnectdriven design trend in state-of-the-art integrated circuits. Reliability analysis, that includes simulating the effects of parasitics for voltage drop, current density, has become one of the most important steps in the VLSI design flow. Most of the CAD/EDA tools available, map these analysis results two dimensionally. Although this helps the designer, providing a three dimensional view of these results is highly desirable when dealing with complex circuits.

In pursuit of visualizing reliability analysis results three dimensionally, as a first step, this work presents a tool that can visualize IC interconnect three dimensionally. Throughout the course of this research open source tools were used to achieve the objective. In this work the circuit layout is stored as an OpenAccess database. $\mathrm{A} \mathrm{C}++$ program reads the design information using OpenAccess API and converts it to the .OBJ file format. Art of Illusion, an open source 3D modeling and rendering tool, reads this .OBJ file and models the IC interconnect three-dimensionally. In addition, Eclipse, an open source java IDE is used as a development platform. The tool presented has the capability to zoom in, zoom out and pan in real time.
\end{abstract}




\section{Acknowledgements}

I am indebted to Dr. Paul Van Halen for his advice and suggestions throughout the course of this research. There were numerous meetings and discussions that led to successful completion of this thesis. I am also grateful to the committee members, Dr. Branimir Pejcinovic and Dr. Richard Campbell for reviewing this document and suggesting key changes.

Most importantly, I am thankful to my parents, my sister and all my friends for their continued moral support and encouragement. 


\section{Contents}

$\begin{array}{ll}\text { Abstract } & \text { i }\end{array}$

Acknowledgements $\quad$ ii

List of Tables vii

List of Figures viii

1 INTRODUCTION 1

2 3D Modeling of Interconnects 4

2.1 Motivation to Model 3 Dimensionally . . . . . . . . . . . . . 4

2.2 An Open Source Platform for Tools Integration and Deployment . . 8

2.3 Modeling IC Interconnect three dimensionally . . . . . . . . . . . . 9

3 Previous Work 13

4 OpenAccess 16

4.1 Need for Tools Interoperability . . . . . . . . . . . . . . . 16

4.2 API for Interoperability . . . . . . . . . . . . . . . 18

4.3 OpenAccess . . . . . . . . . . . . . . . . . . . . 21

4.3.1 Features of OpenAccess . . . . . . . . . . . . . . 22 
4.3.2 Features of the OpenAccess Reference Implementation . . . 23

4.3.3 OpenAccess Organization ............. . . 24

4.3.4 Limitations of OpenAccess . . . . . . . . . . . . . . . 27

5 Integration of $3 \mathrm{D}$ viewer with OpenAccess 31

5.1 Art of Illusion . . . . . . . . . . . . . . . . . . . 31

5.2 Integration of Art of Illusion with OpenAccess . . . . . . . . . . . 32

5.2.1 Extending Art of Illusion . . . . . . . . . . . . . . . 32

5.2 .2 Application Integration with OpenAccess . . . . . . . . . 33

5.2 .3 Java Native Interface and SWIG . . . . . . . . . . . . 35

6 Query Implementation $\quad 37$

6.1 OpenAccess API Classes . . . . . . . . . . . . . . . 37

6.1.1 Managed Classes . . . . . . . . . . . . . . . . 38

6.1 .2 Utility Classes . . . . . . . . . . . . . . . . . . 39

6.1.3 Interface Classes . . . . . . . . . . . . . . . . . . 39

6.2 Query Algorithm ................... 41

6.2 .1 LPP Query .................... 42

6.2.2 Shape Query .................. 44

6.3 Query Pseudocode ...................... 47

7 Polygons to Rectangles $\quad 48$

7.1 PTR Algorithm . . . . . . . . . . . . . . . . . 49

7.2 Modified PTR, m-PTR Algorithm . . . . . . . . . . . . . 51

$82 \mathrm{D}$ to $3 \mathrm{D}$ conversion $\quad 54$

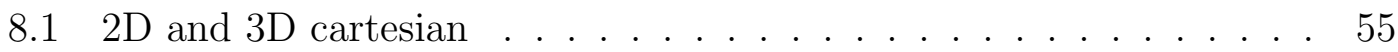


8.2 .OBJ and .MTL file formats . . . . . . . . . . . . . . 58

8.2 .1 .OBJ File Format . . . . . . . . . . . . . . 58

8.2 .2 .MTL File Format . . . . . . . . . . . . . . . . . . . . 59

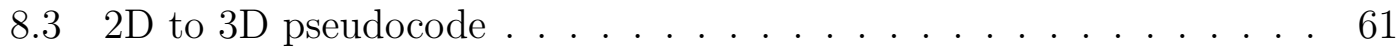

9 Eclipse Platform for Tool Integration and Deployment $\quad 63$

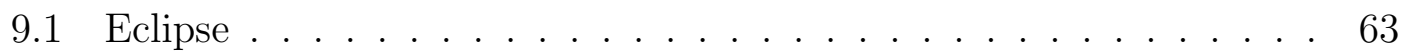

9.2 Eclipse for Tools Integration . . . . . . . . . . . . . . . . . . 64

9.3 Eclipse for Tool Deployment . . . . . . . . . . . . . . . 67

10 Test results and discussion $\quad 69$

10.1 Limitations of the Algorithm . . . . . . . . . . . . . 78

$\begin{array}{ll}11 \text { Future work } & 79\end{array}$

11.1 Extending OA Database ................. . . 79

11.2 Integration of Parasitic extraction and simulation tools . . . . . . . 80

12 Conclusion $\quad 83$

$\begin{array}{lr}\text { References } & 86\end{array}$

Appendices

$\begin{array}{lll}\text { A .OBJ File Format } & 91\end{array}$

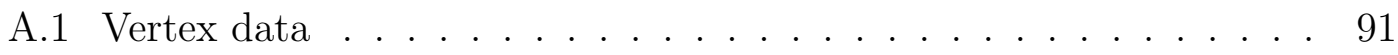

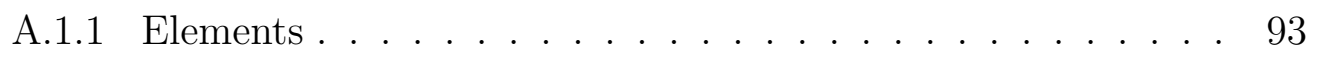

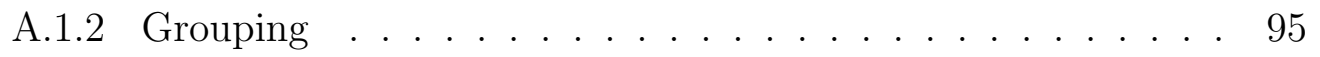

A.1.3 Display/Render attributes . . . . . . . . . . . . . . 96 
$\begin{array}{ll}\text { B .MTL File Format } & 98\end{array}$

C Importing OpenAccess to Eclipse 101

C.1 Installing TCL wrappers . . . . . . . . . . . . . . . . . . 102

D Importing Art of Illusion to Eclipse 104

$\begin{array}{ll}\text { E Schema Diagram Legend } & 105\end{array}$ 


\section{List of Tables}

5.1 Overview of OpenAccess API Functions and Classes . . . . . . . . . 35

6.1 Utility classes and examples[1] . . . . . . . . . . . . . . . . 39

6.2 Interface Classes and OA Class Integration $[1] \ldots \ldots \ldots$

7.1 Comparison of PTR and m-PTR algorithm for different polygons . 52

10.1 Time to write the .OBJ file $\ldots \ldots \ldots \ldots \ldots \ldots$ 


\section{List of Figures}

1.1 Interconnect and Gate delays in different process technologies . . . 2

2.1 EM, IR drop and Signal Integrity analyses flow $\ldots \ldots \ldots \ldots$

2.2 IR drop violation modelled in Cadence Voltagestorm . . . . . . 8

2.3 Tools necessary to visualize EM and IR analyses results three di-

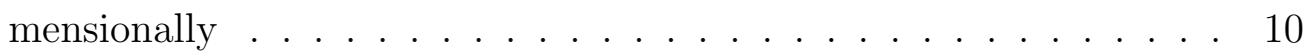

2.4 Two ways to extend applications capability to read different data

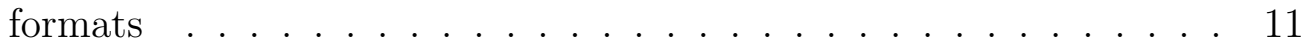

3.1 3D IC Layout viewer by Sicard $[2] \ldots \ldots \ldots \ldots$

3.23 D view of an Inverter using VRML model[3] . . . . . . . . . 14

3.3 Data Flow in Jale3D[4] . . . . . . . . . . . . . . . 15

4.1 Tools used in Basic Analog/Mixed Signal Design Flow . . . . . . 17

4.2 Design Exchange Using LEF and DEF . . . . . . . . . . . . 19

4.3 Design Exchange using an API . . . . . . . . . . . 20

4.4 OpenAccess - API Packages . . . . . . . . . . . . . . . 24

4.5 OpenAccess - Design data organization . . . . . . . . 26

4.6 Schema Diagram - oaDesign Class $[5] \ldots \ldots \ldots \ldots$

4.7 Schema Diagram - oaTech class $[5] \ldots \ldots \ldots$ 
5.1 Extending Art of Illusion functionality . . . . . . . . . . . . . 33

5.2 Application Integration with OpenAccess . . . . . . . . . . . . 34

6.1 Managed Classes[1] . . . . . . . . . . . . . . . . . . 39

6.2 Layer-Purpose-Shape Relationship . . . . . . . . . . . . . . . . . . . 41

6.3 Query Algorithm .................. 43

6.4 Information Model of oaShape class . . . . . . . . . . . . . . . . 45

7.1 Example of PTR algorithm[6] . . . . . . . . . . . . 50

7.2 Partitioning Polygon to Rectangles using the m-PTR algorithm . . 51

7.3 Polygon Examples . . . . . . . . . . . . . . . . . . . 53

8.1 Figure showing $2 \mathrm{D}$ and 3D cartesian . . . . . . . . . 56

8.2 Computing layer thickness . . . . . . . . . . . . . . . 57

8.3 A 3D rectangle, modeled in Art of Illusion . . . . . . . . . . . . . . 60

9.1 Possible outcome of Integration in Eclipse Framework . . . . . . . . 66

10.1 Complete Algorithm Flow Chart . . . . . . . . . . . . . . 71

10.2 Complete Algorithm Flow Chart cont. . . . . . . . . . . . . . . . . 72

10.3 Top view of Nand Gate in oaViewer and Art of Illusion . . . . . . . 74

10.4 Corrected Z-Axis Top View . . . . . . . . . . . . . . . . 75

10.5 Full Adder Cell Viewed in oaViewer and Art of Illusion . . . . . . . 76

10.6 3D view of Full Adder interconnect . . . . . . . . . . . . . . 77 


\section{Chapter 1}

\section{INTRODUCTION}

Physical layout design is an important phase in the VLSI design flow. Layout design is an art and it requires careful attention to detail, as it determines the overall performance of the circuit. The gate length of an active device like Metal Oxide Semiconductor Field-Effect Transistor (MOSFET) is ever shrinking and it is in the range of 32 to $45 \mathrm{~nm}$. In general the industry scales process technology with scaling factor, $S=\sqrt{2}[7]$, i.e, the next generation of the chips will have twice the number of transistors per unit area than its predecessor. Smaller devices are faster, increasing the speed of the circuit. Higher device density allows integrating more functionality in a single chip. Although this is highly desirable, interconnecting these devices with metal wires takes up a greater percentage of the area. The metal wires are referred to as interconnect.

With technology scaling, the width of interconnect reduces, but the length of the interconnect increases exponentially, increasing its resistance $(R)$, capacitance (C) and in some cases inductance (L). Hence increasing the delay in interconnect. In fact, interconnect delay has become the dominant source of circuit delay in geometries less than 250nm, as shown in the Fig.1.1. Moreover higher capacitance in interconnect increases the switching power. 
Traditionally, Aluminum $(\mathrm{Al})$ and its alloys have been the choice of material for interconnect. Copper $(\mathrm{Cu})$, because of its lower sheet resistance, is replacing $\mathrm{Al}$ as the material for interconnect. Most designs using 180nm process technology and beyond, uses $\mathrm{Cu}$ for interconnect. Yet, the interconnect delay remains dominant. Parasitics associated with interconnect also presents reliability issues like electromigration and voltage drops. This along with Signal Integrity issues will be discussed briefly in section 2.1. Therefore, designing and analyzing interconnect have become as important as device engineering.

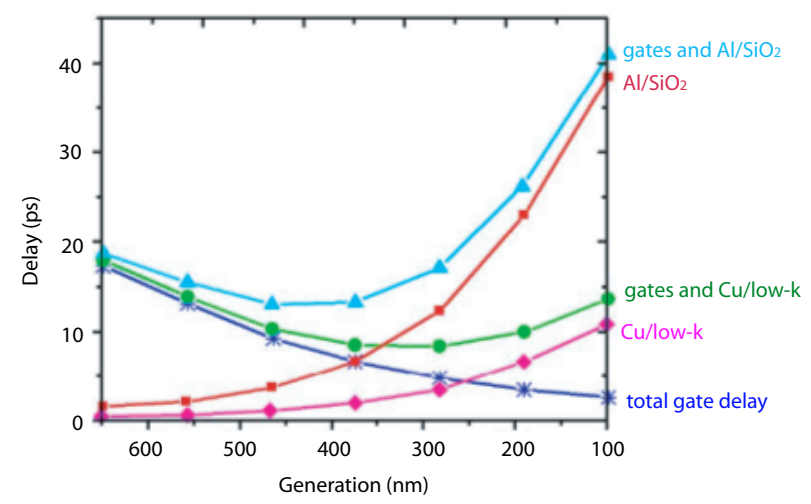

Figure 1.1: Interconnect and Gate delays in different process technologies $[8]$

The research presented in this document, aims at modeling the IC interconnect three dimensionally. Providing the designer with an extra dimension to view, helps the designer to understand the impact of interconnect on circuit's performance. This document begins by presenting the current flow for reliability analyses, similar work implemented and their limitations. In the subsequent sections usage of open source tools to realize the objective, challenges involved and their limitations are discussed. 
The industry and academic research uses CAD/EDA tools from different vendors, in their design flow. Using different tools implies different data formats and conversion between data formats is required. This conversion is prone to error, increasing the design cycle time. Thus, a more data centric approach is needed in the flow. To address this issue Si2 Inc., has provided an open source API to a design database, called OpenAccess. This work uses the OpenAccess based design.

Art of Illusion, an open source 3D modeling and rendering tool, is used to render IC interconnect three dimensionally. Throughout the course of this research Eclipse, an open source tool that provides integrated development and deployment environment, is used. In this research Eclipse is mainly used as a development environment. A study on Eclipse as an integration and deployment platform is also presented in the chapter 9. The work makes use of Nangate's OpenCell 45nm standard cells to demonstrate the modeling of interconnect three dimensionally and Synopsys OA viewer is used to verify the results. 


\section{Chapter 2}

\section{D Modeling of Interconnects}

\subsection{Motivation to Model 3 Dimensionally}

Continuing advancements in the process technology has allowed aggressive scaling of the device feature size. The technology scaling has steadily improved the circuit performance. Since the area consumption is less, more functional units are integrated on the same chip. However, due to noise immunity issues, the supply voltage hasn't scaled accordingly. Transistor and interconnect scaling without appropriate voltage scaling results in high power density. This coupled with high frequency design trend increases the risk of joule heating and electromigration(EM). Electromigration is a thermal activity and is aggravated by joule heating. Joule heating, $\mathrm{Q}$, is directly proportional to the square of the current in the wire, I, multiplied by the resistance of the wire, $\mathrm{R}$ i.e, $Q \propto I^{2} * R$. Therefore high current densities and interconnect scaling exacerbates electro-migration, eventually leading to chip failure.

Interconnect has electrical resistance associated with it. Due to this resistance, there exists voltage drop in the network comprising interconnect. The voltage drop due to interconnect resistance is commonly called IR drop. Scaling of interconnect increases its resistance, resulting in higher voltage drop. Excessive voltage drop in 
the supply grid reduces the available supply voltage at transistor contacts. This degrades the switching speed and noise margin of transistors resulting in functional failure.

Clearly, from the above discussion, both IR drops and EM degrades the reliability of the chip. Electro-migration can be alleviated by using a different interconnect material and limiting the current density. From a designer's perspective modifying the design to limit the current density is more practical and achievable than using a different material. Similarly, IR drops in the interconnect can be minimized by routing signals in different layer and adding decoupling capacitors at strategic points to reduce supply voltage drop. Raychaudhuri et al. [9] shows that the vias dominate the impedance in the interconnect network and insufficient number of vias tend to be the root cause of failures due to IR drop. Adding more vias at critical junctions can reduce such a failure. Hence most design flows incorporate EM and IR drop analysis. In fact, the article published by Cadence [10] shows that EM and IR drop analysis are among the top ten things that the designers need for the tape-out.

Parasitics in the interconnect also presents signal integrity(SI) issues like ringing, cross talk, ground bounce and supply noise. Cross talk and noise are the major concerns for a designer. The primary source of cross talk is coupling due to parasitic capacitance in the interconnect. Other sources of cross talk include mutual inductance and substrate coupling. Analog designers are more concerned with the substrate noise coupling, especially in an analog mixed signal environment, where noisy digital signals effect sensitive analog signals. Possible solutions for signal integrity issues include buffer insertion, wire shielding, routing changes etc. With all these issues, i.e., EM, IR drops and Signal Integrity, engineering 
the interconnect has become as equally important as engineering the transistors that sit underneath them. This has made parasitic extraction one of the most important phases of a design cycle in all signal domains, i.e., Analog, Digital and RF.

Accurately modeling the impact of the parasitics for EM, IR drops and SI and back annotating the results and visualizing the simulated data, helps the designer to identify and fix the problem. The circuit can be analyzed for electromigration and IR drop by looking at the current density and voltage distributions respectively. Timing analysis is usually performed to check for signal integrity. Fortunately there are CAD tools available from different vendors to analyze the reliability of the circuit.

Figure 2.1 shows the current design flow for Electro Migration, IR Drop and Signal Integrity analysis. Also shown in the figure are the Cadence tools used in the design flow. Similar tools are also available from Synopsys, Mentor Graphics and Ansoft.

Figure 2.2 shows the design with voltage drop problems modeled in Cadence Voltagestorm tool. Most of the tools model the current density and voltage distributions two dimensionally and generates a huge text file that contains analysis report for each net. Modeling the analysis data two dimensionally only helps the designer to localize the problem and requires getting more data from the text file, which can be cumbersome. It will be much more beneficial for a designer, if the current density and voltage distributions are modeled three-dimensionally with capability to zoom in to a particular node or a via. This helps the designer to locate the troublesome net or node and solve the problem faster, thereby reducing the design cycle time. 


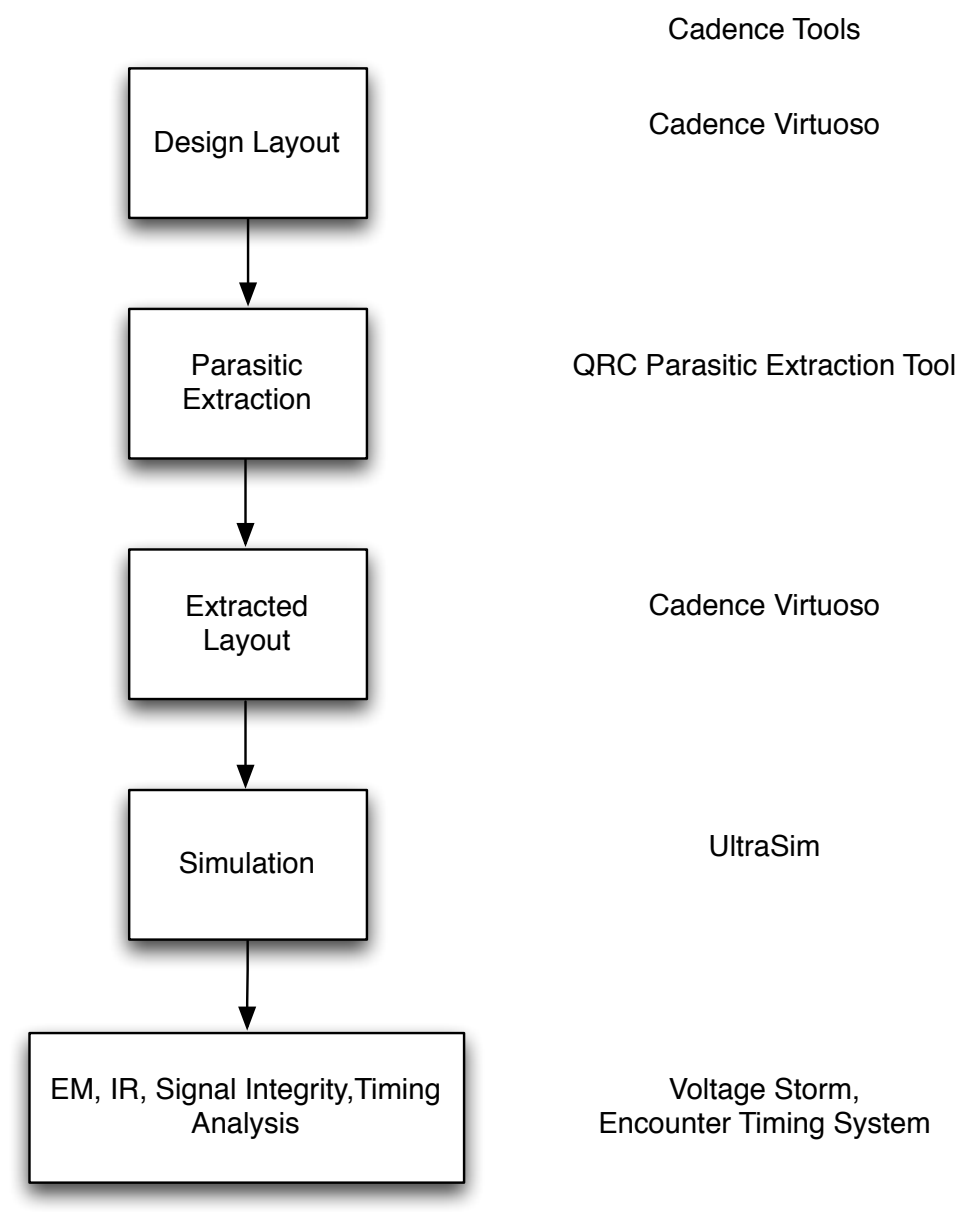

Figure 2.1: EM, IR drop and Signal Integrity analyses flow 

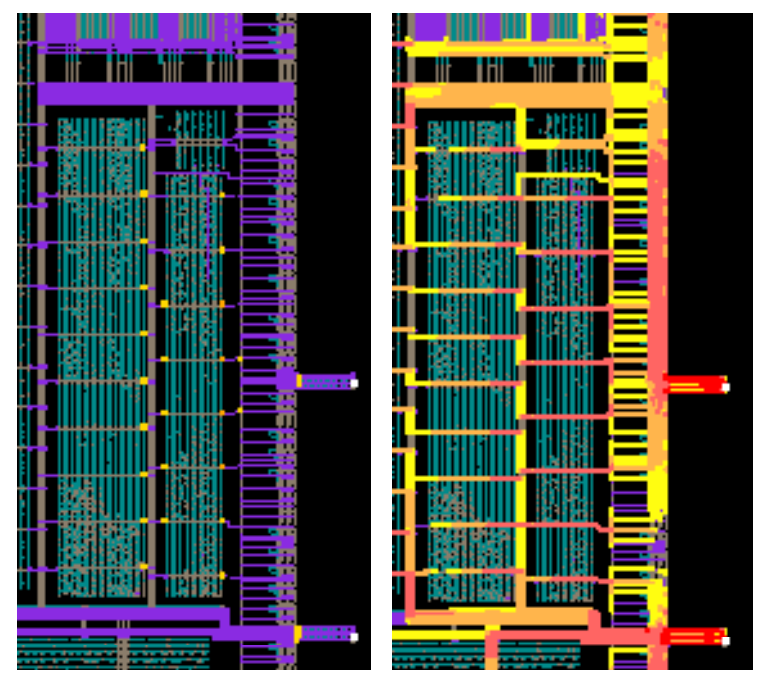

Figure 2.2: IR drop violation modelled in Cadence Voltagestorm $[10]$

Rendering such a huge amount of data three-dimensionally with a reasonable speed, capability to zoom in/out, rotate and pan can be challenging. The work presented in this document attempts to visualize the current density and voltage distributions three dimensionally using open source tools. Chapter 3 discusses work done in the past to visualize the layout data three-dimensionally.

\subsection{An Open Source Platform for Tools Integration and Deployment}

Today's complex integrated circuit design makes use of CAD tools from different sources: commercial CAD tool vendors, in-house development groups and academic research groups. The tools from different sources will have different data formats and user interfaces. Whenever a new tool is incorporated in the design flow, there is some kind of learning curve involved, increasing the design cycle time. From a tool developers perspective tools must integrate effectively with other tools 
in the design flow in order to increase its accessibility and usefulness. Moreover each tool has its own set of integration and extensibility mechanisms. Hence tool integration requires significant investment. Integrating tools to a common platform increases its accessibility while reducing the cost of integration. From a designers perspective it provides a more consistent environment across different tools in the design flow. Also, once the tools are integrated, it is beneficial if the platform has the capability to package these tools and deploy them as a single stand alone application. Packaging should be such that minimum user intervention is required to invoke the necessary tools used in the flow.

In this work an open source platform called Eclipse is investigated. Eclipse is a java based platform that provides an integrated development, integration and deployment environment.

\subsection{Modeling IC Interconnect three dimensionally}

Figure 2.3 shows the necessary tools to visualize the current density and voltage distributions three dimensionally. First, a database or files that holds the design information such as netlist, physical layout data etc., is required. A parasitic extraction tool and a spice simulator are required to extract the parasitics from the layout and simulate the extracted data respectively. An algorithm to generate EM and IR analyses reports and a 3-D viewer capable of modeling the current density and voltage distributions three dimensionally are also required. The first step here is to visualize the interconnect three dimensionally.

The design data is usually stored in a database and exchanged through different file formats like LEF, DEF, Verilog, GDSII etc. If a 3-D viewer and the design 


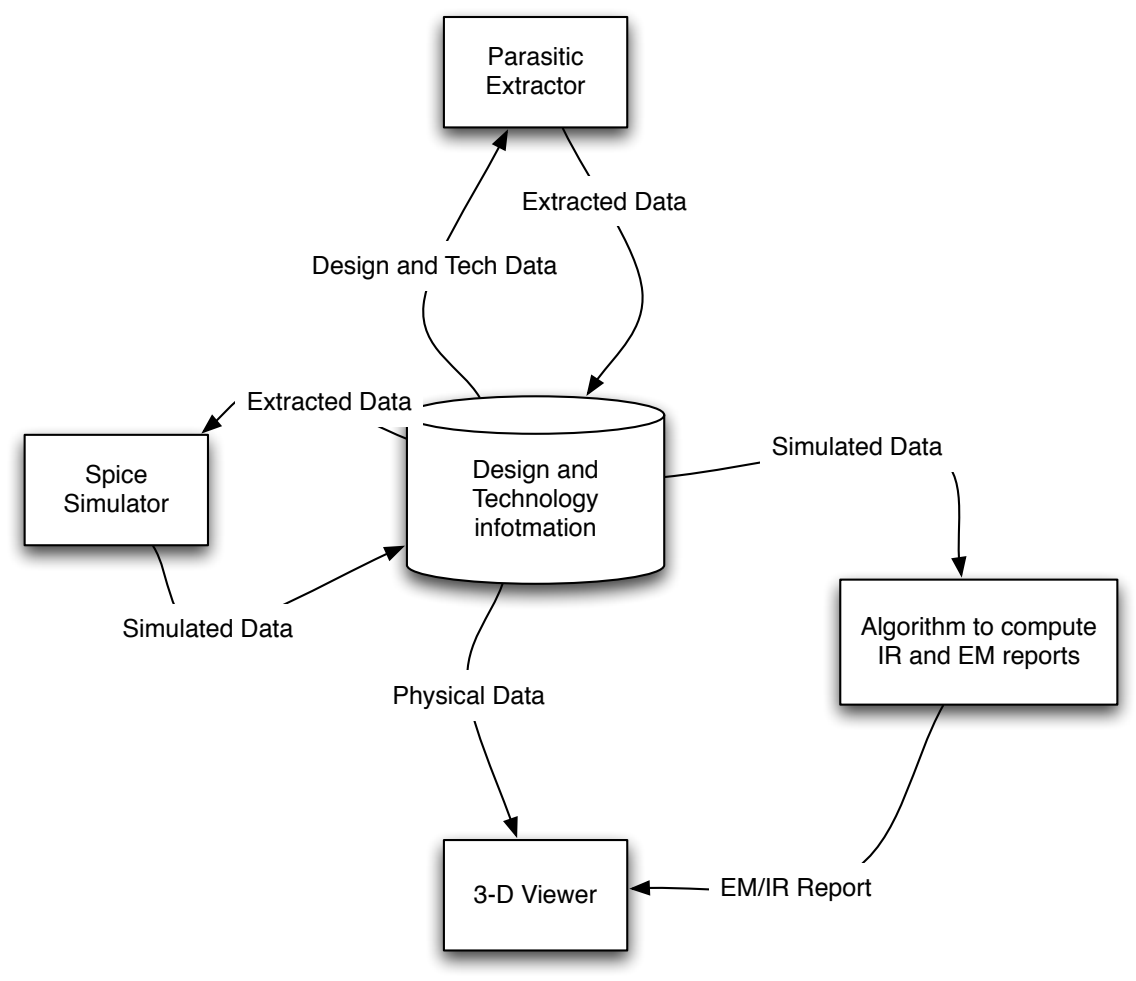

Figure 2.3: Tools necessary to visualize EM and IR analyses results three dimensionally 
data are from the same vendor, it is most likely that the 3D viewer can read the design data directly from the database or design files. If the tools are from different vendors the viewer must be extended to read foreign data formats.

In general there are different ways that an application can read data from a database. In this document the term 'Query' will be used to represent the reading of the database. Figure 2.4 shows two different ways to extend applications capability to read various kinds of data.

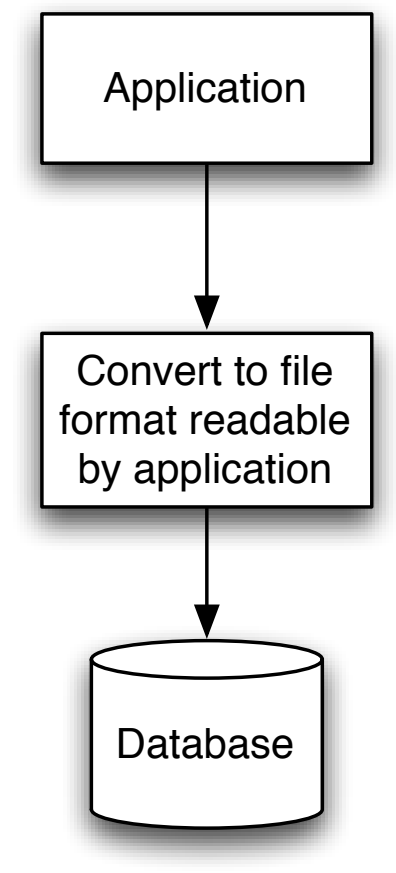

(a)

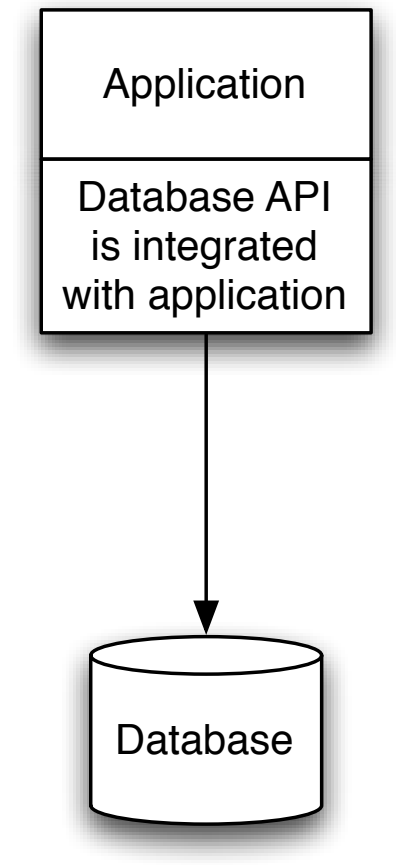

(b)

Figure 2.4: Two ways to extend applications capability to read different data formats

In case of figure 2.4(a) an intermediate conversion step is required to convert to a file format that an application can read, whereas in case of figure 2.4(b) no such intermediate step is required. This research adapts the approach shown in 
figure 2.4(a). The subsequent sections explains in detail what tools are used, why and how the adapted approach was chosen and its implementation 


\section{Chapter 3}

\section{Previous Work}

There has been some work done in the past to view the layout data 3-Dimensionally. The work presented in $[2,11,3,4]$ all discuss visualizing IC and MEMS layout three dimensionally. The tool presented by Sicard[2] visualizes the IC layout 3dimensionally. This tool uses static 3D model and it is capable of showing through a single point of view that is oblique to the circuit (Fig.3.1).

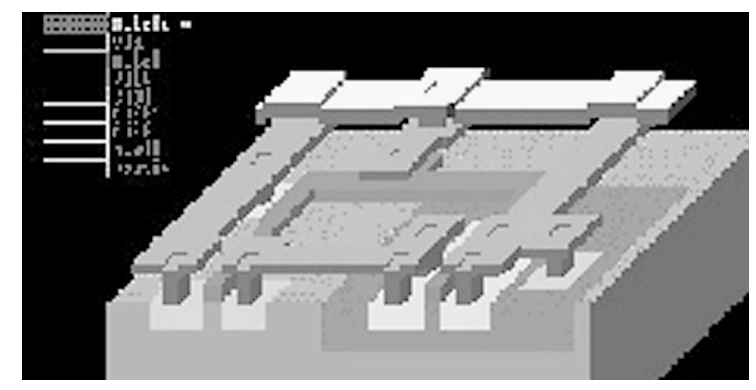

Figure 3.1: 3D IC Layout viewer by Sicard [2]

Leandro et al. [3] presents another way of viewing IC layout. It uses VRML to describe the IC layout. This tool can only read CIF file format. A tool that parses 2D layout descriptions from CIF file and converts it to 3D VRML models is also presented. The tool is called CIF2VRML and it maps CIF commands to appropriate VRML nodes/commands. The usage of VRML allows navigation 
through 3D world and multiple view points are also available. This eases the comprehension of the interconnections between layers, which defines the circuit's electrical functionality. Fig.3.2 shows 3D view of an inverter using VRML.

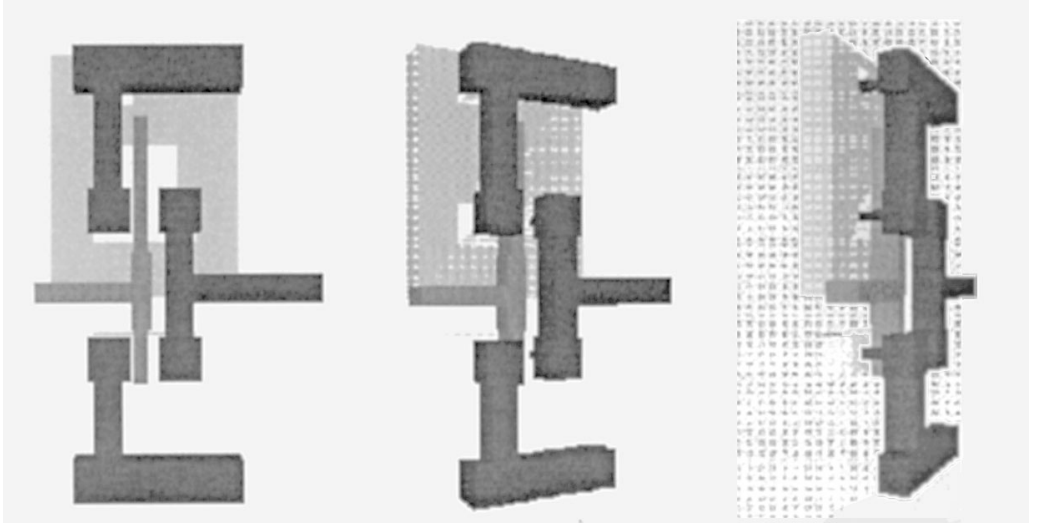

Figure 3.2: 3D view of an Inverter using VRML model[3]

The main focus of this tool is on simulating the electrical functionality and understanding how it models the circuit logic. This tool allows graphical simulations over internet. The tool was mainly built for educational purposes, to demonstrate the physical structure of integrated circuits.

The tool, Jale3D presented by Indrusiak et al.[4] is capable of editing and visualizing of ICs and MEMS layout. This tool was developed as part of the Crave Framework project. This is an extension of the work presented in [3]. Jale3D can read CIF file, and supports 2D editing. One of the main feature of this tool is that it can be loaded inside a web browser and allows 3D modeling over the WWW(World Wide Web). It uses VRML to model 3D objects and CIF2VRML to read the CIF file. The parser also maps CIF commands to Java objects. Jale3D has its own data structure to store the design. Fig.3.3, shows the data flow in Jale3D. 


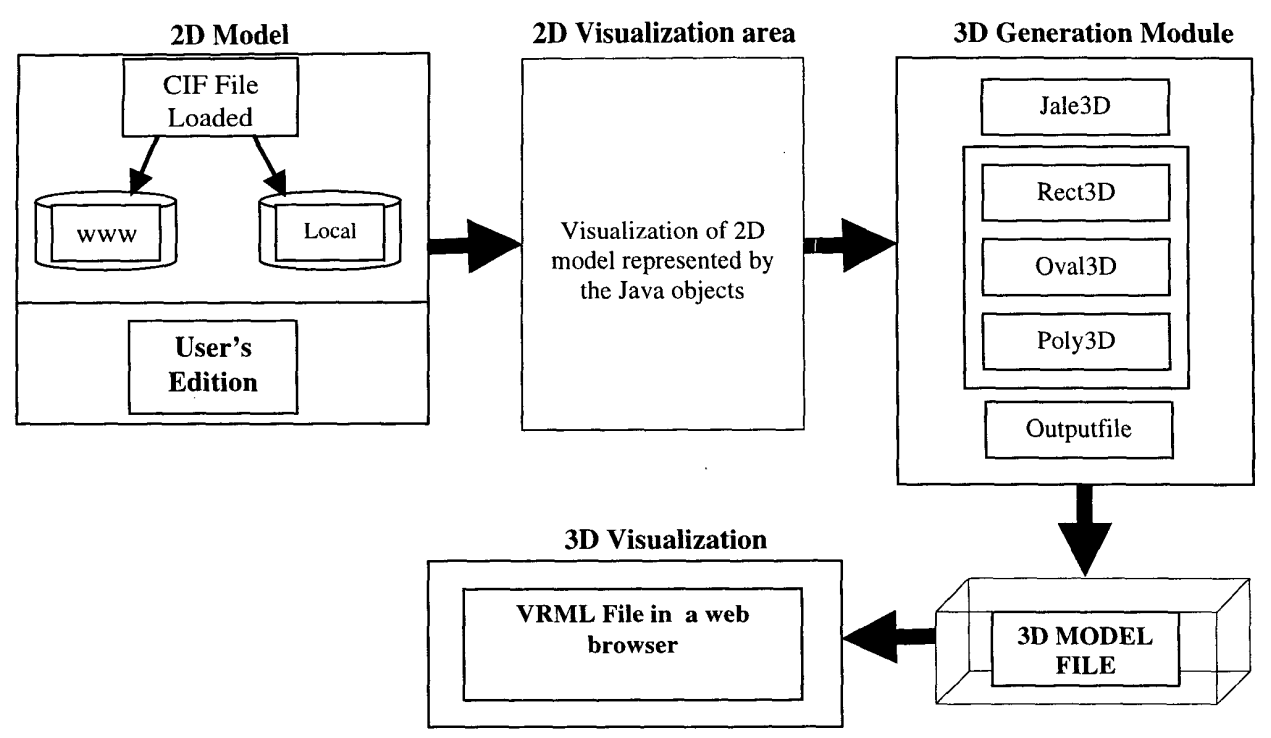

Figure 3.3: Data Flow in Jale3D[4]

The 3D generation module consists of five classes Jale3D, Rect3D, Oval3D, Poly3D and Outputfile. These classes are responsible for creating 3D objects from a 2D description and finally converting them to VRML objects.

Although the tools presented above visualize the layout 3-dimensionally, the usefulness of these tools is limited. They all support only CIF file format to read layout description, other popular formats such as LEF, DEF, GDSII etc are not supported. The CIF file format doesn't support higher-level graphical information such as texts and curves. Also information regarding the tools capability to zoom in/out, pan and rotate is not available. These features will be beneficial when dealing with large circuits. Nevertheless, it is evident from these work that there has been considerable interest amongst designers and encourages more research on the subject. 


\section{Chapter 4}

\section{OpenAccess}

The design database is the heart of any EDA tool and it is expected to perform flawlessly delivering high performance. The database needs to accommodate data from different applications [12]. More often applications have their own design database requirements. The Database must tolerate any bad data given to it. Data across each application needs to be consistent. Since most design flows incorporates different tools, communication between tools is important. In this chapter, the need for interoperability, few ways to achieve interoperability and the features of OpenAccess are presented.

\subsection{Need for Tools Interoperability}

Fig. 4.1 shows a typical Analog/Mixed signal IC design flow, along with some of the tools used in each design phase. The tools include open source tools like Magic, Electric and commercial tools from vendors like Cadence, Mentor Graphics, Synopsys etc.

Mallis [1] briefly discusses the need for interoperability amongst tools used in the design flow. A complex design for e.g, A programmable Data Converter , will have hundreds of millions transistors. The design data for such a complex 

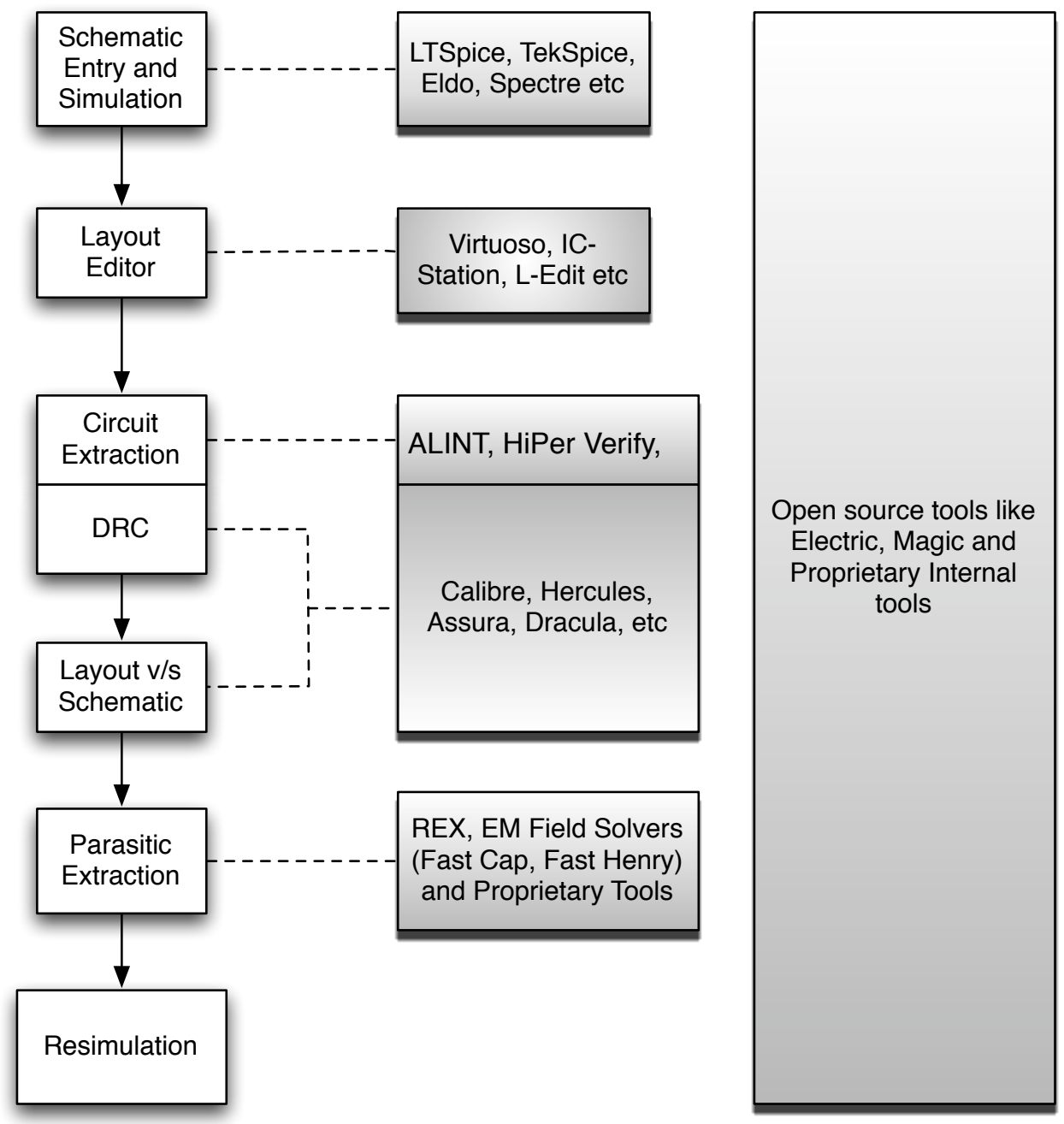

Figure 4.1: Tools used in Basic Analog/Mixed Signal Design Flow 
design can exceed a gigabyte(GB). Also the complete design is spread across tens of thousands of files. The design teams, using multiple tools, need to access these files. The data across each stage of the design flow needs to be consistent. Each tool will have a different file format which might not be readable by every other tool in the flow. For e.g, a simulation tool with schematic editor, LTSpice will have file format .asc and a layout editor, Virtuoso might have file format .tdb and a LVS tool, Calibre can only read .v files, in which case both .asc and .tdb files need to be converted to .v files. Usually scripts are used to convert from one file format to another. This is error prone and cumbersome, especially when operating on huge files containing hierarchical designs. It also adds to the design cycle time. Hence there is a need for standard file format or an API. Also the cost involved in tool integration is almost twice the cost of the application. Design exchange using standard file formats versus an API is presented in Section 4.2.

\subsection{API for Interoperability}

From the previous discussion in Section.4.1 it is clear that tool interoperability is needed to achieve better efficiency in the design flow. When using tools from different vendors, consistency in data across all tools is important. One of the popular ways of exchanging design or achieving interoperability among different tools is by using an intermediate file format. Popular intermediate file formats include Verilog, LEF, DEF and GDSII. GDSII is a binary file format, whereas others are human readable ASCII format. McGehee [13] compares design exchange using popular file formats with an API. Figure 4.2 shows a typical design exchange using LEF/DEF file format. 


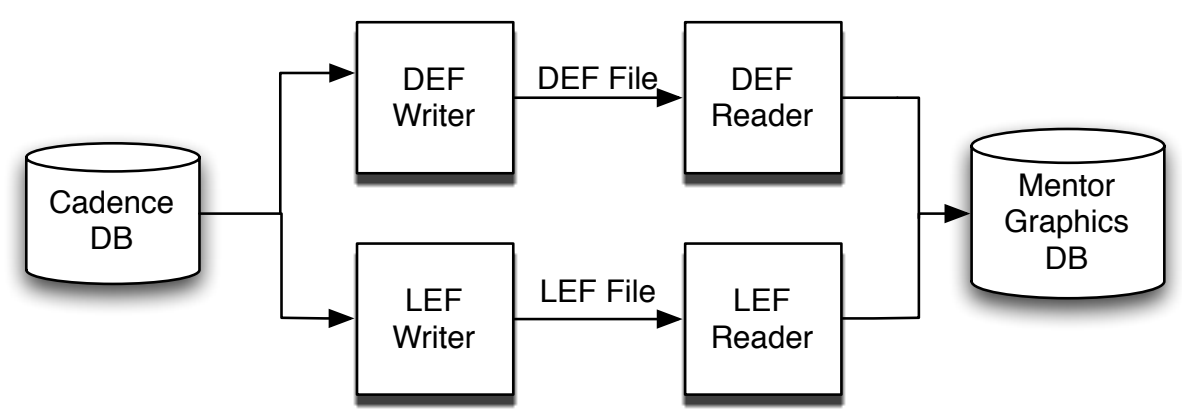

Figure 4.2: Design Exchange Using LEF and DEF

An advantage of using ASCII files to exchange designs is that they can be easily manipulated manually or by using scripts. Although file exchange seems the easy way to go about exchanging designs, it has several disadvantages. Consider the design flow shown in the figure 4.2 using LEF and DEF. Both these files are flat file formats. Since each file represents a single view of the design, multiple files are needed to transfer all the views of the design. For example, if a design contains schematic, icon and layout views, at least three files are required to transfer the design. This presents an opportunity for file skew, especially when there are different versions of the same design.

According to [14] with intermediate file format based exchange, any change in the data format comes at a cost. It impacts all the applications using it. For example, if a new data format is proposed, say GDSIII, which results in significant improvement in mask data preparation times. In order to take this advantage, tools now parsing GDSII files must be modified to parse and operate on the new GDSIII file format. Should there be another improvement, the tools must be modified again.

More often than not, the file exchange process needs additional processing. 
McGehee [13] lists some of the problems involved in transferring a design from a Milkyway (Synopsys) database to an OpenAccess database and back to Milkyway using LEF-DEF, and Milkyway to Milkyway using LEF-DEF. It is very interesting to observe that transferring designs among the same database using intermediate file format like LEF-DEF can be erroneous.

Using an API to transfer designs can help solve most of the problems mentioned above. Figure 4.3 shows an API based flow to transfer or exchange designs.

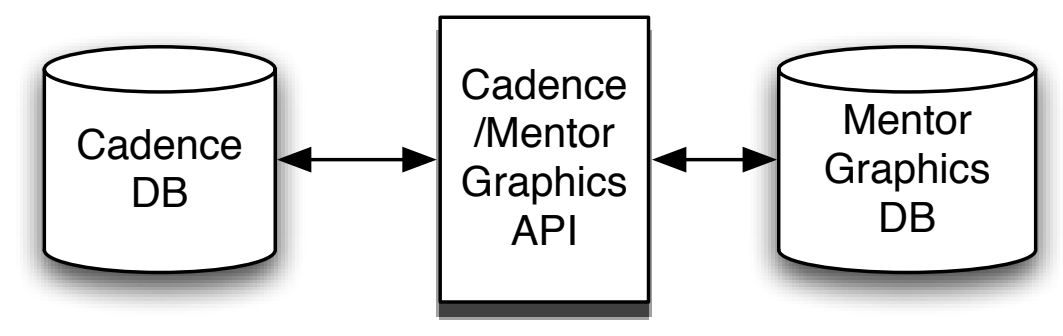

Figure 4.3: Design Exchange using an API

API flow gives the user entire control over the translation. It eliminates the need for intermediate file formats, which is the cause for most problems. A design can be shared, without converting the database. It also gives access to the entire hierarchy with all views of a design. An API is usually language based and specifies how to request information, hiding the data format. Since the applications are isolated from the data format, improvements to data representation can be made without effecting the applications. [12] Moreover the data stored in a database is very accurate and consistent. Because most databases store information in binary format, reading a database is much faster compared to parsing files. Therefore using a common API is a better way to achieve tool interoperability. 
Mallis [1] describes the previous efforts made to address the issue of interoperability. The initial initiatives were not very successful. CFI (CAD Framework Initiative) wasnt successful due to many political and business reasons at the time. CHDStd (Chip Hierarchical Design System: Technical Data) wasnt successful since it was too expensive for commercial EDA tools to refit to CHDStd and there was no publicly available database to support the specification. OpenAccess is an initiative by Si2 Inc., and major EDA tool vendors like Cadence, Synopsys, Mentor Graphics etc to provide true interoperability among tools. Semiconductor industries like HP, IBM, Intel and AMD are also contributing to the initiative. OpenAccess (OA) also provides it's reference database implementation. The reference implementation source code is open source. Section 4.3 discusses the prominent features and limitations of OpenAccess.

\subsection{OpenAccess}

OpenAccess (OA) technology is a community effort to enable true interoperability among various IC design tools. This is achieved by providing a set of standard access interface and a reference implementation which complies to that standard. The OpenAccess data model currently supports structural RTL to GDSII-level mask data. It also supports wafer related data. The database is rich enough to support Analog, Digital and Mixed Signal design data [15]. The information in the following subsections refers to the OpenAccess API documentation in addition to the works cited. 


\subsubsection{Features of OpenAccess}

As opposed to standard file formats like GDSII, OASIS, LEF, DEF, etc., OpenAccess technology provides a language based interface to request the information. This language based interface is referred to as OpenAccess API. The API is a collection of $\mathrm{C}++$ functions that operate on a hierarchical set of class definitions to request any information. The API being an object oriented, strongly typed $\mathrm{C}++$, avoids many programming errors especially ones that involve data type conversions.

One of the most prominent features of OA API is its extensibility. It includes extendable classes that permit applications to create application specific database extensions. It also allows adding attributes to the existing objects. The extensibility feature is very helpful to enhance the application to help designer analyze the problems in the design. The performance and memory requirements of such extended objects are the same as for native OA objects. This is because they work in the same way as built-in object attributes. This is a major advantage considering high performance, yet the extended objects are not publicly visible, i.e, visible only to its creator or anyone he choose to inform.

OpenAccess API represents vias as primitive database objects rather than regular instances. The work presented by Gosh [16] shows that this has provided significant improvements in Virtual Memory consumption, Design open, close and redraw times and up to 11 percent reduction in disk space. There were up to 2.5 million vias in the designs used for testing. 


\subsubsection{Features of the OpenAccess Reference Implementation}

The OpenAccess package includes a reference database implementation. This highly structured and modular database supports the OA API completely. The package also includes tests to assure the correctness of the reference database or any implementations that OA API supports. The source code of the reference database is distributed free. The OpenAccess package also includes translators for popular file formats like, LEF, DEF, Verilog, etc.

Although the OpenAccess database is implemented in $\mathrm{C}++, \mathrm{Tcl}$ and Python bindings are also available. OA package includes Tcl bindings, and LSI Corp. provides Python bindings. These bindings are also open source. Scripting languages are very useful in generating analyses reports. However neither Tcl nor Python bindings support the complete OA API. For example, application defined extensions, observers etc., are not supported.

The reference implementation provides direct and incremental access to the information. For example, if an application requests certain pin on a specific net, the database will return only that information. When performing such an operation, the reference database doesn't load the entire set of data. This way overall performance is greatly improved, especially when compared to parsing a sequential file. Similarly, incremental writing to the database is also possible. The OpenAccess reference implementation was built from the start to provide a production quality, high performance database for the design community and to reduce the barrier of entry to OpenAccess.

Considering the features discussed above, this research utilizes the design stored in the OpenAccess reference implementation and its API to model IC interconnect 
three dimensionally.

\subsubsection{OpenAccess Organization}

The OpenAccess API is organized as set of packages. Each one of these packages provide a part of the total functionality. There is a top level header file for each package. It contains functions and classes to access the required information. Implementation of these functions and classes are distributed as a shared library for each package.

OpenAccess has a total of six packages. Three database packages and three more additional general purpose packages. Design, Technology and Wafer are the database packages. Design Management, Base and Plug-in are the additional packages. Figure 4.4 shows the information model of these six packages and the API. The following is a brief description of these packages.

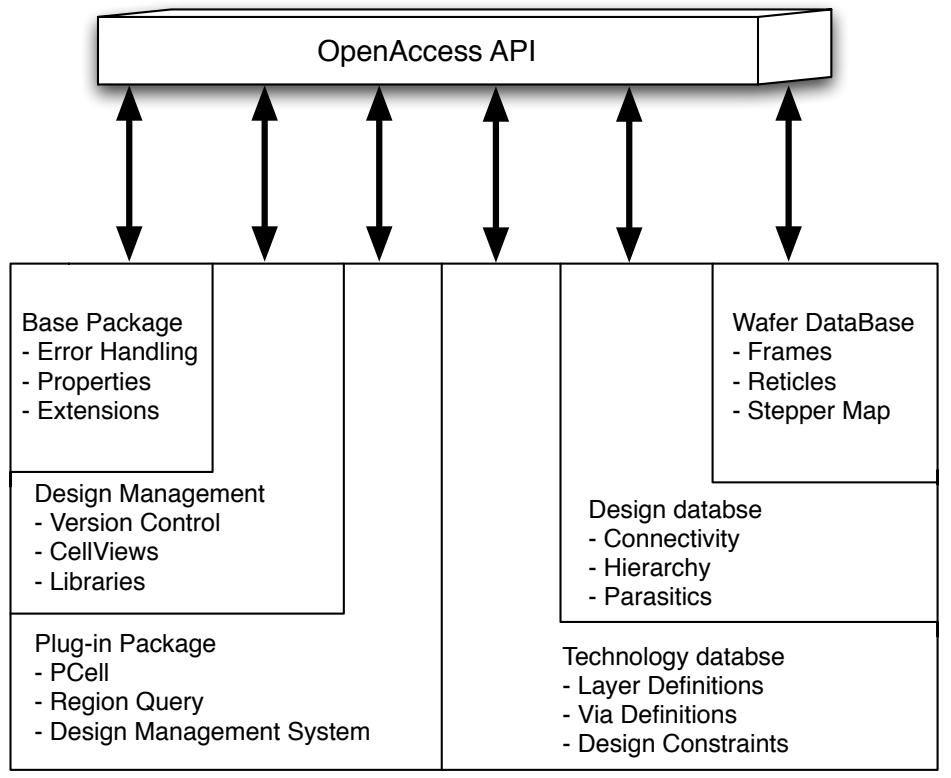

Figure 4.4: OpenAccess - API Packages 
The API for the Design Management package(DM) consists of functions necessary to keep the design libraries, cells and views organized. Other features like synchronization, version control etc., are handled through the DM system. The DM systems are built on OpenAccess Plug-in infrastructure and are loaded during run time. OpenAccess provides two DM systems, FileSys DM system and Turbo DM system. The FileSys DM systems organizes the design files and library information using file systems directory structure where as the Turbo DM system is a server based system. The Base package provides common functions like error handling, namespaces, strings, extensions etc. OpenAccess allows run time customization of certain functions. The Plug-in package contains a set of specific API interface classes to enable dynamic loading of third party software to integrate with OA run time model and manage certain design data like DM Systems, Version Control Systems etc.

Design, Technology and Wafer databases have their own data model. A Data Model describes how the objects are stored in the database, their relationships with other objects and their proper usage by the applications. The wafer database holds the manufacturing information like layout of reticles for each layer, frames etc. This is particularly helpful in yield analysis. Since this research focuses on reliability analyses of circuits, discussion of the wafer database is out of the scope of this document. Design and Technology databases are presented next.

\section{Design Database}

As the name specifies, the Design database manages everything related to a design such as its connectivity, hierarchy, layout, floor planning, parasitic information etc. The oaDesign class is the main class associated with the Design Database. A 
design's cell views like schematics, netlist, layout etc., are stored as oaDesign in OpenAccess. OpenAccess organizes each design as a Design Library, Cell and Cell View as shown in Fig.4.5. Each oaDesign in a cell view can be accessed by specifying a particular library, cell and view names. A cell view is persistently stored as a single file or multiple files, e.g, schematics may be stored as schematic.oa. Managed classes in the design database package derive from the oaDesignObject class. Managed classes are discussed in more detail in section 6.1.1

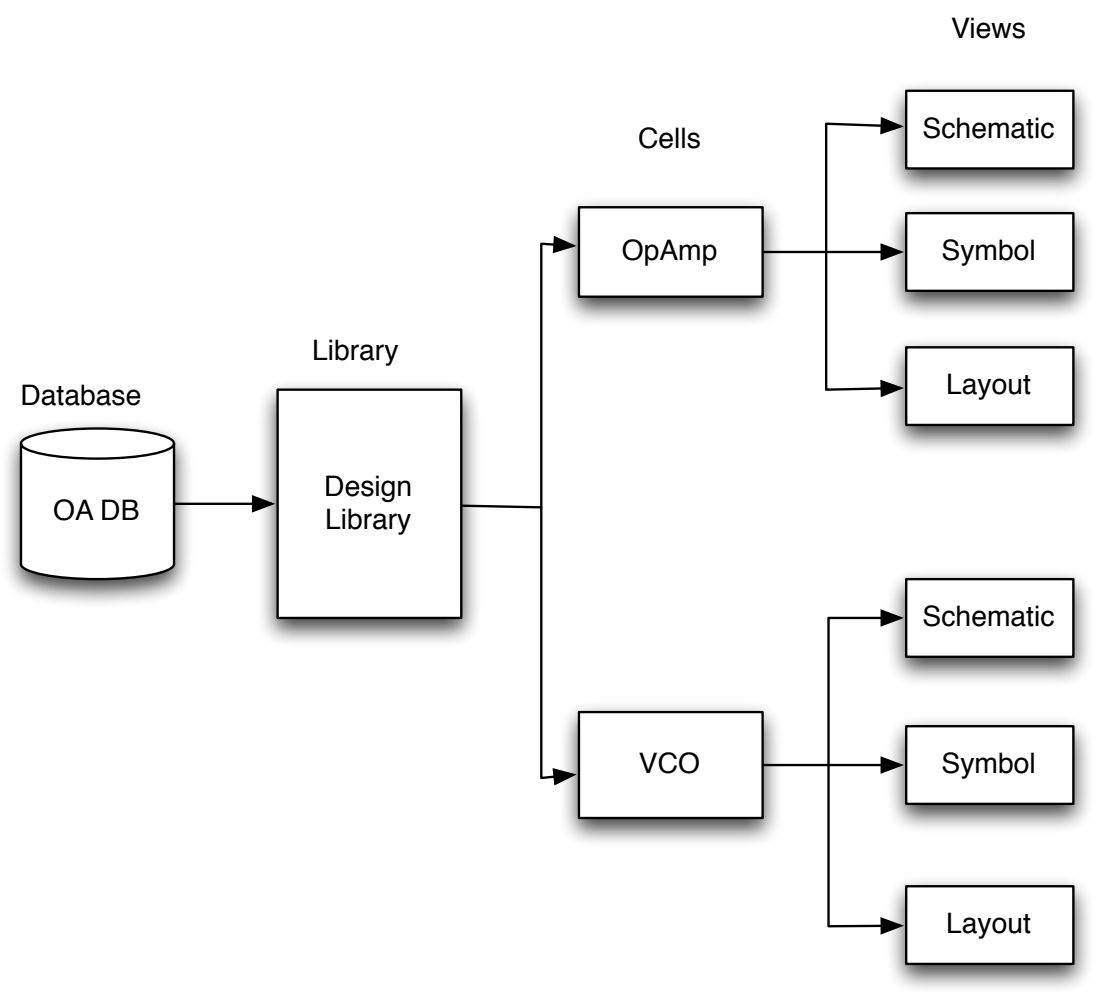

Figure 4.5: OpenAccess - Design data organization 


\section{Technology Database}

The technology database manages the technology data like via and layer definitions, routing and foundry constraints etc. The technology data may not be limited to a particular design but across all the designs made from a given technology. The oaTech class is the main class associated with Technology Database and managed classes are derived from oaTechObject class

Figures 4.6 and 4.7 shows the schema diagrams of oaDesign and oaTech classes respectively. These diagrams shows the high-level relationship of the primary classes in their category. For example, figure 4.6 shows the relationship of the oaDesign class with other important classes like oaTech, oaLib etc. Note that these diagrams are at information model level and doesn't represent the complete API. The legend for these diagrams is shown in the figure $\mathrm{E}$

\subsubsection{Limitations of OpenAccess}

The features discussed previously have influenced popular CAD/EDA tool vendors such as Synopsys, Mentor Graphics, Cadence etc., to support the OpenAccess API. Technology providers like TSMC, MOSIS etc., are also distributing process design kits (PDKs) as OA database. However during the course of the work, a few limitations of OpenAccess were observed and are presented below:

- It could be a significant investment to convert an established tool to run natively on OpenAccess.

- Although OA data model supports parasitic elements, primitive circuit elements such as resistors, capacitors, inductors, transistors etc., are not defined in the OA data model. 


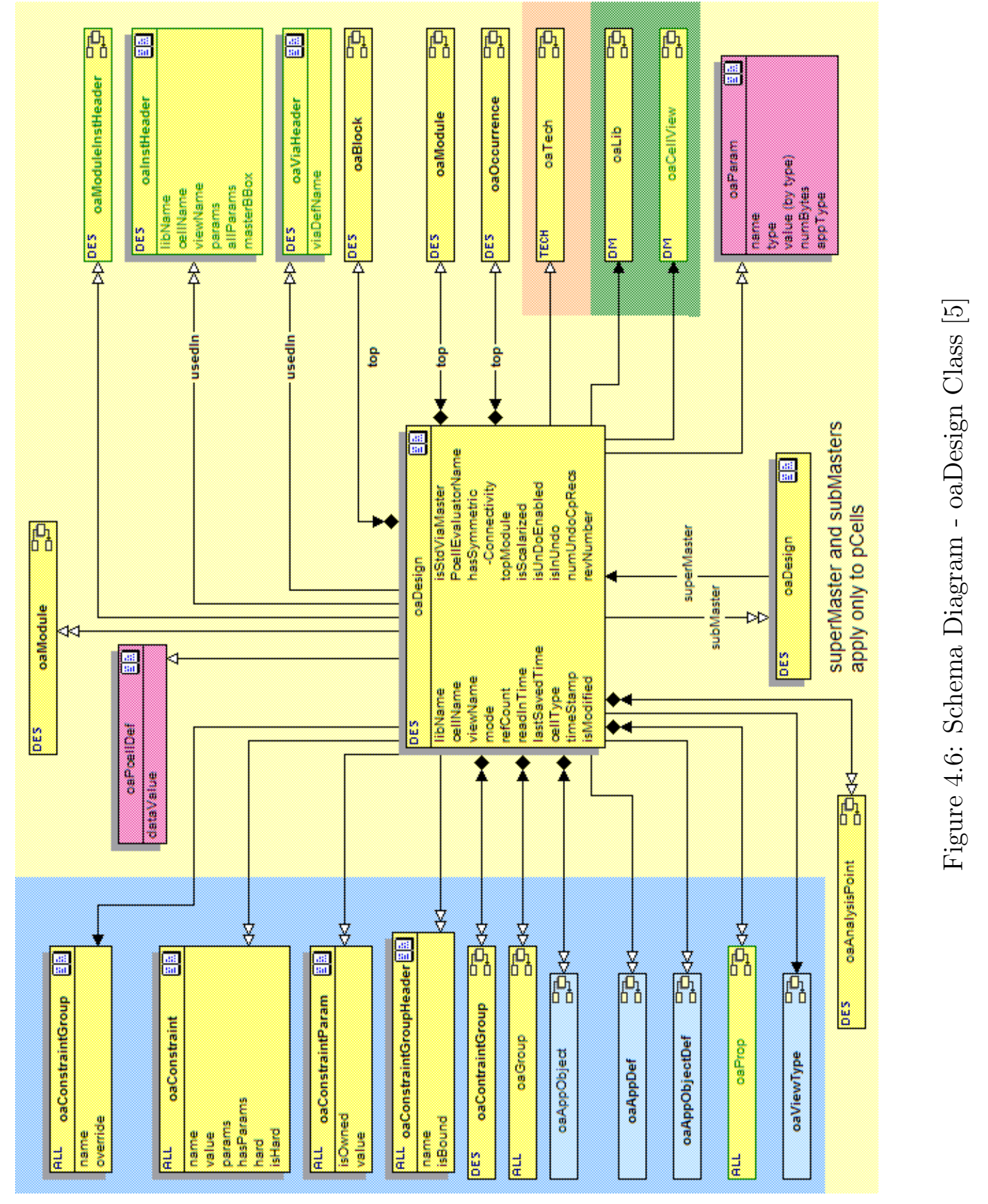




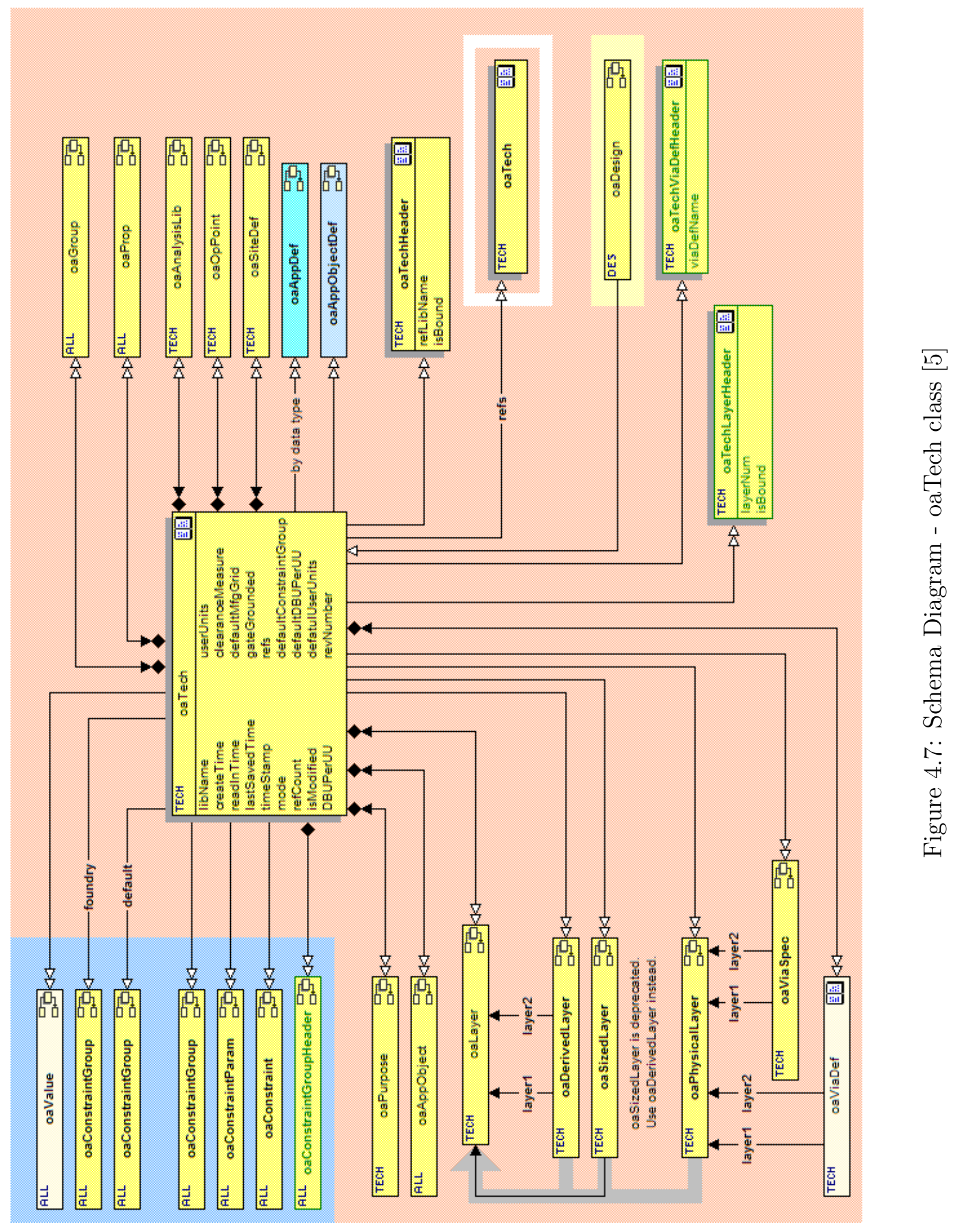


- The technology database doesn't support transistor model information such as threshold voltage, gate oxide thickness, mobility etc. Therefore the technology provider has to supply the transistor model information in a different file.

- Spice data is currently not supported in the OpenAccess data model. Hence integrating spice tools with OpenAccess can be arduous.

- Although OpenAccess reference implementation is open source, its license is more restrictive compared General Public License (GPL).

- Although it is free membership is required to use OpenAccess. Non-Member can only use it for evaluation. More over, the latest distributions are only available to the coalition members only, which is a paid membership. 


\section{Chapter 5}

\section{Integration of $3 \mathrm{D}$ viewer with OpenAccess}

Viewing IC interconnect three dimensionally requires a 3D viewer or renderer application. The article presented in [17] compares different 3D tools available. Only Art of Illusion (AOI) and Blender are distributed under the open source general public license. Both these tools have a similar feature set, but the user interface of Art of Illusion is a lot more intuitive than Blender. This is very important, especially to learn how a tool models different shapes. Hence Art of Illusion was chosen for this work. In this section some of the features of AOI and integrating it with OpenAccess are presented.

\subsection{Art of Illusion}

Art of Illusion is an open source, 3D modeling and rendering tool. It is entirely written in Java, therefore any platform using the Java Virtual Machine should be able to run this software. This application was the Source forge project of the month for April, 2007. Art of Illusion supports uniform and layered textures and materials to represent internal properties of objects. These features can be used to

model current density and voltage distributions. Since this tool was not designed to be integrated in to the Integrated Circuits design flow, representing all the 
objects related to the circuit design such as resistors, capacitors transistors etc., is difficult. But the main focus of this research is on layout data, which consists of basic geometric shapes like, rectangles, polygons etc. Since Art of Illusion can model these shapes, modeling interconnect three dimensionally is possible using this tool. Moreover there are no open source 3D viewers that readily supports IC design data.

\subsection{Integration of Art of Illusion with OpenAccess}

Since both Art of Illusion and OpenAccess are from different sources, they work on different data formats. These tools must be extended so that they work together. The tools have a different set of extensibility mechanisms. In this section, different ways to extend Art of Illusion with OpenAccess are discussed. Section 5.2.1 presents extending AOI so that it can read the OA database. In section 5.2.2 different ways an application can integrate with OpenAccess are illustrated.

\subsubsection{Extending Art of Illusion}

To model IC interconnect, Art of Illusion needs to read the design data from the OA database. In order to do this Art of Illusion needs to be integrated with OpenAccess. With AOI being a java application and the OA API written in $\mathrm{C}++$, integration is challenging. From Art of Illusion's perspective, it can extend its capability to read foreign file formats through plugins that extends the artofillusion. Translator interface, Fig. 5.1. Again this plugin is in java and has to query the $\mathrm{C}++$ database. A workaround for this is to create a Java wrapper for $\mathrm{C}++$ API using Java Native Interface (JNI). However implementing JNI for a huge database 
like OpenAccess is very complicated. Section 5.2.3 discusses more on JNI, an automation tool called SWIG and their drawbacks.

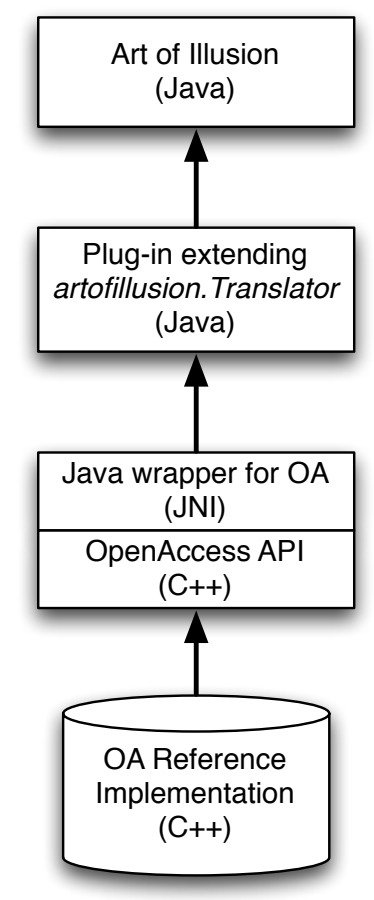

Figure 5.1: Extending Art of Illusion functionality

\subsubsection{Application Integration with OpenAccess}

There are various ways an application can be integrated with the OA database. The application under consideration here is Art of Illusion. The figure 5.2 shows three ways an application can integrate with OpenAccess.

- File Exchange - In this case, a translator is used to convert data in the OA database to the data format which the application reads. The translator uses the OA API for the conversion. 


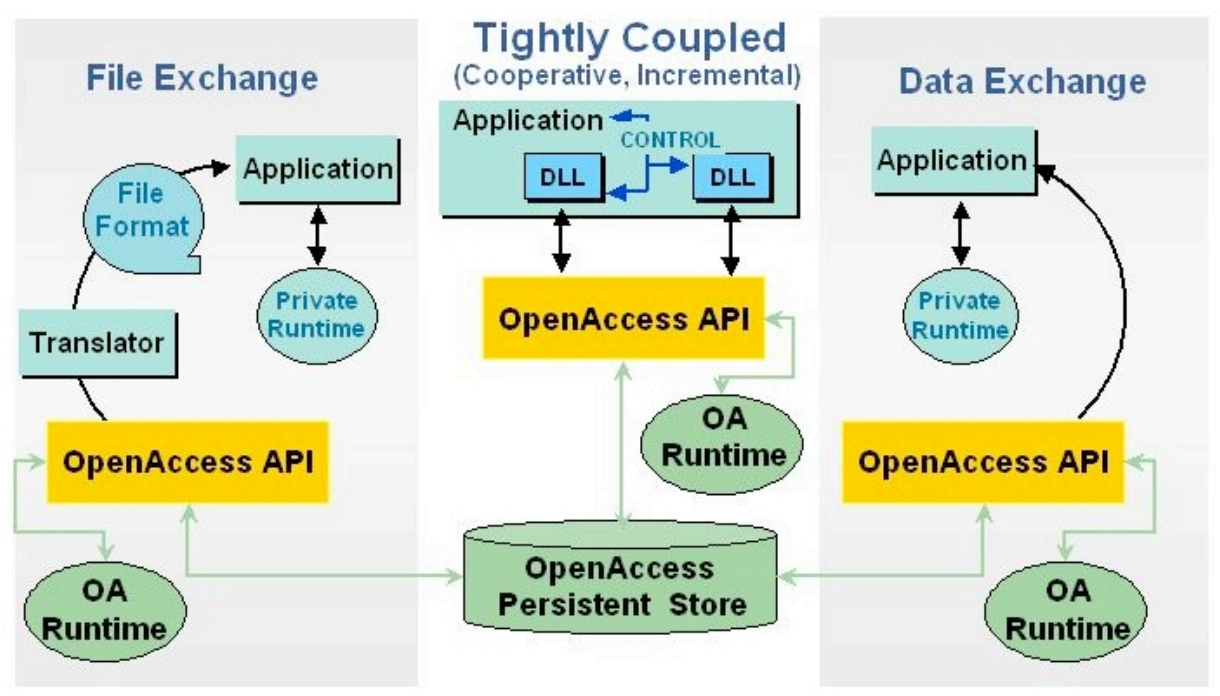

Figure 5.2: Application Integration with OpenAccess

$[5]$

- Data Mapping - The Application reads the data from the database using OA API and converts it into a different runtime model which the application uses.

- Tight Coupling - The application shares the OA runtime model and no conversion is required

Integration using Data Mapping and Tight coupling techniques require creating Java wrappers for the C++ API using JNI. Again section 5.2.3 discusses the implications of using JNI. In File Exchange, a $\mathrm{C}++$ program can read the data using OA API and convert it to a file format that Art of Illusion can read. In this case the $\mathrm{C}++$ program acts as a translator. The work presented here uses the File Exchange approach. Since Art of Illusion supports importing .OBJ file formats natively, the design data is written in to .OBJ format. Moreover most 3D modeling tools can read .OBJ file format, hence the designer can use his/her 
favorite $3 \mathrm{D}$ tool to view the physical design data.

\subsubsection{Java Native Interface and SWIG}

The Java Native Interface is a programming interface that allows Java programs to call native libraries written in $\mathrm{C}$ or $\mathrm{C}++$. Since the OpenAccess API is huge, implementing JNI for such an API is very complicated. Table 5.1 shows the number of classes, functions etc., in the API. In fact these numbers can be much more, since the data shown in the table is from the API release in the year 2004. Since then OA has updated from Data Model 3 to Data Model 4. Data Model 4 adds support to $45 \mathrm{~nm}$ process by defining additional constraints. Which means some more classes and functions were added to the API. Although this work deals with only Design and Technology database APIs, the number of classes and functions to implement in JNI is still large and requires automation.

\begin{tabular}{|c|c|}
\hline Feature & Count \\
\hline Classes & 1955 \\
\hline Enum Types & 170 \\
\hline Public Member Functions & 16275 \\
\hline Static Member Functions & 8297 \\
\hline
\end{tabular}

Table 5.1: Overview of OpenAccess API Functions and Classes [18]

The Simplified Wrapper and Interface Generator (SWIG) is an open source tool that simplifies interfacing other languages with $\mathrm{C}$ and $\mathrm{C}++$ programs. It supports languages like, Perl, Tcl, Python, Ruby, Java etc. The main purpose of SWIG was to ease the integration of scripting languages with $\mathrm{C}$ programs. Later on it was extended to add support for $\mathrm{C}++$ language. However SWIG doesn't support all the features of $\mathrm{C}++$. For example, certain operator overloading functions 
(new, delete), nested classes etc., are not supported. Nested classes and operator overloading functions are extensively used in OpenAccess API. More over wrapping $\mathrm{C}++$ templates is a struggle, because manual editing of the header file is required. Considering the number of OA API classes, even with SWIG a lot of manual editing is required.

More over there are few drawbacks in with the JNI itself, like:

- An application relying on JNI looses the platform independence capability that Java offers. In this case Art of Illusion will no longer be platform independent.

- The JNI framework doesn't provide automatic garbage collection and the native $\mathrm{C}++$ code must assume this responsibility

- Passing an invalid argument like, NULL to a JNI function, can result in undefined behavior, most likely resulting in the virtual machine crash. These kind of errors are not detected during the compile time. Hence it is very difficult to reproduce and debug such errors.

After evaluating the drawbacks of SWIG, JNI and the time complexity involved to create Java wrappers for the OpenAccess API, file exchange technique is implemented in this work. Moreover since the main function of the application here is reading the design data, using the File Exchange technique preserves the data integrity in the database. 


\section{Chapter 6}

\section{Query Implementation}

Implementing a query to get the geometric data of interconnect requires an indepth understanding of how the design information is stored in the OpenAccess database along with the knowledge of the necessary API classes. In this chapter a classification of OpenAccess API classes, the query algorithm and the query implementation are presented. Again all the information regarding the API classes refers to the OA API documentation [5].

\subsection{OpenAccess API Classes}

OpenAccess API consists of classes, initialization functions, operators, library utilities and constants. Since classes represent data objects, their relationship with the design and majority of the interface, only the API classes are discussed in this section. The API classes are classified into 3 major groups:

\section{- Managed Classes}

\section{- Utility Classes}

\section{- Interface Classes}

Functions of each of these classes are enumerated next. 


\subsubsection{Managed Classes}

A class that has data persistently stored in an OpenAccess database is called a Managed Class. These type of classes collect and manage various kinds of data and metadata related to a design, other information such as export formats, for example, LEF and DEF and tool specific information. The oaObject class is the abstract base class for all managed classes. Managed classes are categorized as Databases, Extensions and Constraints.

- Databases - These classes are responsible for managing the objects that maps to a persistent disk representation requiring individual granularity of managed control and locking. For example, oaDMObject class provides functions to control different versions of the design.

- Extensions - Most of the time applications define their own object types to hold proprietary information related to the design that is not common across different tools. These objects are not standardized in the API. Extension classes are usually used for such application defined data. For example, oaProp class is the abstract class for all types of properties including application-defined. Properties are simply name/value pair.

- Constraints - These classes are used to model rules for design and manufacturing processes. For example, the oaConstraint class is the abstract class for all constraints. A DRC tool can use these constraints. Note that this is not limited only for design rule checking, an application can use it for other purpose as well.

Managed classes and their classification is shown pictorially in Fig.6.1. 


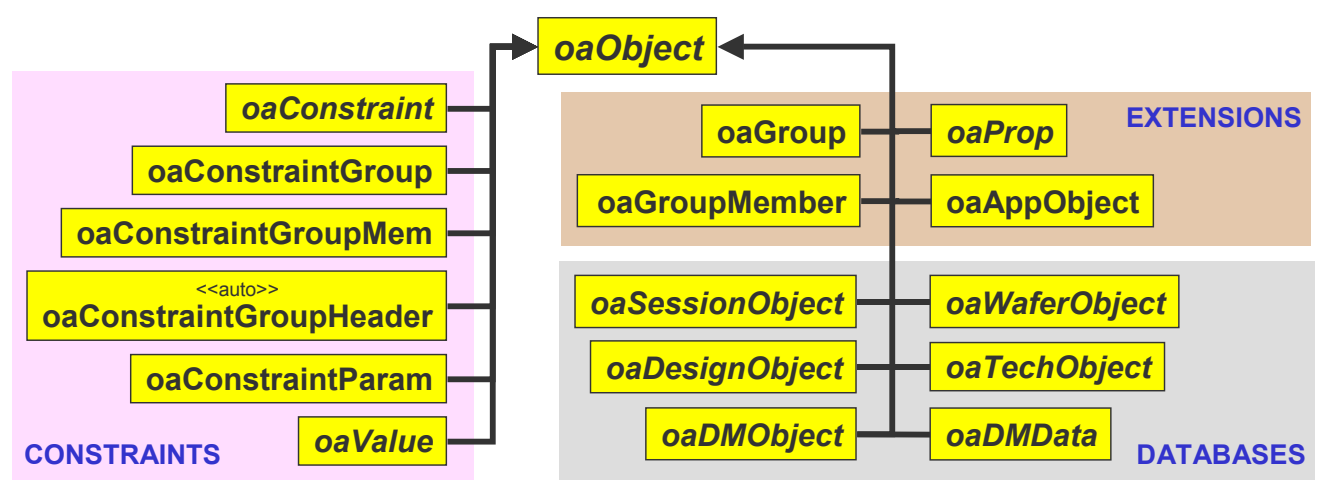

Figure 6.1: Managed Classes[1]

\subsubsection{Utility Classes}

Utility classes are usually used to access or change a managed object's attributes. Their primary usage is to pass these values between the API and the application. The application owns objects created by utility classes and their storage is also managed by the application. The table 6.1 shows some of the Utility classes and an example use with managed objects.

\begin{tabular}{|l|l|}
\hline Utility Class & Example Use with Managed Object \\
\hline \hline oaName & Set or Get the name on or from a net (oaNet) \\
\hline oaTransform & Set or Get the placement information on or from an instance (oaInst) \\
\hline oaPointArray & Set or Get the list of points comprising a polygon (oaPolygon) \\
\hline
\end{tabular}

Table 6.1: Utility classes and examples[1]

\subsubsection{Interface Classes}

OpenAccess supports plugins to customize some of its functions at the run time. The interface classes allow dynamic loading of plug-ins and integrate with the managed design data through some of the API classes. The table 6.2 shows some 
of the Interface classes along with the OA classes it can integrate with.

\begin{tabular}{|l|l|}
\hline Interface & OA Class Integration \\
\hline \hline IEvalText & oaEvalTextLink, oaEvalText \\
\hline IText & oaTextLink \\
\hline IPCell, IPcellGen, IPcellDefMgr & oaPcellLink, oaPcellDef \\
\hline RegionQuery & oaRegionQuery \\
\hline
\end{tabular}

Table 6.2: Interface Classes and OA Class Integration[1]

Most of the design and technology information are created and managed by the Managed classes and the attributes of these objects are set by the Utility classes. Since this work deals mainly with layout and technology information, Managed and Utility classes are of great importance. The following example shows the usage of managed and utility classes.

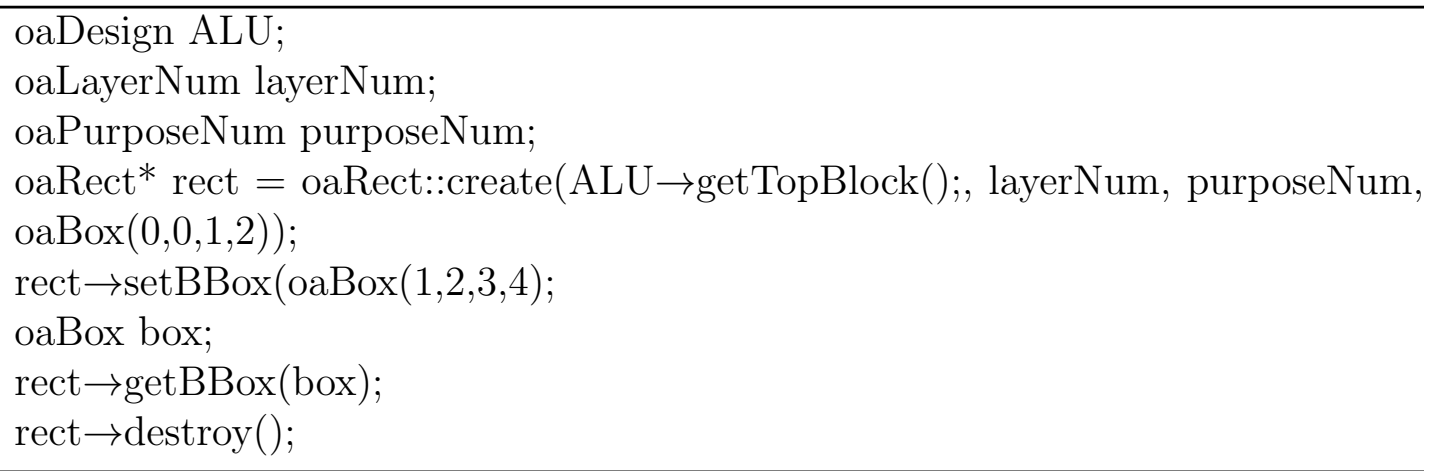

In the above example oaRect class creates a rectangle, 'rect' object of $(0,0,1,2)$ with a particular layer number (layerNum) and purpose number (purposeNum) in the top block of ALU design. Since oaRect class is a Managed class, 'rect' is a managed object and the API manages its relationship with the design. The setBBox () and getBBox () are the utility classes used to set/modify and get the box attribute of the object rect respectively. 


\subsection{Query Algorithm}

Since interconnect is a subset of a layout, the layout view of the cell is of main interest. The layout view basically contains information of different layers (poly, metal1 etc) for different purposes (text, drawing etc) having different shapes(rectangle, polygon etc), of the cell. This is pictorially represented in the figure 6.2. To visualize IC interconnect, geometric coordinates of different shapes needs to be queried from the OA database.

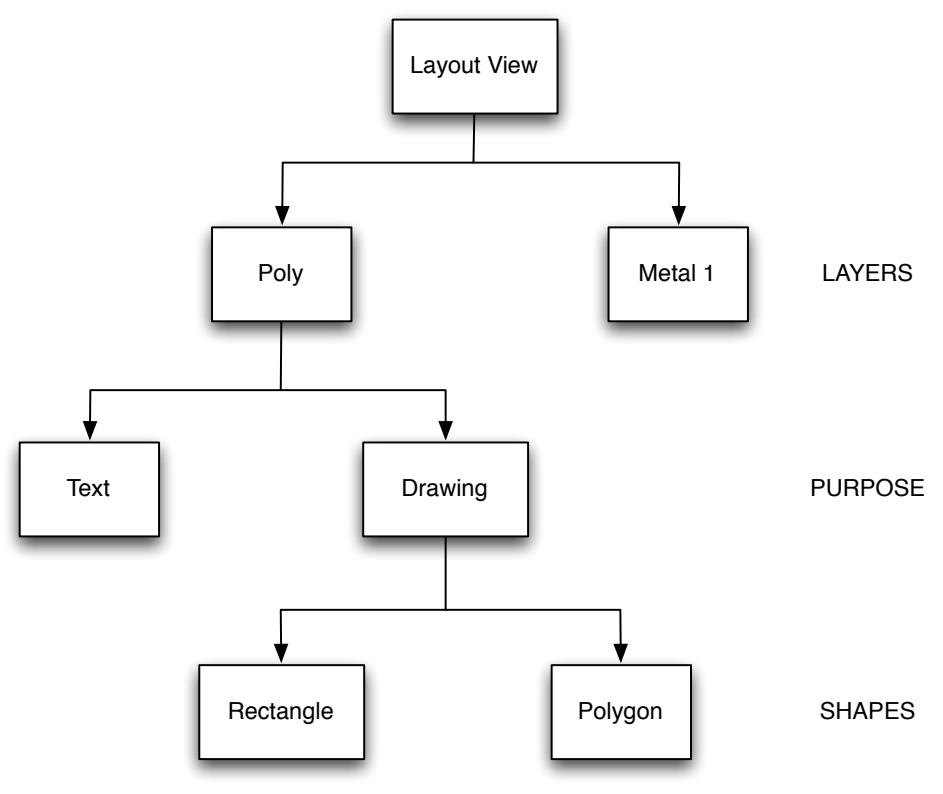

Figure 6.2: Layer-Purpose-Shape Relationship

The interconnect in the layout include different metal layers like poly, metal1 etc., contacts and Vias. These are basically geometric shapes, having different set of attributes and electrical properties. OpenAccess database supports different shapes associated with interconnect layers. A Layer Purpose Pair Header (LPPHeader) is automatically created by the database whenever a shape with a 
particular combination of layer and purpose number is created. If a shape with same layer and purpose number is created it shares the same LPPHeader. For example, in the figure 6.2 both Rectangle and Polygon will share the same layer purpose pair number.

To visualize the physical data, the coordinates of all the required shapes associated with each layer need to be queried. OA provides various API calls to implement such a query. One of the ways to implement the query is by iterating through each LPP Header in the layout and querying the coordinates of all the shapes associated with each LPP Header.

The flow chart in the figure 6.3 shows an algorithm to implement a query to get the geometric coordinates of all the shapes in the layout. The algorithm can be divided into two steps LPP Query and Shape Query. Each query along with the classes used to implement the query is discussed in sections 6.2.1 and 6.2.2.

\subsubsection{LPP Query}

In this section, an algorithm to query the layer purpose pair (LPP) numbers in the design is presented. This includes checking if the layer is an interconnect layer and if the purpose of the layer is of interest, i.e., drawing. For example, a 'nwell' layer with a purpose 'fill' is not of importance and hence it is ignored.

To implement the LPP query, the collection of all Layer Purpose Pairs, represented as oaLPPHeader objects, in the design needs to be queried. The oaLayerHeader class provides indirect access to the collection of oaLPPHeader objects via oaCollection <oaLayerHeader, oaLPPHeader $>$ ::getLPPHeaders() function. The oaCollection can be implemented as oalter class, which can be used to iterate over the objects in oaCollection. Then oalter::getNext() function will return the next 


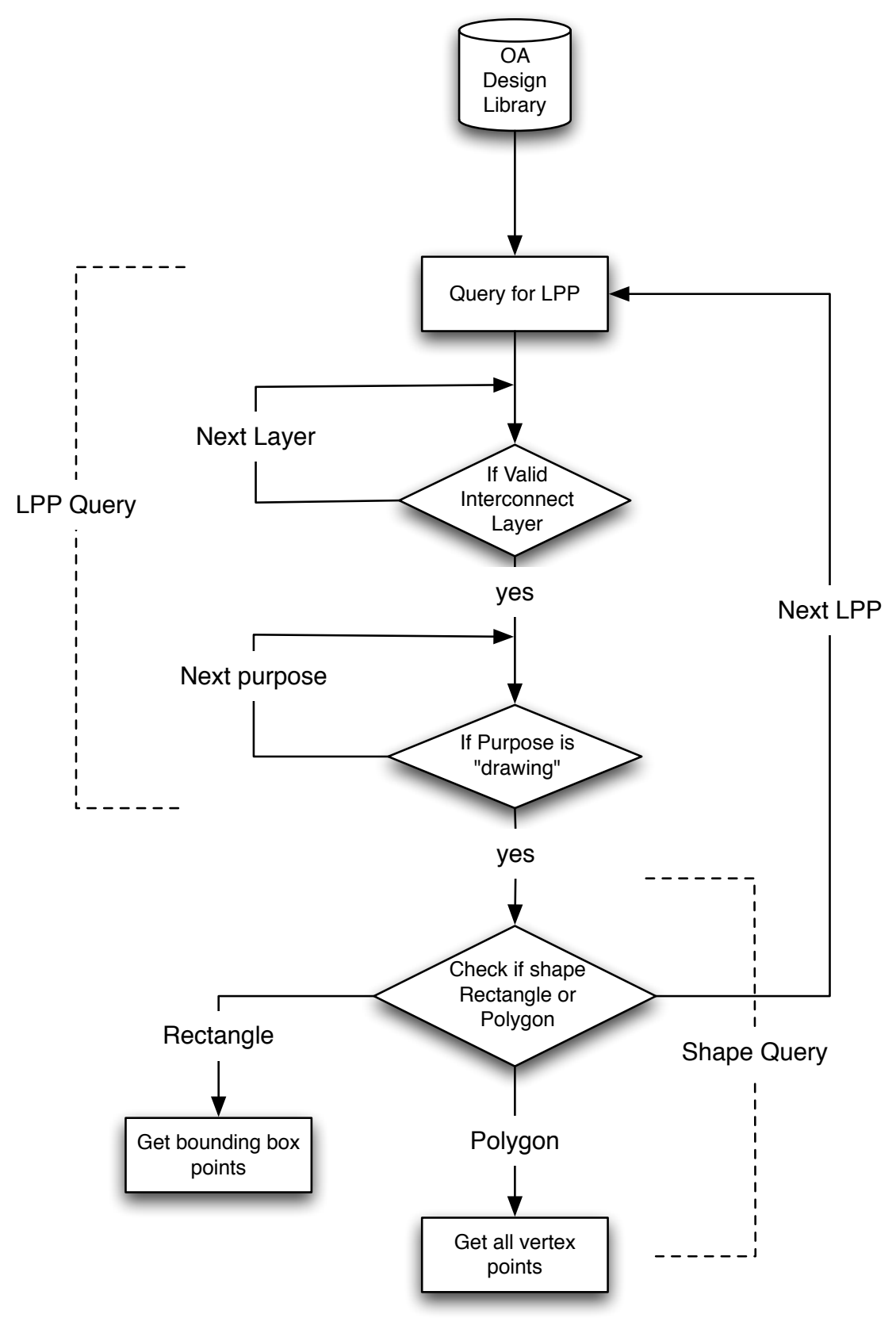

Figure 6.3: Query Algorithm 
object in the collection.

To get the layer and purpose numbers of the LPPheader object, following two functions can be used:

oaLayerNum oaLPPHeader::getLayerNum()

and oaPuposeNum oaLPPHeader::getPurposeNum()

The layer and purpose names are usually mapped in corresponding technology database and following functions can be used to get layer and purpose names oaLayer::find(oaTech*, oaLayerNum) oaPurpose::find(oaTech*, oaPurposeNum)

Once the layer and purpose information are obtained, they can be validated with a predefined set of valid layers and purpose. In this work valid layers are poly, contact, metal1, via1 and metal2 and the valid purpose is 'drawing'. For a valid layer purpose pair (LPP), all the shapes associated with the LPP and their geometric coordinates are queried. This is presented next as Shape query.

\subsubsection{Shape Query}

Querying the geometric coordinates of all the shapes associated with a valid LPP is discussed in this section. OpenAccess supports different shapes having different set of attributes. A rectangle will have a bounding box that forms the rectangle, a polygon will have an array of points in a bounding box. All shapes are created using one of the subclasses of oaShape base class. Figure 6.4 shows the information model of the oaShape class. The figure also shows the attributes associated with each shape.

The oaLPPHeader::getShapes() function returns a collection of shapes in a given LPP Header. Similar to the LPP query, the oalter class can be used to iterate over 


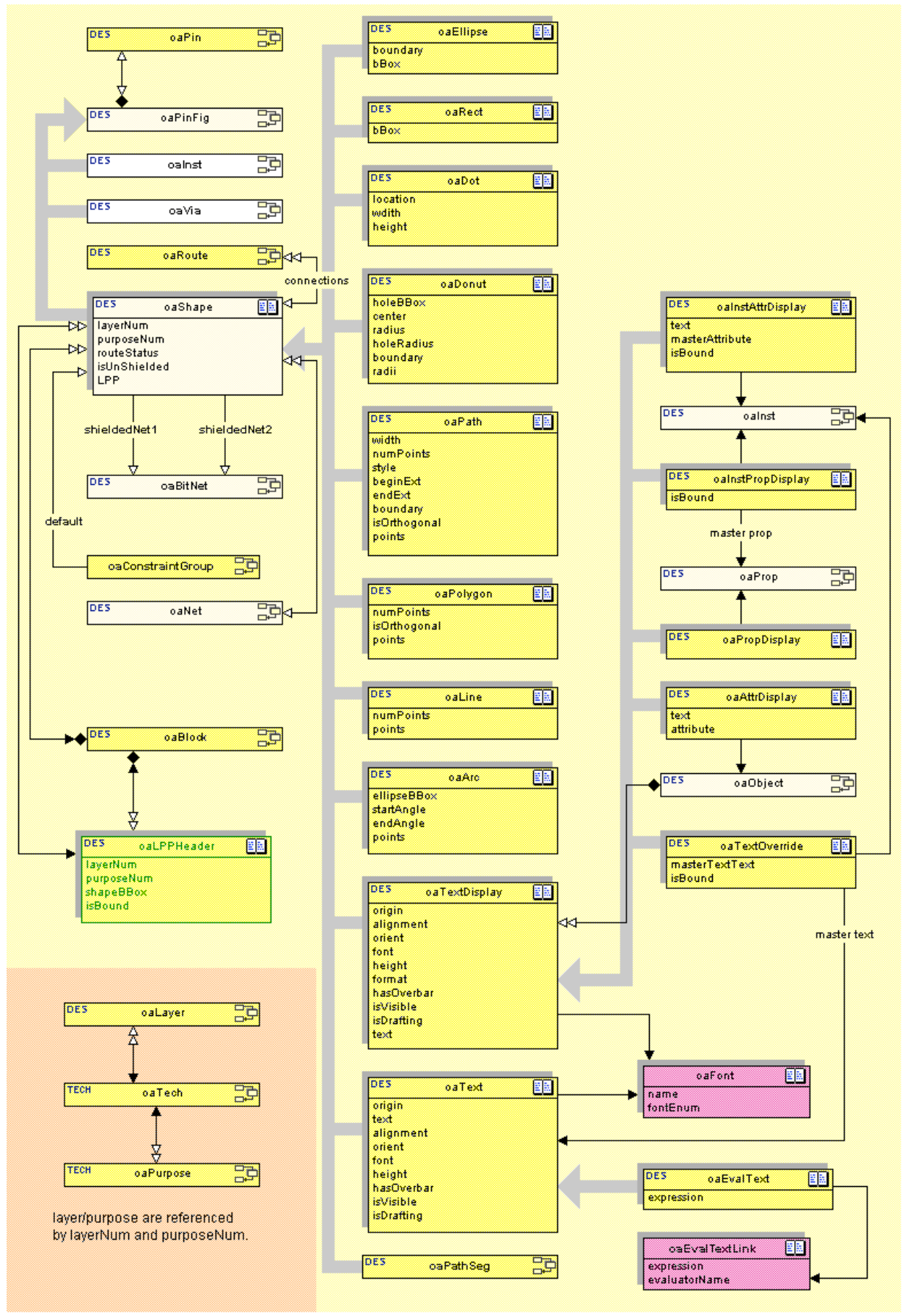

Figure 6.4: Information Model of oaShape class

$[5]$ 
all the shapes. Shapes can be of different type. The oaShape.getType () function returns the type of the object as oacTypeEnum value and oaType.getName() function, returns the type name of the object. For example, a shape created using oaRect::create((attributes)) function will have enumeration type (oacTypeEnum) of oacRectType and enumeration value of Rect. Similarly shapes created using oaPolygon, oaPath and oaPathSeg classes will have enum values of "Polygon, Path, and PathSeg" respectively.

Once the type of shape has been identified using enum values, geometric coordinates of these shapes can obtained by querying appropriate attributes associated with the shape. Since layouts are mostly designed using rectangles and closed orthogonal polygons, these two shapes are the most important. Digital integrated circuits contain only these two shapes. The coordinates of a rectangle is usually its bounding box, therefore oaShape.getBBox(oaBox) function can be used to get the $\left(X_{l l}, Y_{l l}\right)$ and $\left(X_{u r}, Y_{u r}\right)$ of the rectangle. Here 11 represents lower left corner and ur represents upper right corner. Similarly, the number of corner points in the polygon can be obtained by using oaShape.getNumPoints() function and oaShape.getPoints(oaPointArray) returns the collection of corner points, i.e., (x, y) coordinates, which describes the polygon.

During the course of the work, it was observed that OpenAccess API doesn't have any functions or classes that stores the height or thickness of a layer. This is a critical information for modeling the shapes three dimensionally. Chapter 8 discusses converting $2 \mathrm{D}$ rectangles to $3 \mathrm{D}$, for which polygons need to be fractured in to rectangles which is discussed in Chapter 7 


\subsection{Query Pseudocode}

In this section a pseudocode that is used to implement the query to get geometric information of all the shapes in the design is presented.

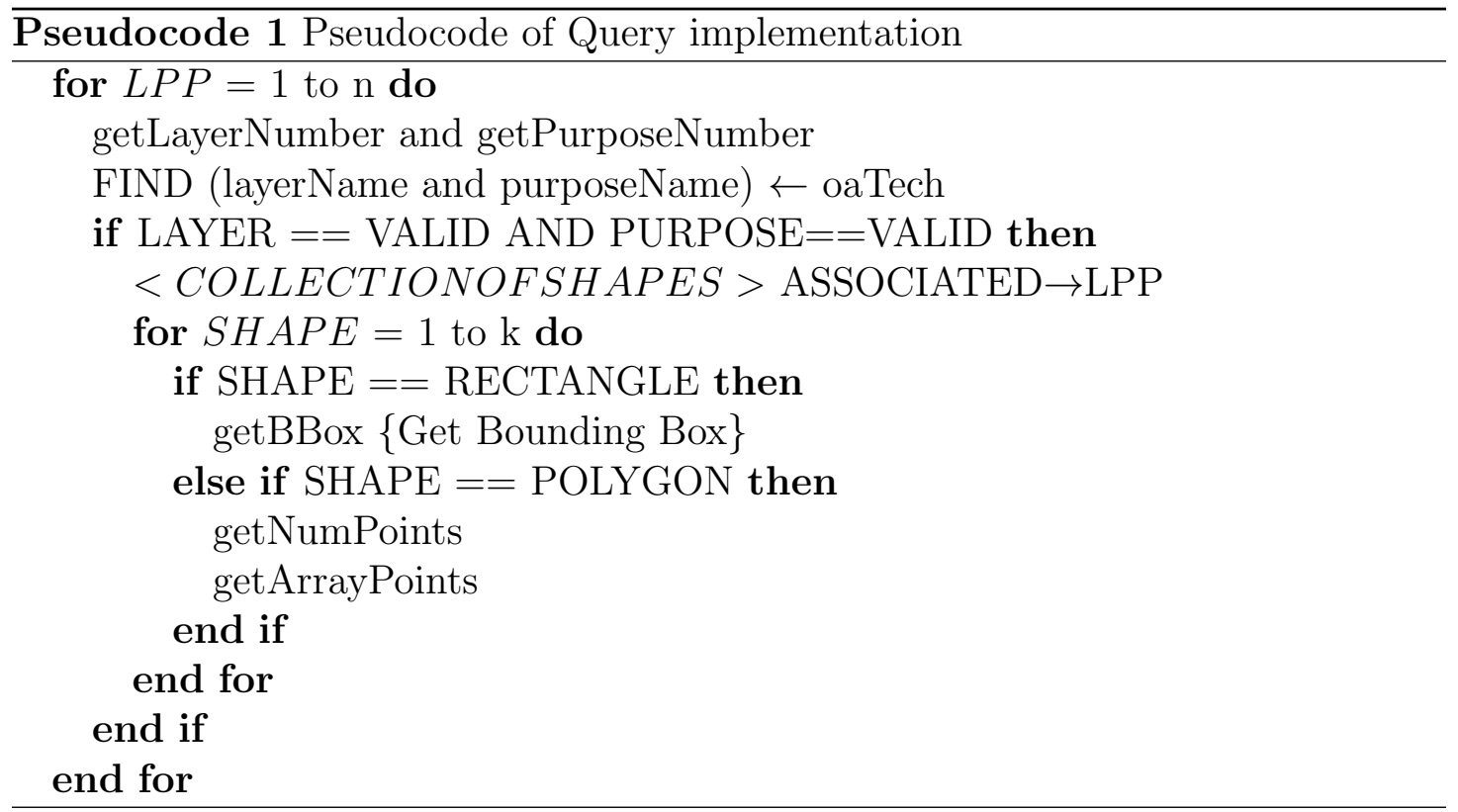




\section{Chapter 7}

\section{Polygons to Rectangles}

From the previous discussions it is clear that OpenAccess supports different shapes and each shape has a different set of attributes. Rectilinear polygons and rectangles are the shapes most commonly used in IC layouts. Chapter 8 discusses an algorithm to model 3D shapes from 2D coordinates using .obj files. Since the .obj file format does not directly support modeling rectilinear polygons, a polygon to rectangle conversion is required. There are other advantages in converting polygons to rectangles. Since rectangles and cubes are primitive shapes in Art of Illusion, rendering rectangles and cubes is faster than rendering polygons. Also, many IC mask-masking applications convert polygons into rectangles before generating the mask. Therefore interconnect data that is being visualized would give the designer a more realistic view of the interconnect as it would appear on silicon.

The work presented in $[19,6,20]$ discusses different algorithms to convert orthogonal polygons to rectangles. All these algorithms are specifically addressed for VLSI CAD applications. Nahar et al.[19] presents a fast algorithm to decompose orthogonal polygons to rectangles. This algorithm uses only horizontal cuts to report non overlapping rectangles and assumes that the input polygon is sorted anti-clockwise. Lu et al.[20] presents a steiner-tree based approach to partition 
polygons into rectangles. This algorithm uses is based on minimal partitioning tree(MPT). A heuristic algorithm is also presented to construct the MPT. Another polygon presented by Gourley et al. [6] uses an iterative algorithm to partition orthogonal polygons to rectangles. Since the main objective of the research is to visualize interconnect, the choice of the algorithm used in this research was based on the simplicity of the algorithm. Further performance tuning can be done once the primary objective is implemented. The algorithm presented by Gourley et al. is simple and fast enough. Hence this algorithm was chosen for this work. The algorithm was slightly modified to get better results.

\subsection{PTR Algorithm}

The PTR algorithm is an iterative algorithm. Polygons are usually stored as set of corner points in an array. Upon each iteration, this array is modified and with each pass a rectangle is generated. Following steps describes the algorithm:

- Let $A_{i}$ be the array containing set of corner points $P_{n_{i}}$.

- Point $P$ represents a corner point of the Polygon, (X, Y) and $i$ represents that the array changes for each iteration.

- $P_{k_{i}}$ is the leftmost of the lowest points in the array $A_{i}$.

- $P_{l_{i}}$ is the next leftmost of the lowest points in the array $A_{i}$

- $P_{m_{i}}$ is the leftmost of the lowest points in the array $A_{i}$ with $Y_{m_{i}} \geq Y_{k_{i}}$ and $X_{k_{i}} \leq X_{m_{i}}<X_{l_{i}}$

- Once the above points are identified,

Rectangle $R_{i}$ is represented as $\left(X_{L L_{i}}, Y_{L L_{i}}, W_{i}, H_{i}\right) . W$ and $H$ are width and 
height of the rectangle.

$$
\begin{aligned}
& W_{i}=X_{l_{i}}-X_{k_{i}} \\
& H_{i}=Y_{m_{i}}-Y_{k_{i}} \\
& \left(X_{L L_{i}}=X_{k_{i}}\right) \\
& \left(Y_{L L_{i}}=Y_{k_{i}}\right)
\end{aligned}
$$

- Points $P_{k_{i}}$ and $P_{l_{i}}$ are removed from the array $A_{i}$. If the points $\left(X_{k_{i}}, Y_{m_{i}}\right)$ and $\left(X_{l_{i}}, Y_{m_{i}}\right)$ are present in the array then they are removed from the array, if not these points are added to the array. This new array $A_{i+1}$ is used for the next iteration.

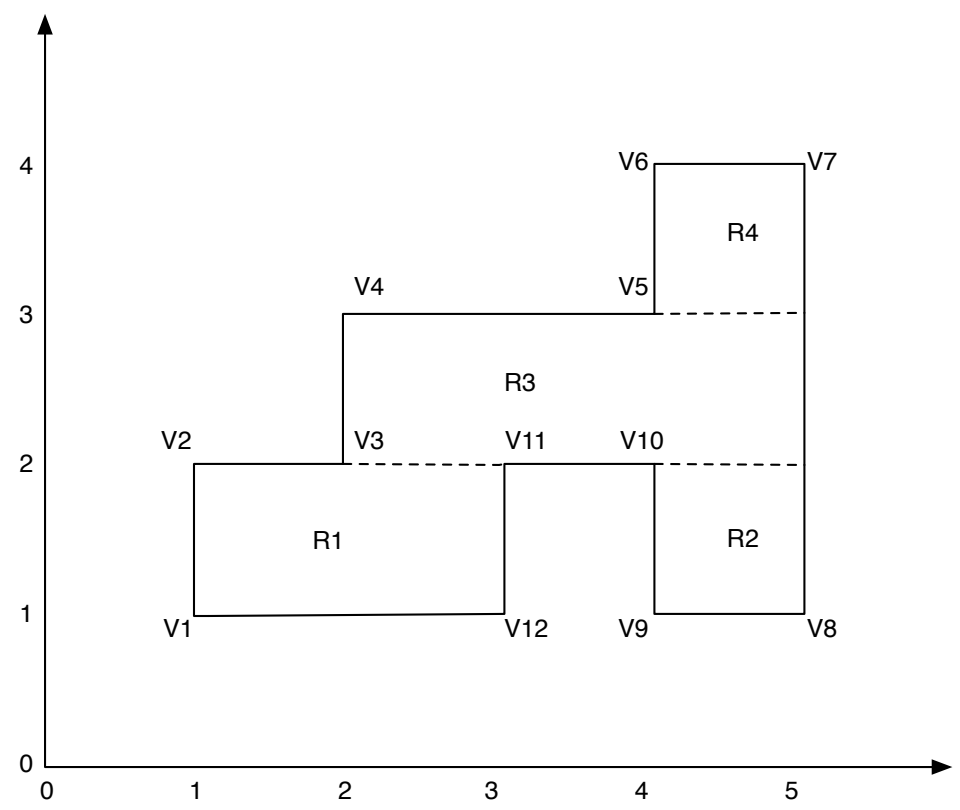

Figure 7.1: Example of PTR algorithm[6]

The above steps are repeated until the array $A_{i}=[$ empty]. The figure 7.1 shows an example of this algorithm. In the figure $V 1, V 2 \ldots V 12$ represents the corner 
points or vertex coordinates of the polygon.According to the algorithm polygon is fractured into four rectangles (R1toR4).

\subsection{Modified PTR, m-PTR Algorithm}

Consider figure 7.1. According to the PTR algorithm, during the 1st iteration $P_{k_{i}}$ is the Point $V 1, P_{l_{i}}$ is the Point $V 12$ and $P_{m_{i}}$ is the Point V2

During the second iteration $P_{k_{i}}$ is the Point $V 9, P_{l_{i}}$ is the Point $V 8$ and $P_{m_{i}}$ is the Point $V 10$

It was observed that during second iteration, if $P_{k_{i}}$ was chosen as $V 3$ and $P_{l_{i}}$ as $V 10$ and $P_{m_{i}}$ as $V 4$, then the number of rectangles representing polygon could be reduced. In this case the total number of rectangles would be three as opposed to four. This is shown in the figure 7.2. From the above discussion, the

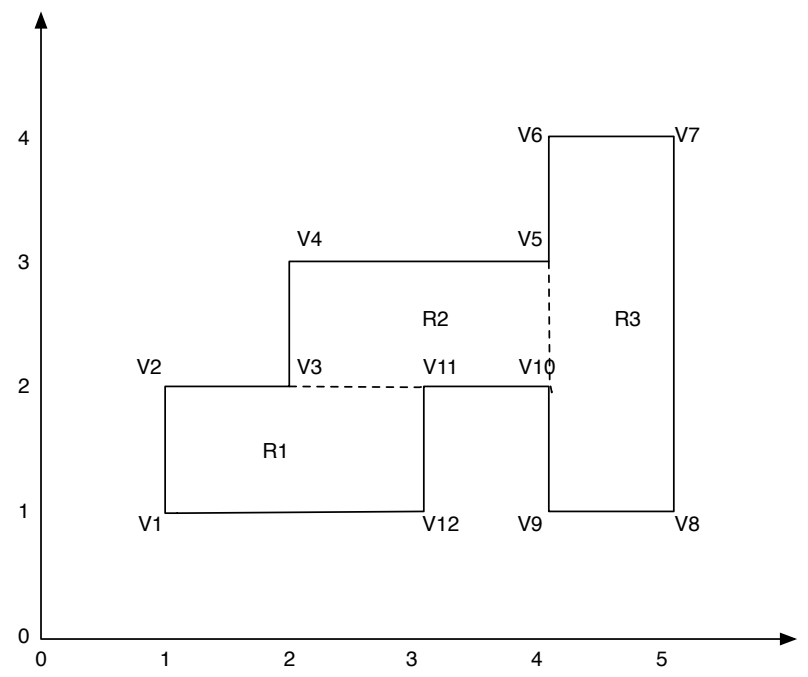

Figure 7.2: Partitioning Polygon to Rectangles using the m-PTR algorithm

definition of $P_{k_{i}}$ and $P_{l_{i}}$ can be modified as leftmost and next leftmost points in the array, respectively. However the definition of $P_{m_{i}}$ remains the same. Making 
this alteration has resulted in three rectangles instead of four. This also reduced the number of iterations required.

Let us consider few more examples to check if this analogy is true. Figure 7.3 shows different polygonal shapes that might occur in the layout most frequently. Table 7.1 compares the number of rectangles generated for the polygons shown in the figure using the PTR algorithm and the m-PTR algorithm. Clearly, in the first two cases the m-PTR algorithm produces better results than the PTR algorithm. However in the last case, both the algorithms produce the same number of rectangles. From the four examples, the best case improvement is $33 \%$ and the worst case is $0 \%$ or no improvement at all. The worst case polygonal shape is usually found in power and ground rails and occurs not as frequently as the other polygonal shapes. Assuming that the m-PTR algorithm provides better results three out of four times, this algorithm is implemented here to decompose orthogonal polygons in to rectangles. Once the conversion is done, representing rectangles three dimensionally becomes a trivial problem which is discussed in Chapter 8. It is important to note that the decomposed rectangles are never written back to the database. Hence this does not affect any other tool operating on the same data.

\begin{tabular}{|c|c|c|c|}
\hline Reference Figure & PTR algorithm & m-PTR algorithm & Improvement \\
\hline Figure 7.3.a & 3 & 2 & $33 \%$ \\
Figure 7.3.b & 4 & 3 & $25 \%$ \\
Figure 7.3.c & 3 & 3 & $0 \%$ \\
\hline
\end{tabular}

Table 7.1: Comparison of PTR and m-PTR algorithm for different polygons 


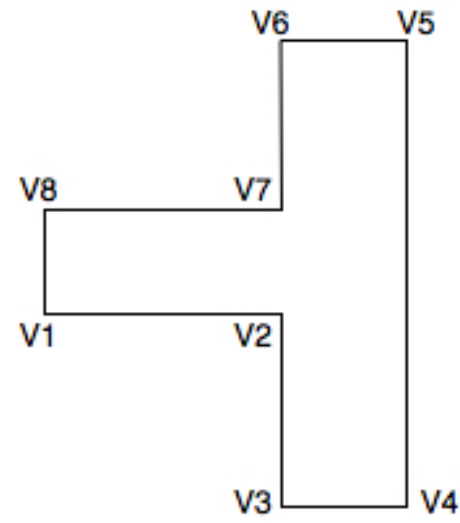

(a)

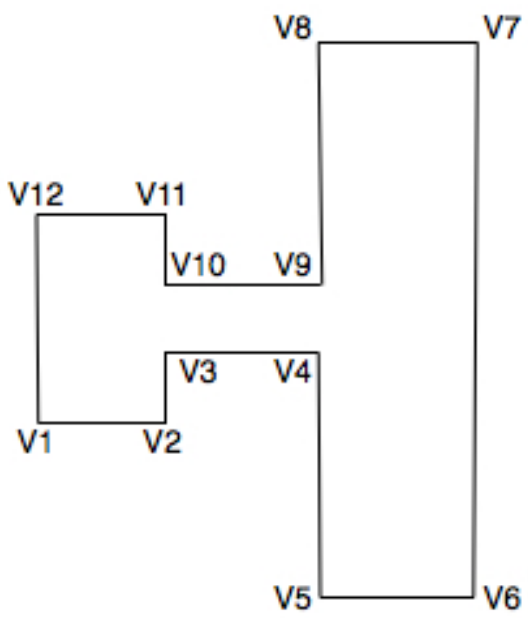

(b)

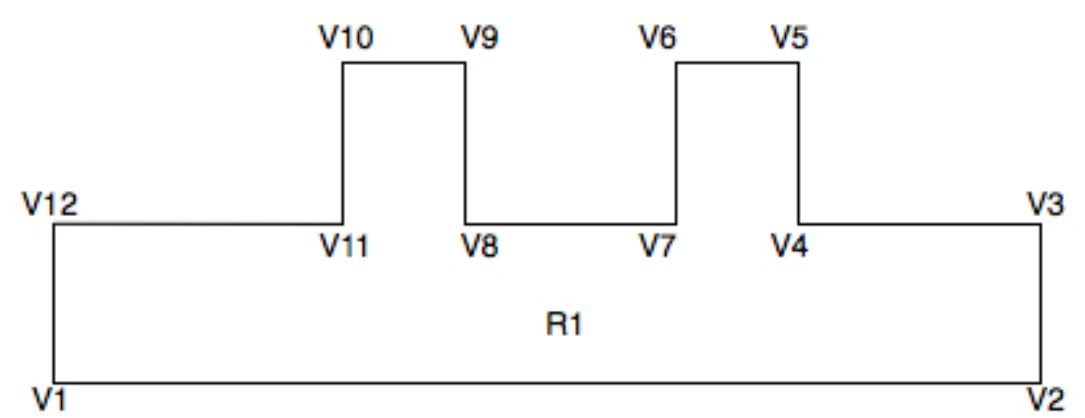

(c)

Figure 7.3: Polygon Examples 


\section{Chapter 8}

\section{$2 \mathrm{D}$ to $3 \mathrm{D}$ conversion}

It is clear that one can query the $\mathrm{x}$ and $\mathrm{y}$ coordinates of rectangles and polygons from the OpenAccess design database. Modeling these shapes three dimensionally requires the third, $\mathrm{z}$, coordinate. In case of interconnect, the third dimension is its thickness. The thickness of the wire depends on the process technology. To get the thickness of the wire, the technology provider needs to include this information in the technology file or a database and the database API must provide access to this information.

The OpenAccess based design used to test the work, provided technology information in both ASCII file format (.tf) and as OA tech database. It was observed that the technology file had no information regarding layers thickness. More importantly the layer thickness is not modeled in OpenAccess. Unless the layer thickness is assumed, viewing IC interconnect three-dimensionally is impossible. Hence in this work, the thickness of the metal layers and vias were assumed.

Recall that all the orthogonal polygons in the layout are fractured into rectangles and in Chapter 7 an algorithm to implement that was also presented. Hence the only shape that needs to be modeled in $3 \mathrm{D}$ cartesian are rectangles. In the 
following sections, an algorithm to convert a 2D shape to a 3D shape, file formats to represent 3D objects, their texture information and the pseudocode of the algorithm are offered.

\subsection{D and $3 \mathrm{D}$ cartesian}

Figure 8.1 shows a $2 \mathrm{D}$ rectangle and a $3 \mathrm{D}$ cube. In a 2D domain, a rectangle can be modeled with just two corner points, lower left corner $\left(X_{l l}, Y_{l l}\right)$ and upper right corner $\left(X_{u r}, Y_{u r}\right)$ points. This is shown in figure 8.1(a).

To model 2D rectangles as 3D objects in Art of Illusion, the primitive cube object type with appropriate length, width and height can be used. Figure 8.1(b) shows a 3D cube modeled in AOI. A simple 3D cube or a 3D rectangle will have 8 vertex coordinates and 6 faces. The .OBJ file format presented in the Section 8.2, uses this vertex and face information to represent $3 \mathrm{D}$ objects. A 3D cube can also be represented with just two corner points. However in this case the z-axis coordinates $\left(Z_{l l}, Z_{u r}\right)$ are also needed, i.e, $\left(X_{l l}, Y_{l l}, Z_{l l}\right)$ and $\left(X_{u r}, Y_{u r}, Z_{u r}\right)$.

Note that, from the figures 8.1(a) and 8.1(b), the top view in 2D cartesian coordinate system is the front view in the $3 \mathrm{D}$ plane. Therefore the $y$-axis in the $2 \mathrm{D}$ cartesian represents the $z$-axis in the 3D cartesian. Now the $y$-axis in the 3D plane represents the height or the thickness of the object (in this case, interconnect). Therefore the y coordinates of a $2 \mathrm{D}$ rectangle becomes the $\mathrm{z}$-coordinates of a $3 \mathrm{D}$

rectangle, i.e., $\left(Z_{l l}, Z_{u r}\right)=\left(Y_{l l}, Y_{u r}\right)$ and the new $\left(Y_{l l}, Y_{u r}\right)$ represents the height of the $3 \mathrm{D}$ rectangle.

The interconnect are multilayered (poly, metal1 etc) with contacts and vias(via1, via2 etc). Each layer's height or thickness may vary, as shown in figure 8.2. From 


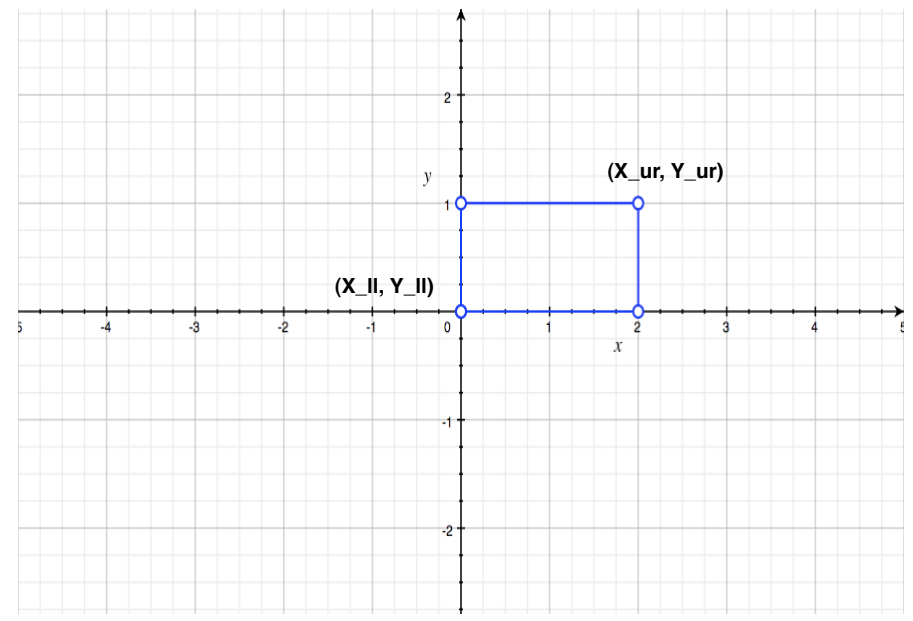

(a)

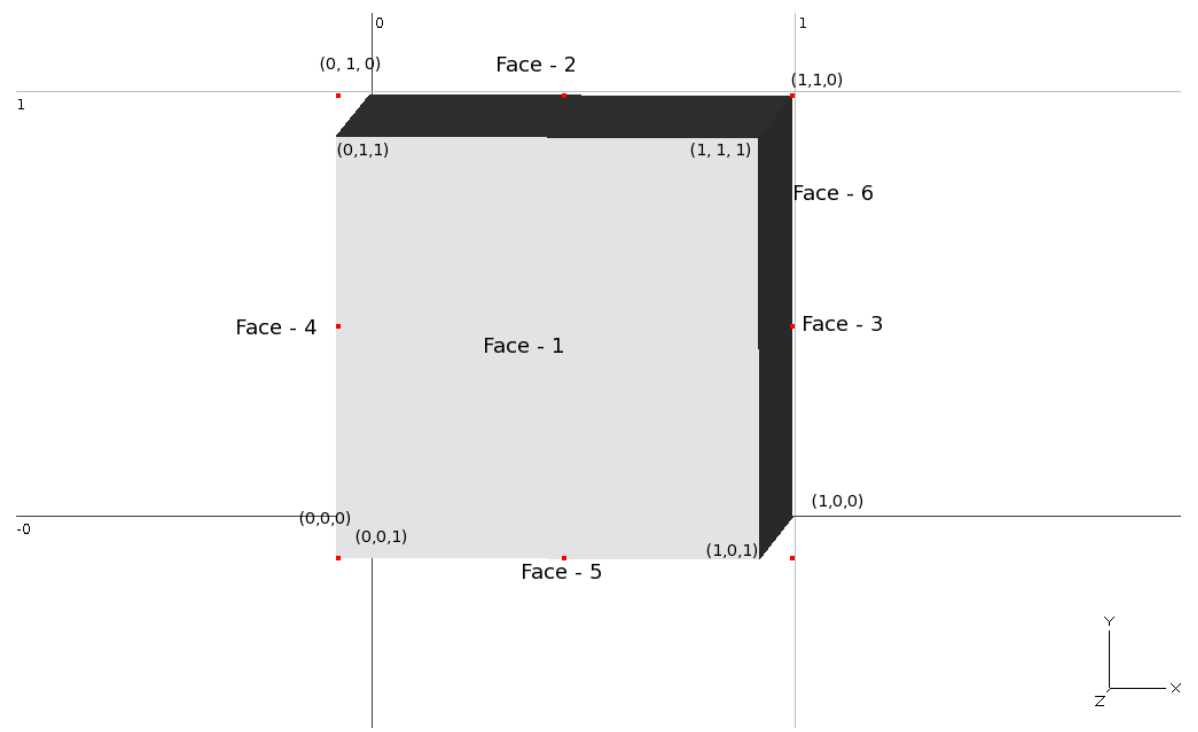

(b)

Figure 8.1: Figure showing 2D and 3D cartesian 


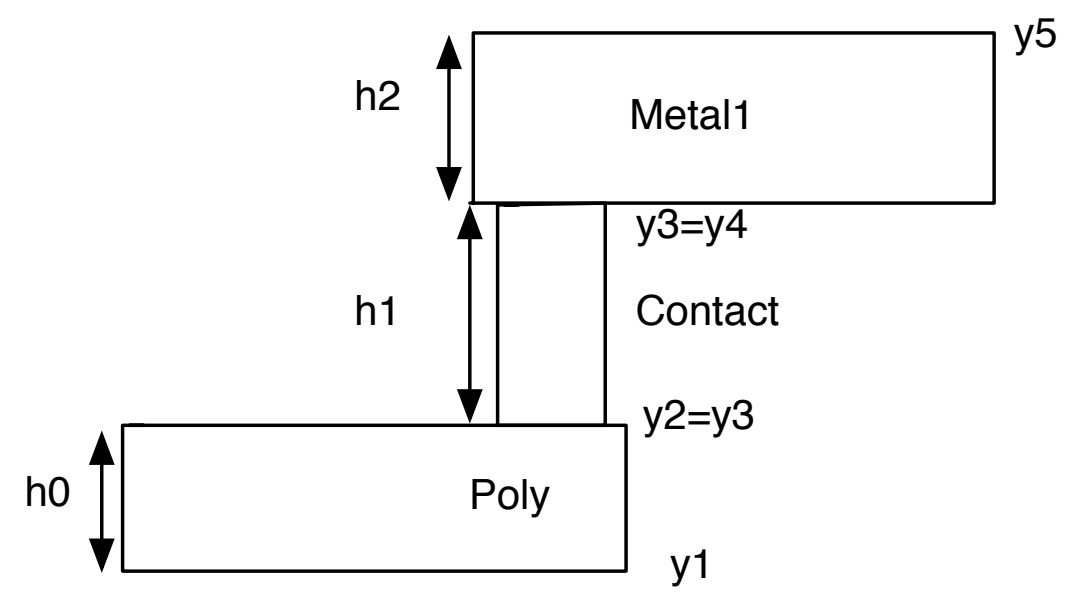

Figure 8.2: Computing layer thickness

the figure, h0, h1 and h2 are the thickness of poly, contact and metal1 layers respectively and $y 1 \ldots y 5$ are the $y$-coordinates to represent that thickness. If the height of each layer is assumed, from the figure 8.2, the $y$-coordinates can be calculated as follows:

$\mathrm{y} 1=0, Y_{l l}$ of Poly layer (lowest layer)

$\mathrm{y} 2=\mathrm{h} 0=\mathrm{y} 3, Y_{u r}$ of Poly layer and $Y_{l l}$ of Contact.

If Poly is the lowest interconnect layer, then for $n$ layers, this can be generalized, shown in Algorithm 2:

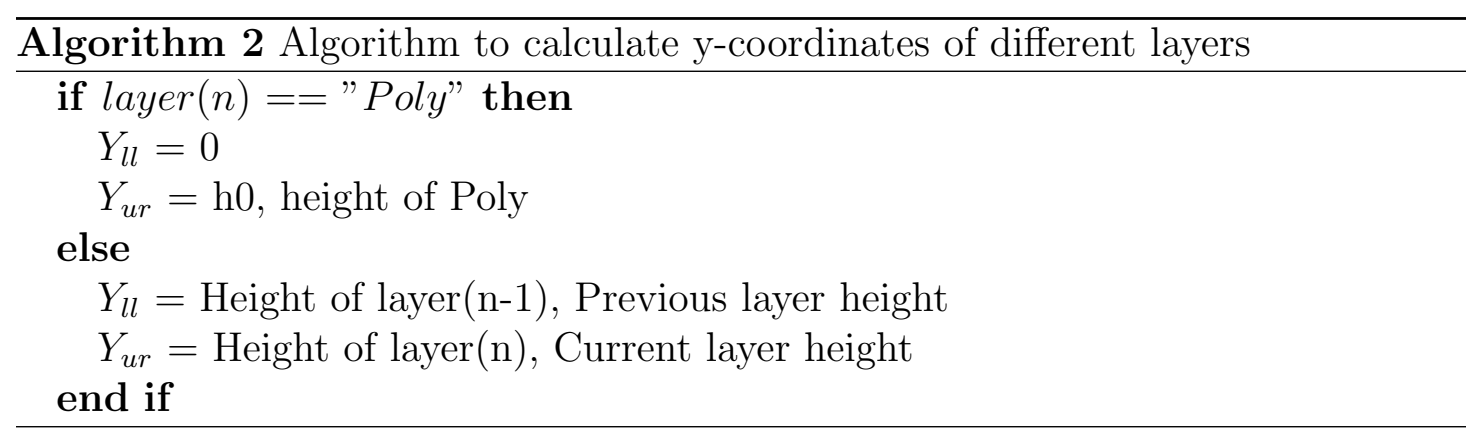

Therefore once the appropriate $\mathrm{y}$-coordinates are calculated, the 2D rectangles 
can be represented as 3D objects by generating 8 vertices and 6 faces and writing it to .OBJ file. The .OBJ file format is discussed next and an algorithm to represent 3D object is shown in Algorithm 3.

\section{2 .OBJ and .MTL file formats}

\subsection{1 .OBJ File Format}

The.$O B J$ file format is used to store the $3 \mathrm{D}$ description of objects. This file format was developed for the Advance Visualizer package by Wavefront Technologies [21]. The file contains information about each objects's vertex coordinates, texture information of each vertex, vertex normals and faces that make polygons. Following is an example of .OBJ file format that represents a simple 3D rectangular cube. The two corner points of this cube are $(0,0,0)$ and $(0.5,0.25,0.5)$

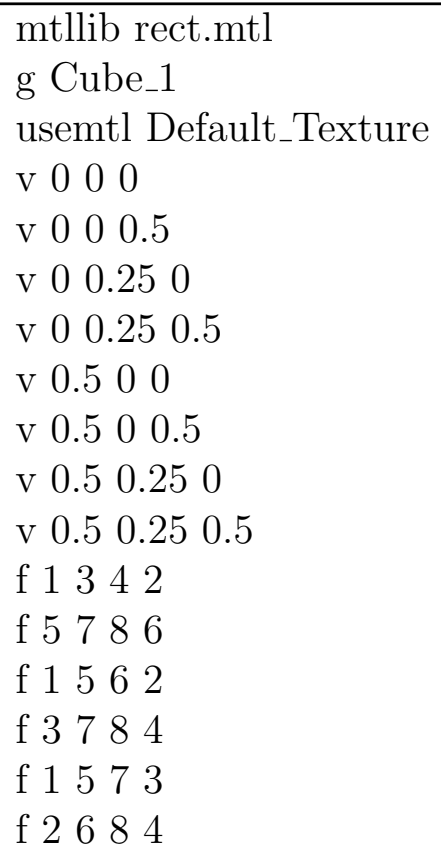

f 1562

f 3784

f 1573

f 2684

In the above example 
- mtllib statement specifies the material library file. This library file contains material and texture description that are used to make the model look more realistic. This is an optional statement.

- $\mathrm{g}$ is the group statement. This is used to organize the collection of elements.

- usemtl statement specifies the material name to be used for the following objects. If no material name is specified white is used by default. Again this statement is also optional.

- Statements starting with $\mathrm{v}$ are vertex data. It specifies a geometric vertex and its $\mathrm{x}, \mathrm{y}$ and $\mathrm{z}$ coordinates.

- Statements starting with $\mathrm{f}$ are face data. It represents a polygonal geometry. A face of a polygon is constructed from the vertex reference numbers. In the above example, f 1342 statement uses vertex numbers 1,3,4 and 2 to construct a rectangular face.

The figure 8.3 shows the above example modeled in Art of Illusion. More detailed information about .OBJ file format is given in Appendix A.

\subsection{2 .MTL File Format}

The .MTL file format is usually used as an auxiliary file with .OBJ files [22]. This file holds material descriptions, such as ambient color, diffusion color, specular color, transparency, shininess, illumination etc., of the material. If no Material Library file is specified in the .OBJ file, all objects represented in the file will have "white" color. Following is an example of .MTL file, describing the material color and illumination model. 


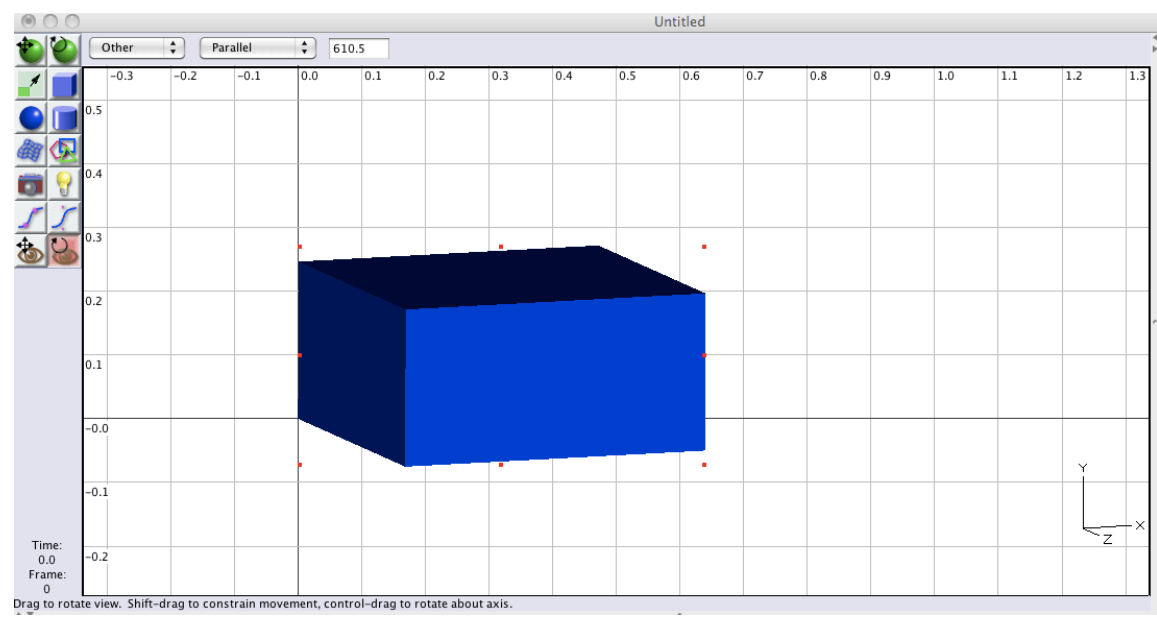

Figure 8.3: A 3D rectangle, modeled in Art of Illusion

newmtl Red

Kd 100.0

Ks 0000

Ka 000

illum 1

- Each material definition begins with a newmtl syntax.

- Ka defines the ambient color of the material.

- Kd defines the diffuse color of the material.

- Ks defines the specular color of the material.

- illum defines the illumination model used by the material.

For the work presented in the document, the .MTL file is used so that each layer can be modeled differently and distinguishing between the layers is easier. Following is the .MTL file used for this research. More detailed information on .MTL file format is documented in Appendix B, which can be used to make more realistic objects. This is very useful when visualizing current density and voltage distributions. For 
newmtl poly

Kd 100

Ks 0000

Ka 0000

illum 1

newmtl contact

Kd 111

Ks 0000

Ka 000

illum 1

newmtl metal1

Kd 0001

Ks 0000

Ka 000

illum 1

example, a net or via with high current density or IR drop, can be represented with bright red color whereas other nets may be represented with magenta or blue colors.

\subsection{D to $3 \mathrm{D}$ pseudocode}

In this section, the pseudocode to represent $2 \mathrm{D}$ rectangles as $3 \mathrm{D}$ objects is shown. The inputs to his algorithm are $(\mathrm{x}, \mathrm{y})$ coordinates of the rectangle, layer height and layer name. Recall that layer height and layer name are required because each layer can have different thickness. It also requires a file pointer for writing 3D object description. For simplicity file information is not shown in the pseudocode. The layer height is obtained from the Algorithm 2. The material information for the object can be included, it is optional. If no material information is specified, white is used. 


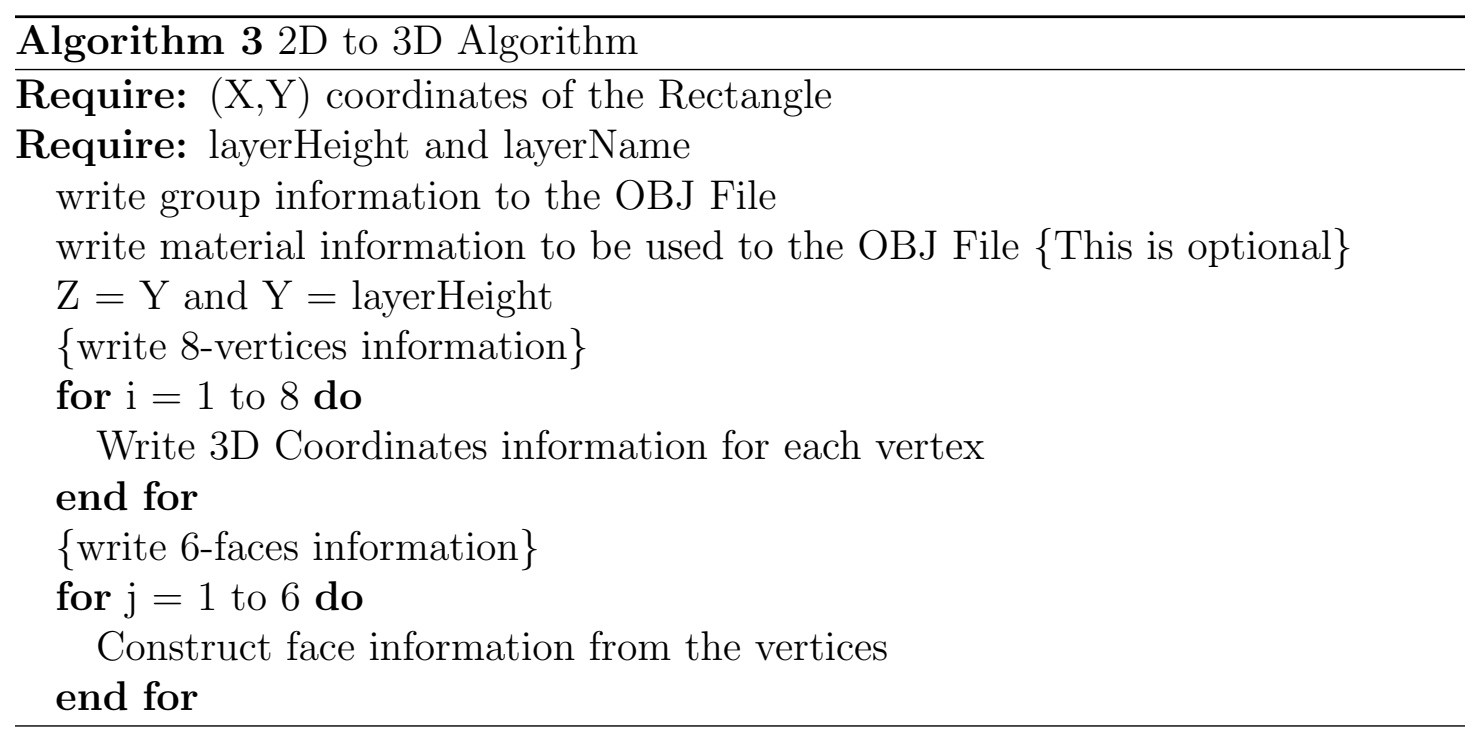




\section{Chapter 9}

\section{Eclipse Platform for Tool Integration and Deployment}

The need to integrate various tools to a common platform was discussed in section 2.2. In this section one such platform, Eclipse, is presented.

\subsection{Eclipse}

Eclipse is an open source, Java based software development platform comprising an IDE (Integrated Development Environment). Eclipse is mainly written in Java and supports various programming and scripting languages like $\mathrm{C} / \mathrm{C}++$, Python, Shell, Perl and other languages through plugins. Eclipse is mainly built on plugin architecture, i.e, aside from a small runtime kernel which is responsible for launching eclipse and managing the resources, everything else in Eclipse is a plugin. Plugins can extend the functionality of other plugins or provide new feature to eclipse. Eclipse also includes debuggers to help developers debug the code. Eclipse is not just an IDE, it also provides framework for integrating different tools into a single integrated application. Yang and Jiang [23] compares Eclipse with different tools like IntelIJ IDEA, Netbeans and XML-Based integrator. From this work Eclipse clearly has the upper hand in terms of number of plugins available, programming languages supported and support for different levels of integration. 
This research mainly uses Eclipse as a development environment. However a study on Eclipse for tool integration and deployment was done and is presented in the subsequent sections.

\subsection{Eclipse for Tools Integration}

Integrating tools with the Eclipse platform reduces the cost of the integration, since Eclipse provides various services, APIs and frameworks that facilitates scalable integration. This includes user interface frameworks such as JFace and Swing, component version management etc.

The work presented by Amsden [24] shows five different ways to integrate tools with Eclipse depending on the level of integration required. The API, Data and UI integration are three among the five.

- Data Integration allows integration at data level, that is, the data manipulated by one tool is available to other tools. This type of integration requires an access method, an interchange protocol and data transformation facilities.

- API Integration allows other tools to access the application's data via tool specific APIs. This may be preferred when tools operate on each other data and update them. Eclipse supports only tool specific Java APIs. Tools written in other languages must provide a java interface through JNI. Tools integrated via the API run on same Java Virtual Machine and data can be exchanged securely.

- UI Integration allows different tools to share the same user interface as 
if they were designed as a single application. This is accomplished by integrating the tool with the Eclipse Workbench using Eclipse UI framework. The Eclipse Workbench provides extension points to add new views, editors, perspectives, splash screens etc. Also, UI Integration technique allows tools to register interest in events generated by other tools.

A possible outcome of UI and API integration of Schematic Editors, simulators, layout editors etc is shown in figure 9.1.

The simplest way that a tool can integrate with Eclipse is to integrate with the Eclipse Workbench UI. The Workbench UI is comprised of views, editors, perspectives, actions and preferences. Each individual component of the UI can be customized by extending the org.eclipse.ui plugin. For example a new view can be created by extending the org.eclipse.ui.views plugin. Note that the org.eclipse.ui.views plugin is itself an extension of the org.eclipse.ui plugin. The layout of new views and editors can be customized by creating a new perspective. For example figure 9.1(a) shows a new perspective for a spice simulator, comprising of views showing a design library, a waveform viewer, errors log and a schematic editor.

Integrating the OpenAccess API and other tools used in the Analog/Mixed signal design flow with Eclipse can provide seamless exchange of data across different tools. Most of the VLSI design tools are written in $\mathrm{C} / \mathrm{C}++$, hence integrating them with Eclipse can be very challenging. Nevertheless, a unified framework with all the required tools is what most commercial tools lack. 


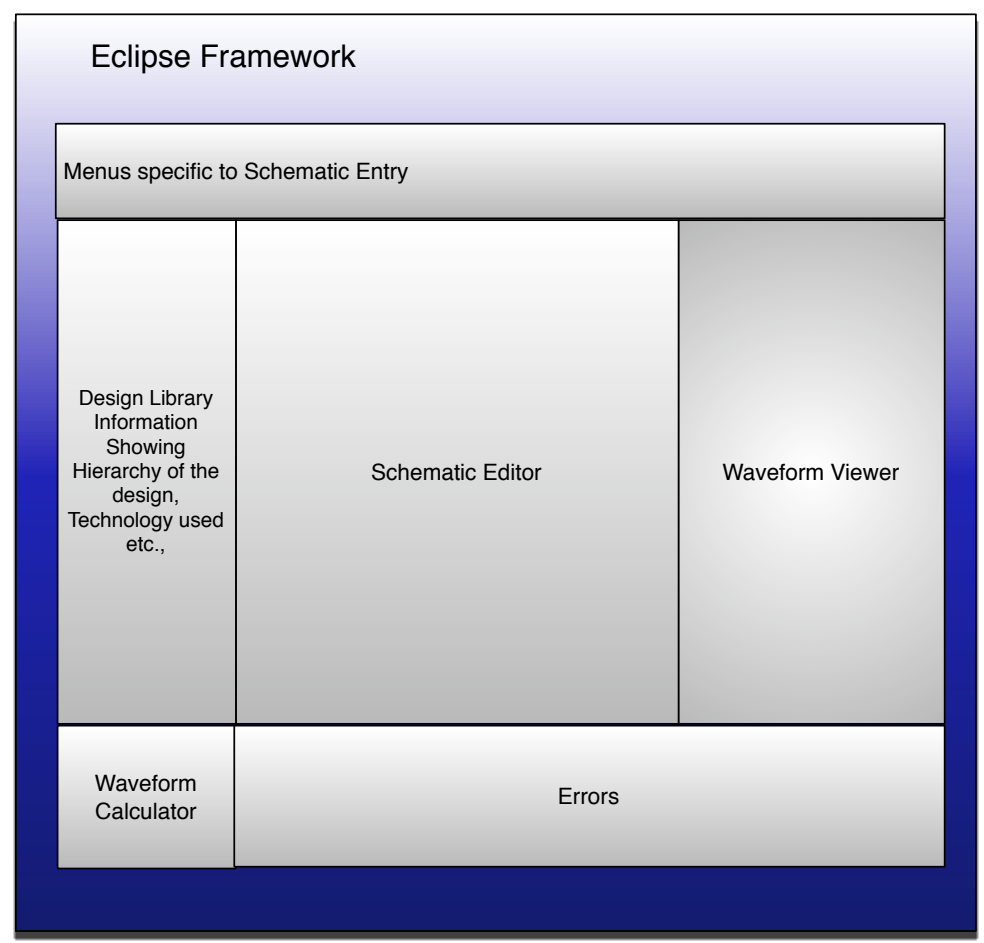

(a)

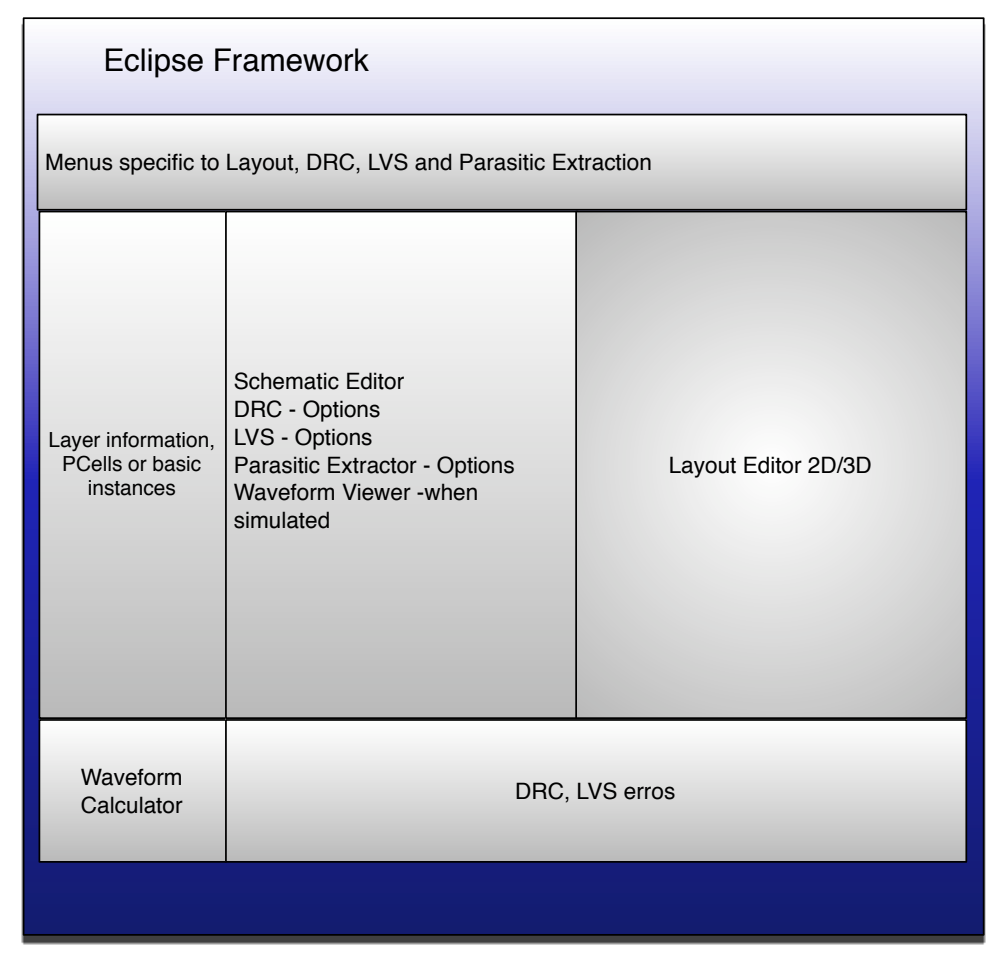

(b)

Figure 9.1: Possible outcome of Integration in Eclipse Framework 


\subsection{Eclipse for Tool Deployment}

In the previous section, Eclipse support for different levels of integration was discussed. Packaging and deploying these tools such that they work well as a single stand alone application in the host environment is essential. Since everything in Eclipse is a plugin, deploying the integrated tools is relatively simple. However care must be taken to ensure that all the dependencies are met. Eclipse simplifies this process by providing a Rich Client Platform (RCP), i.e., it can package a minimal set of plugins required to build a rich client application [25]. It also supports JFace and the Software Widget Toolkit (SWT) UIs to build rich client applications by extending org.eclipse.jface and org.eclipse.swt plugins respectively. The SWT can be used to provide native look and feel to the application whereas JFace extends SWT and provides classes for common UI programming. In Eclipse, JFace depends on SWT but not vice versa.

There are different ways that Eclipse can deploy a rich client application. Eclipse supports deploying applications as archives (.zip, .jar files), native interfaces (InstallShield, RPM, .dmg etc) and Java Web Start (JNLP). The simplest way to deploy is by archiving the necessary plugins like .zip, .jar, .tar etc. This is also the most standard way of delivering the application to the end user. Eclipse can also package the application as native installers like InstallShield(windows), RPM (linux platforms), .dmg (Mac OS) etc. An advantage of this is that the native installers allow the application to tightly integrate with the underlying operating system. An application can also be delivered as a java web start (JNLP) file. The file itself doesn't contain any application code or plugins, instead it contains the link of a web server from where the application can be downloaded. The end 
user need not perform any configuration, since it is all done automatically by the configuration file contained within.

Eclipse is very popular in embedded systems development. Texas Instrument's Code Composer Studio and Freescale Semiconductor's Code Warrior tools were developed using Eclipse.

For this research OpenAccess and Art of Illusion source codes were imported to Eclipse and compiled. The Appendices C and D show the procedure to do this. Also a translator that queries the layout data from OA database and converts it to .OBJ file format was implemented using Eclipse. Since OpenAccess API is a $\mathrm{C}++$ interface, a java interface, using JNI, must be created in order to integrate it with Eclipse.

Integrating Art of Illusion with Eclipse is straightforward, since it is written in Java. To integrate AOI with the Eclipse workbench UI, a new set of java classes that extend org.eclipse.ui plugin to create new views, editors, actions, perspective etc, and provide interface with AOI actions, views, perspectives etc, are required. Besides, integrating AOI with Eclipse is only meaningful if it can integrate tightly with the OA API or if the user interface of AOI needs improvement. Therefore AOI integration with Eclipse was not carried out in this research. 


\section{Chapter 10}

\section{Test results and discussion}

Algorithm 4 shows the complete pseudocode to generate the .OBJ file that contains the 3D description of the circuit's interconnect. In this code the polygon to rectangle algorithm is not elaborated and can be treated as a function that returns a set of rectangles representing the polygon. Similarly the 2D to 3D algorithm can also be treated as a function that writes $3 \mathrm{D}$ rectangle information to the .OBJ file. This function can be treated as type void. The pseudocode was implemented as

a $\mathrm{C}++$ program. The flow chart describing the complete algorithm is shown in figures 10.1 and 10.2 .

The OpenAccess and Art of Illusion source codes were compiled in Eclipse 3.4 IDE on Fedora 10 linux operating system. The version of OpenAccess used was 22_04p021. GCC version 4.1 was used to compile the $\mathrm{C}++$ program and to install the OA reference implementation. The Nangate's OpenCell 45nm Standard Cells were used for testing the algorithm to view the IC interconnect three dimensionally. Synopsys has released a viewer called "oaViewer" which reads OA layouts. It is a Tcl application and Tcl wrappers to OpenAccess must be installed to use this viewer. Installing Tcl wrappers is described in Appendix C. Synopsys oaviewer is used to compare and verify the interconnect viewed in Art of Illusion. 


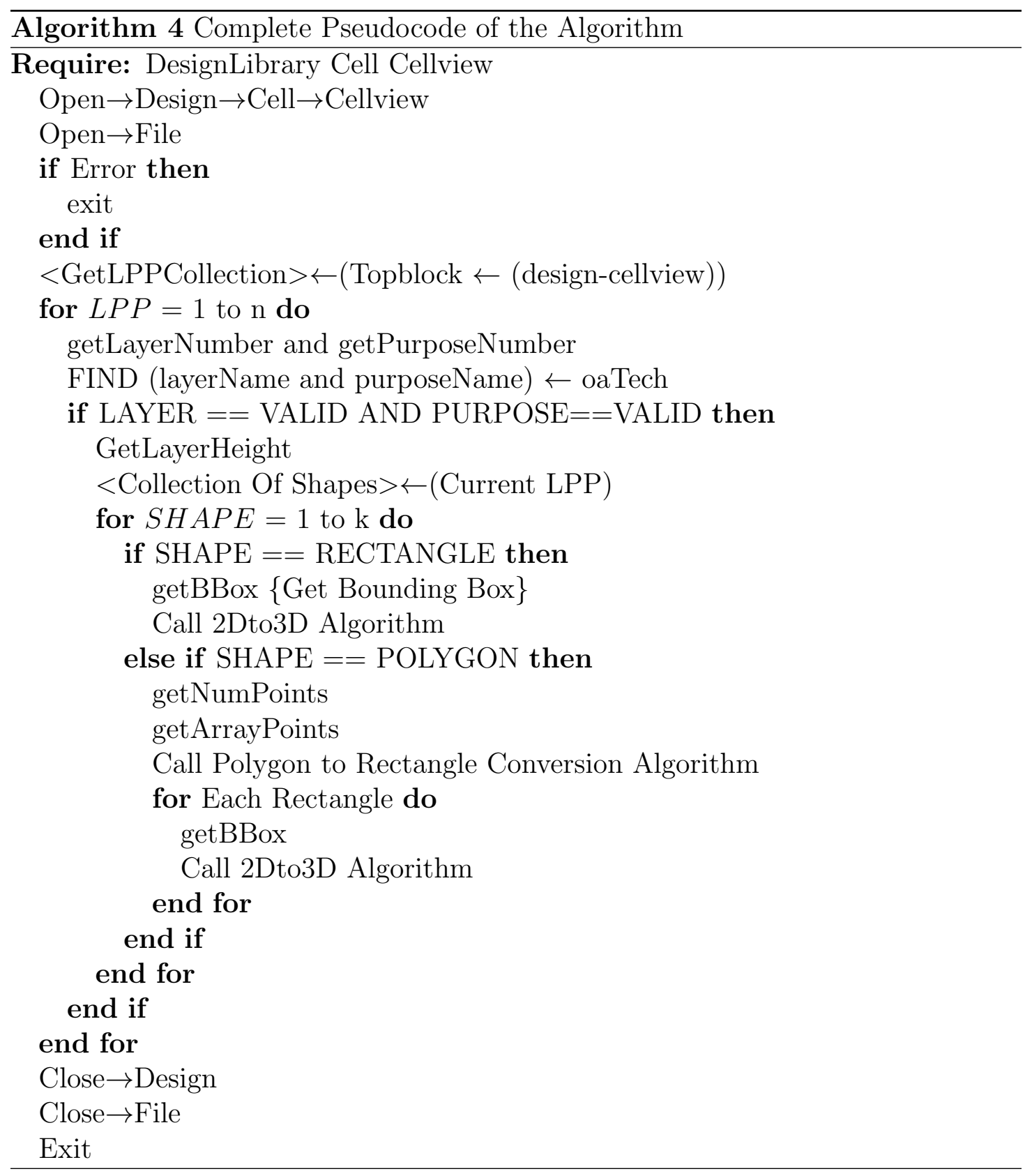




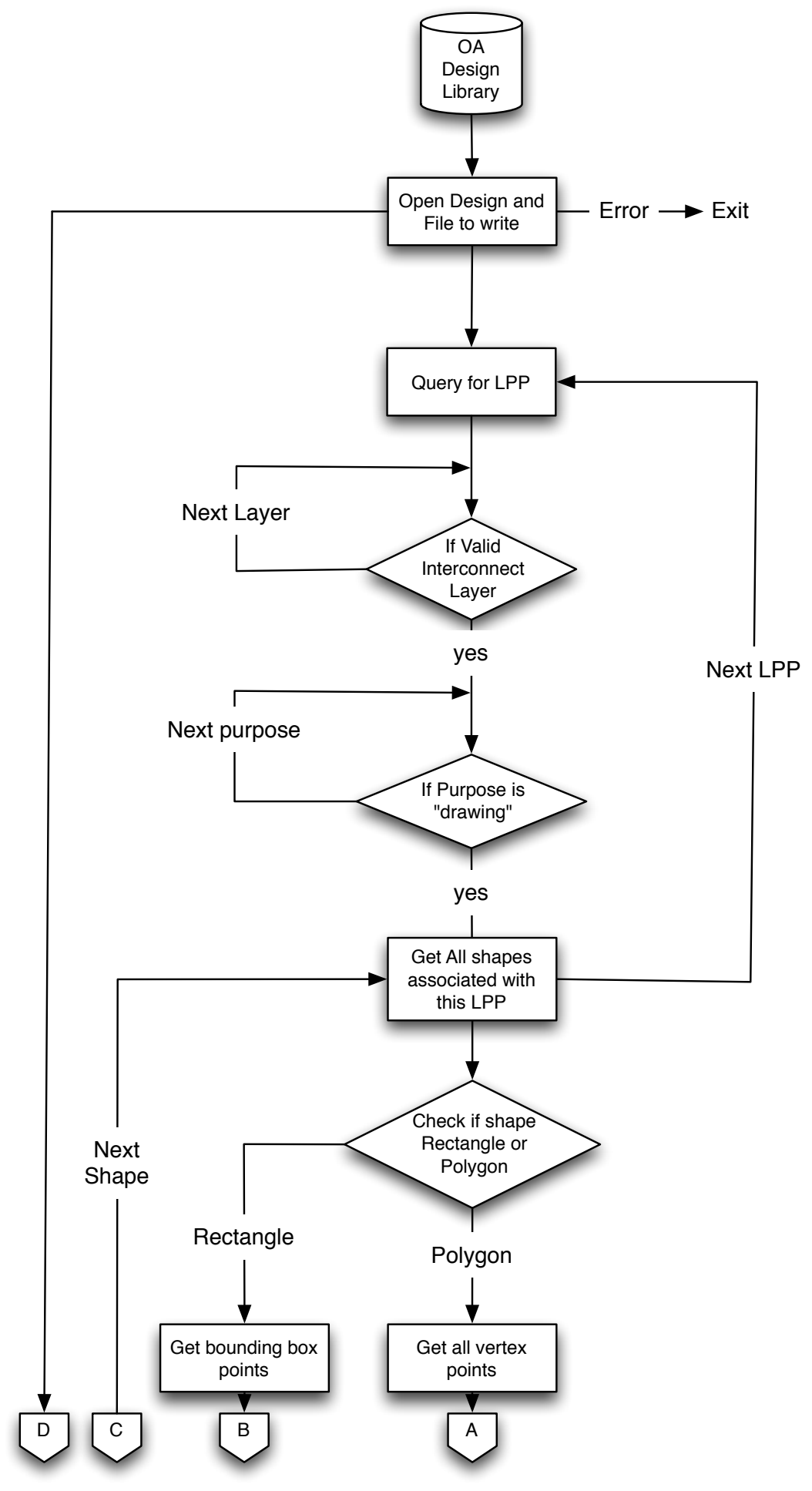

Figure 10.1: Complete Algorithm Flow Chart 


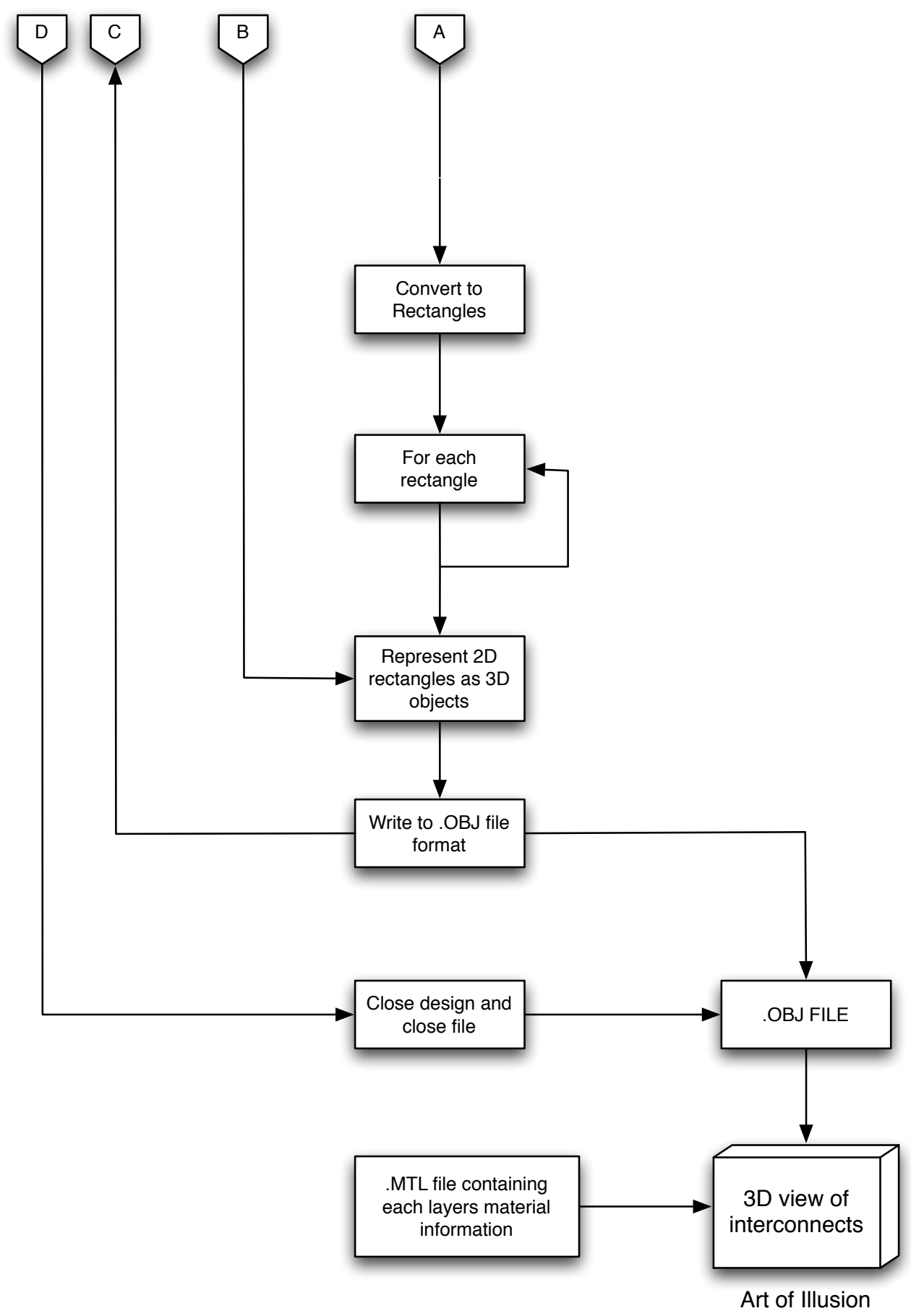

Figure 10.2: Complete Algorithm Flow Chart cont. 
Figure 10.3 shows the layout of a 2 input Nand Gate cell viewed in oaViewer (Fig.10.3(a)) and the top view of the Nand gate interconnect layout in Art of Illusion (Fig.10.3(b)). It can be observed that the interconnect in AOI is mirrored along the z-axis. This is because the top view in Art of Illusion has X-Z coordinate axes. The positive z-axis is pointed inwards. Since $+y$ axis coordinates are mapped to $+\mathrm{z}$ axis, the interconnect layout looks inverted in Art of Illusion. For comparison, the coordinates of + z-axis were mirrored to -z-axis in the program. Figure 10.4 shows the corrected top view of the interconnect modeled in AOI.

Figure 10.5 compares the layout of Full Adder cells in oaViewer and Art of Illusion. The full adder cell was the largest available in the cell library. Figure 10.6 shows the 3D view of the Full Adder interconnect layout.

Figures 10.3 to 10.6 demonstrate the $3 \mathrm{D}$ visualization of IC interconnect using free open source tools. Rendering of these layouts was almost instantaneous and zooming was smooth. When dealing with large designs, speed is of great importance. Since there were no changes made to the source code of Art of Illusion or OpenAccess, their performance remains as claimed. In this case the translation time, i.e., querying the design data from OpenAccess to .OBJ file format is important. The critical functions in the program are File I/O, Design Query and Polygon to Rectangle conversion.

Program execution time can be calculated by calculating the beginning and the end times. The difference between these two times is the execution time. This might not be accurate, if the program takes less than a second to execute. Another approach is to count the number of clock ticks and divide it by an appropriate number. To facilitate this, a C-Library time.h was used. The function clock_t clock(void); returns the number of clock ticks elapsed since the launch of 


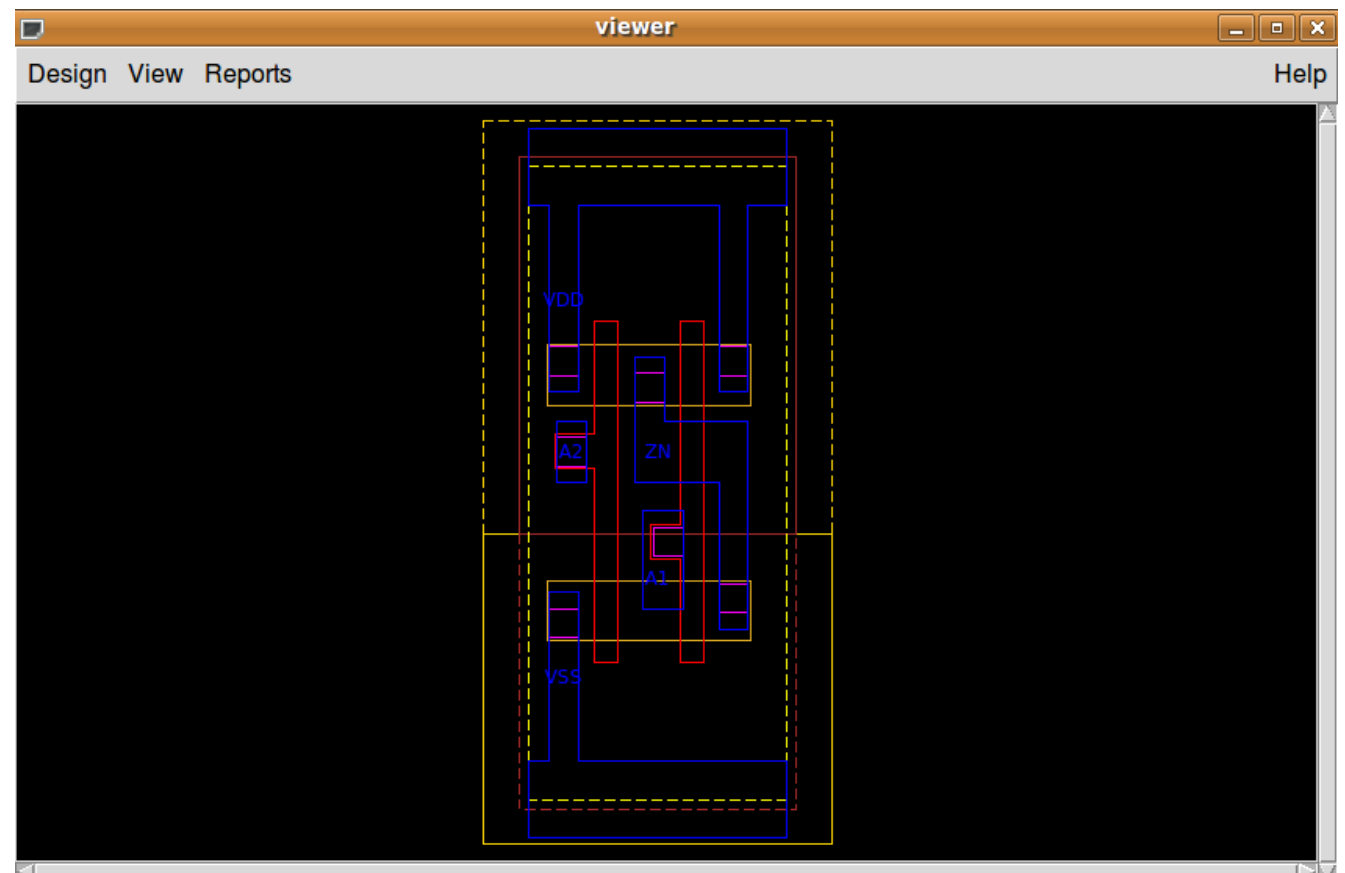

1.74440 .1231 View: NangateOpenCellLibrary_PDKv1_2_vinfo:

(a) Nand Gate Layout in oaViewer

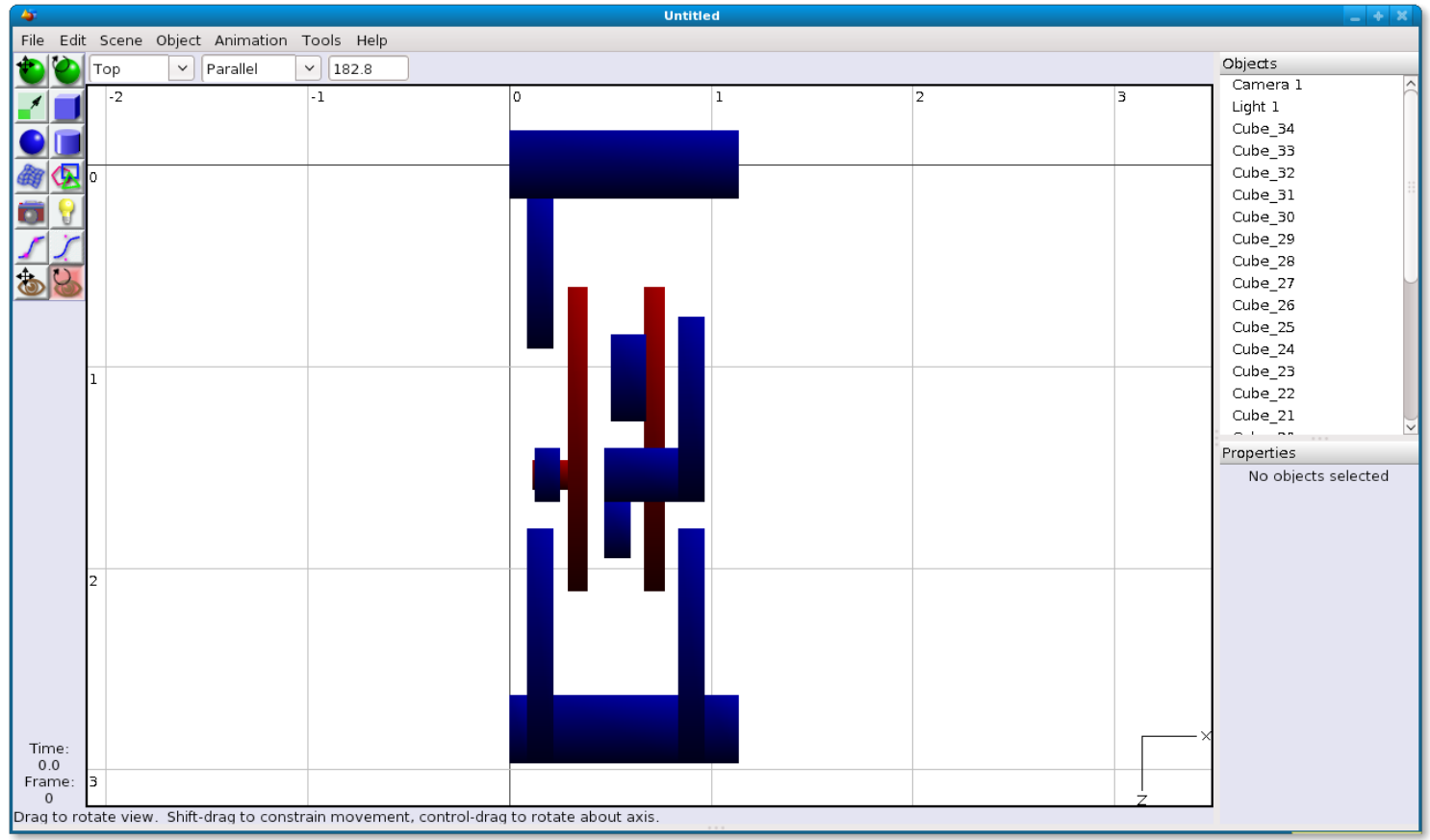

(b) Top View of Nand Gate interconnect in AOI

Figure 10.3: Top view of Nand Gate in oaViewer and Art of Illusion 


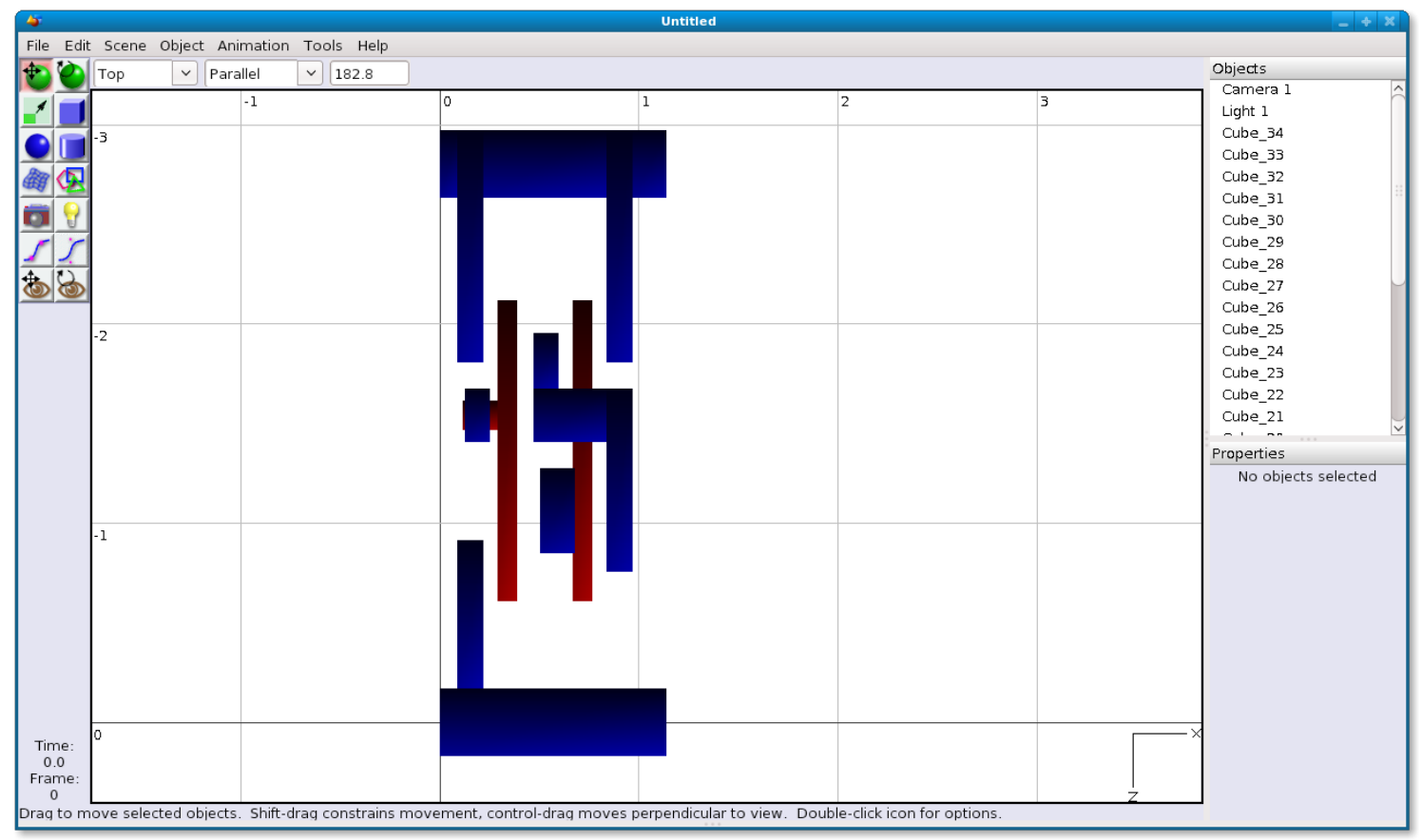

Figure 10.4: Corrected Z-Axis Top View

program, and the macro CLOCKS_PER_SECOND specifies the relationship between clock ticks and seconds. At the beginning of the program the clock tick reading was taken (start) and at the end of the program another clock tick reading was taken(end). Now the difference between these two readings (end-start) divided by CLOCKS_PER_SECOND gives the execution time in seconds. The execution or translation time for three cells NAND2_X1 and FA_X1 is shown in Table 10.1. The results show that the conversion time is in few tens of milli seconds. Clearly, a design with fewer Polygons takes less time for conversion. 


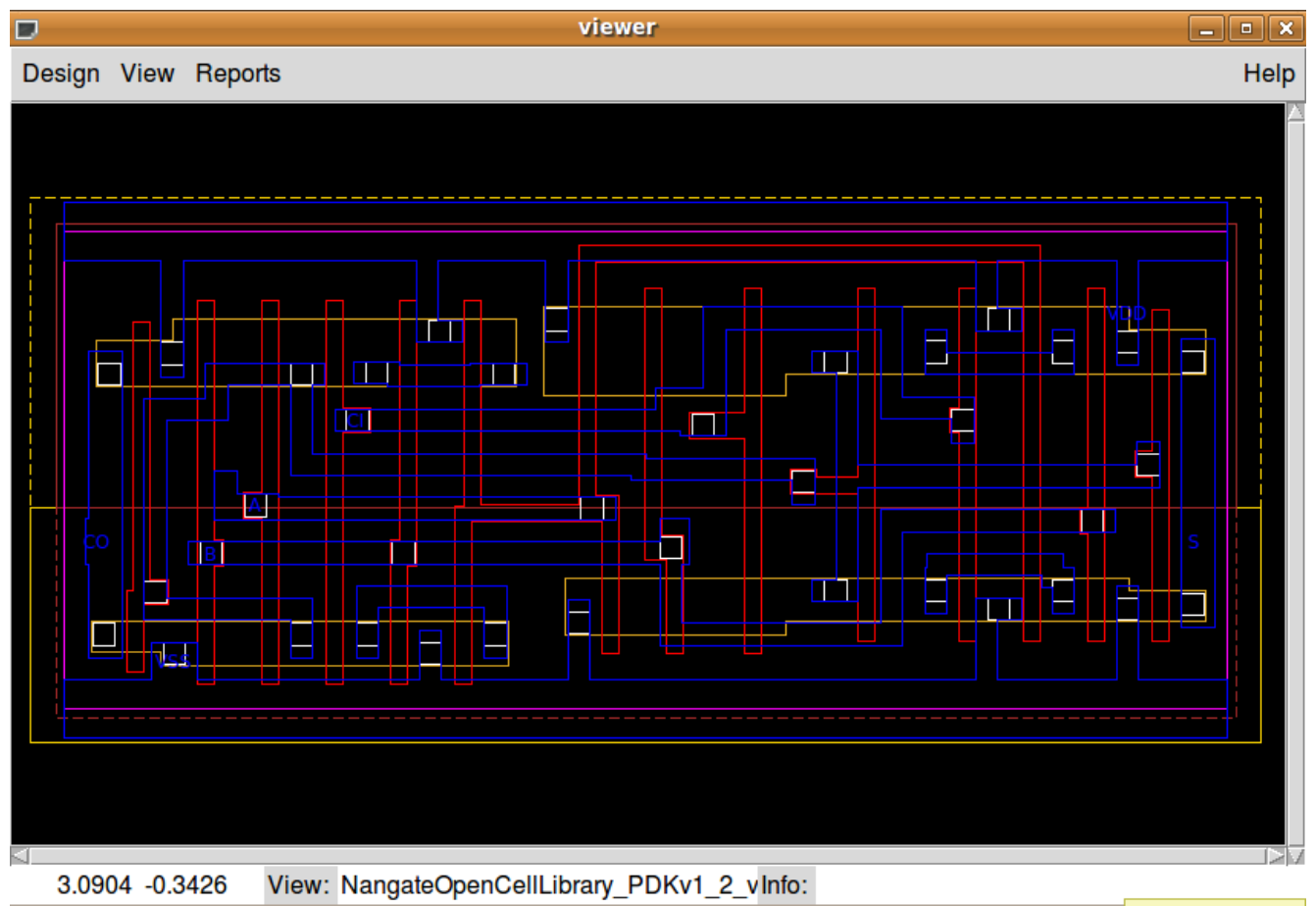

(a) Full Adder Layout - oaViewer

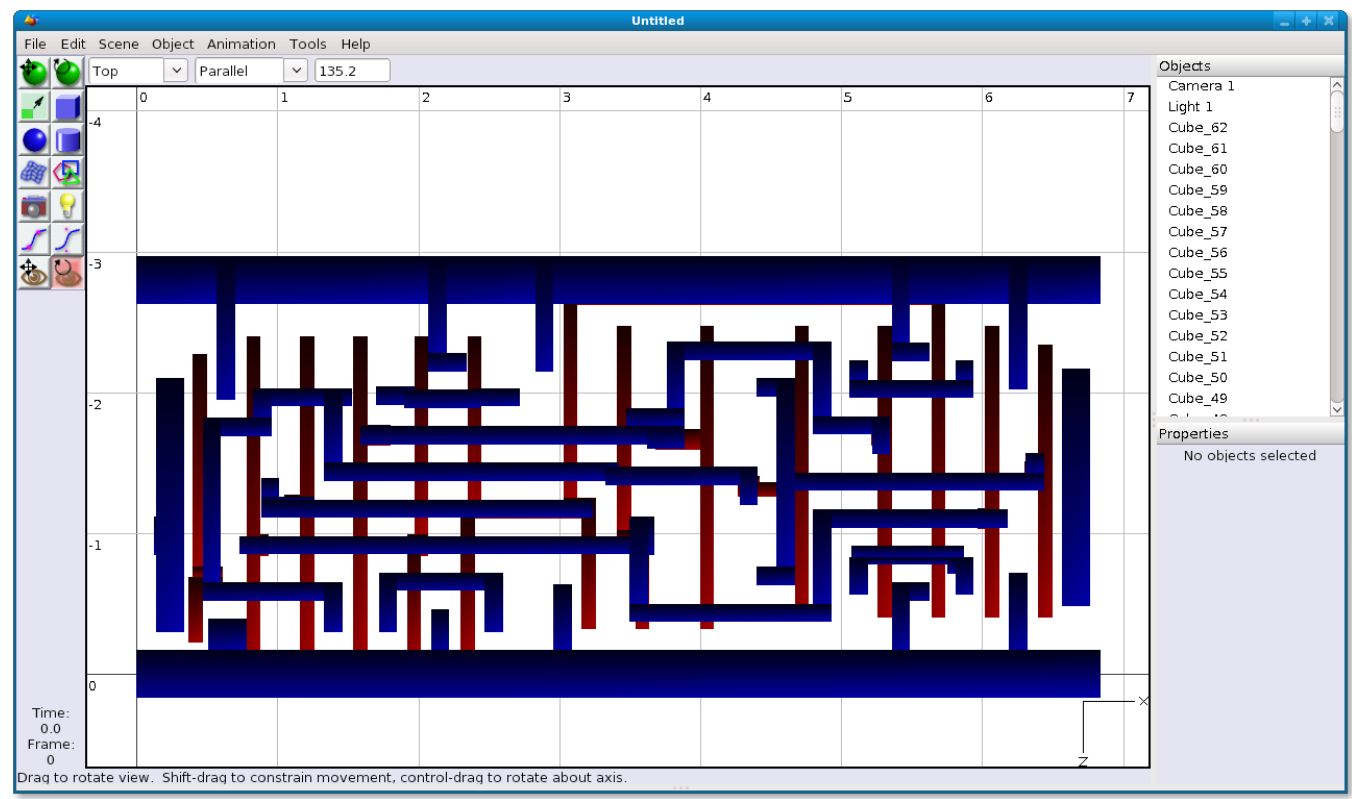

(b) Top View of Full Adder-Art of Illusion

Figure 10.5: Full Adder Cell Viewed in oaViewer and Art of Illusion 


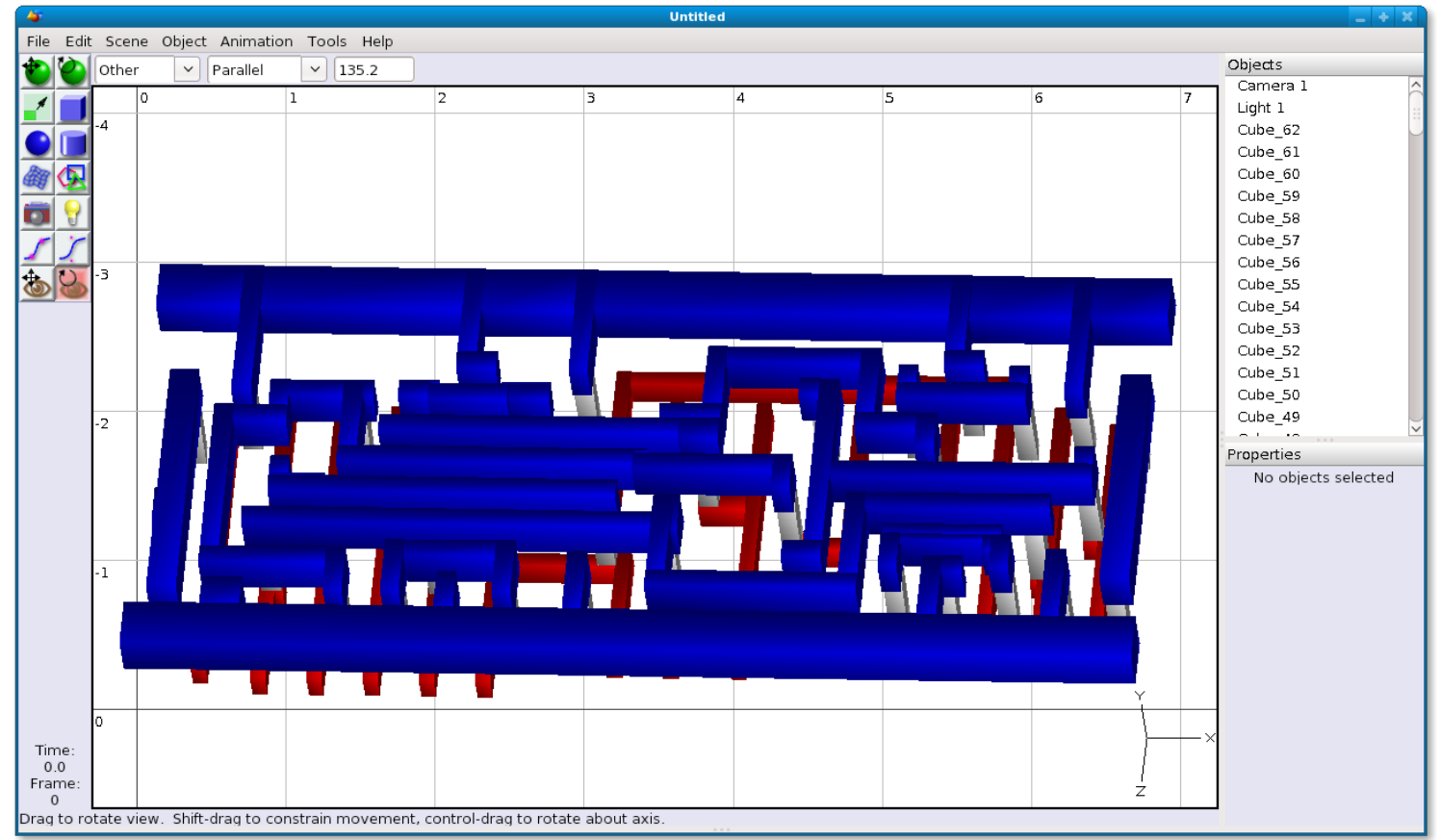

Figure 10.6: 3D view of Full Adder interconnect

Table 10.1: Time to write the .OBJ file

\begin{tabular}{|c|c|c|}
\hline Standard Cell & Time in milli seconds & No. of Polygons \\
\hline NAND2_X2 & 10 & 10 \\
\hline FA_X1 & 20 & 31 \\
\hline
\end{tabular}




\subsection{Limitations of the Algorithm}

The Algorithm presented here to model the IC interconnect three dimensionally has a few limitations. First, from figure 10.6 it can be observed that the heights for both Poly-Metal1 contacts and Source/Drain-Metal1 contacts are the same. This is because the technology database represents both contacts as "contact" in the library. One way to overcome this limitation is by querying the top and bottom layers enclosing the contacts. This type of query can be implemented by using the RegionQuery plugin provided with OpenAccess package. This calls for a complete change in the query algorithm. Also, the algorithm assumes that the interconnect layout comprises of rectangles and rectilinear polygons, whereas Analog/RF IC layouts can have non-orthogonal shapes. 


\section{Chapter 11}

\section{Future work}

The work presented in the document can be extended to make it more efficient and beneficial to the designer and to the design community. In the following sections, a few ideas that were discovered during the course of the research are discussed.

\subsection{Extending OA Database}

In this work, since OpenAccess doesn't model layer thickness, this information was assumed and stored in the translator program. This limits other applications that want to use this information. Hence it is more useful that this information be persistently stored in the database. To accommodate this OpenAccess can be extended to include application defined objects. OpenAccess supports extending Design, Technology, Design Management and Wafer Databases. The data type of these databases can be extended via oaDesignDataType, oaTechDataType, oaDMDataType and oaWaferDataType classes. The classes that are not associated with database data type cannot be extended for e.g, oaFig, oaDesignObject etc. The OpenAccess API documentation lists various classes that can be extended.

There are two different ways to extend OpenAccess. Either by adding an attribute to an existing OA object type, usually called attribute extension, or by 
creating a new object. The later one is called object extension. In both cases the data is stored persistently in the database, i.e, when a design is saved, both the new attributes of the object and the new objects are also stored. The main class that is associated with attribute extension is the oaAppDef class. The oaAppDefObject class can create a new application defined object and the oaAppDef class can set the attributes of that object.

For the work presented in the document the attribute extension can be used to store the layer's thickness information. Database extension is a very useful feature, but it also introduces new set of issues that needs to be addressed. For example, in the case of attribute extensions, if the OA object is deleted, what happens to its attributes?

\subsection{Integration of Parasitic extraction and simulation tools}

Parasitics associated with interconnect have significant impact on a circuit's performance. It would be very useful for the designer if the work presented in the documented can be extended to model the effects of parasitics for Electromigration, IR drops, Clock Skew in clock bus etc., three dimensionally. This requires integrating a parasitic extraction tool, spice simulation tool and an algorithm that models the effect in 3D, with OpenAccess. This section describes how OpenAccess stores the parasitic information and some of the important classes that could be helpful to integrate a parasitic extraction tool with OpenAccess.

OpenAccess supports modeling of these parasitic data as Extracted Design or Annotated Parasitics. In the case of Extracted Design, the parasitics extracted become a part of the design and the simulator can use the new spice netlist 
for re-simulation. This is common in Custom Digital and Analog Design flows. The annotation of parasitic data is commonly used in standard cell based Digital design flows. Here the parasitic data is not part of the design. This is usually done to run simulations faster.

Further, annotated parasitics can either be represented as a Parasitic Network or as a Reduced Model. The parasitic Network, also called Detailed Model representation, results in a detailed parasitic network. The detailed parasitic network consists of resistive, capacitive and inductive elements modeled between the driver and the receiver nodes. This results in a huge parasitic data and can be reduced by using the Reduced Model which makes some useful approximations. A detailed parasitic network can be modeled using oaParasiticNetwork and related classes. Each parasitic network is associated with a particular net. The oaMutuallnductor, oaSeriesRL, oaCouplingCap, oaDiode, oalnductor, oaResistor etc., classes can be used to model parasitic network elements. Each class will have functions associated with them to get or set associated attributes. For e.g, the function oaFloat oaMutuallnductor::getValue(oaAnanlysisPoint* ap) returns the mutual inductance for the analysis point ap. The elements in the network and their values can vary depending on the Analysis Point (PVT condition). The details of creating the network, subnetwork, elements is discussed in [1].

Since Reduced models are approximate models, and aimed at reducing the data, the accuracy of modeling is reduced compared to Detailed models. Lumped Elmore, Pi Elmore and Pi Pole Residue models can be used to represent reduced parasitic information. The oaReducedModel class is the abstract class, that along with oaLumpedElmore, oaPiElmore, oaPiPoleResidue, oaElmore and oaPoleResidue are used to represent parasitics as lumped,pi elements with elmore and pole residue 
delay models. Details on these three types are documented in [1]. Since Annotated parasitics are not owned by the design, the parasitic data will not be loaded by default when the designs are loaded. Parasitics data can be loaded separately.

Once the parasitic information and the simulation data are available, the effect of parasitics on the circuit reliability can be visualized. But finding a parasitic extraction tool that models parasitic data as discussed above can be a challenge. Moreover, integrating spice simulators with OpenAccess requires extending the database. This is because transistor models, primitive resistor, capacitor and inductor elements are not modeled in OA. This can be an arduous task. Also it is very important to note that OpenAccess is just a database that holds information related to the design and manages the relationship between the objects. It is up to the application to compute and generate meaningful results. For e.g, OpenAccess holds the design's netlist information, but it doesn't provide any algorithm to extract the netlist. Extraction is the application's job. 


\section{Chapter 12}

\section{Conclusion}

With technology scaling, the parasitics associated with the IC interconnect have had serious impact on the circuit's performance and reliability. Some of the effects of parasitics include voltage drop and higher current density. As the circuit com-

plexity increases, the designers rely more on the EDA tools to analyze these effects. The main objective of this research was to model voltage and current distribution in the interconnect network three dimensionally using open source tools. As a foundational work, this document presented a tool to visualize the IC interconnect three dimensionally using only free open source software.

The independent contribution of this research is the program that queries the two-dimensional layout data from the design database and converts it into the .OBJ file. Many 3D modeling software read this file format. In addition, the OpenAccess database is relatively new and not much documentation is available. Hence, for designers or students interested in the subject, can use this document and the program as a foundational work.

There exist several disadvantages with the current interoperable models using an intermediate file to exchange designs. OpenAccess is designed to eliminate the use of such intermediate files, by providing an open standard $\mathrm{C}++$ API and 
its reference database implementation. Although OpenAccess was used in this research with an intention to achieve tight integration, an intermediate file format was required. This is because of the time complexity involved in writing a Java wrapper for such a huge $\mathrm{C}++$ API.

Since both the Art of Illusion and the .OBJ file format does not support modeling three-dimensional rectilinear polygons, all the polygons in the design were converted to rectangles. The modified PTR (m-PTR) algorithm to convert orthogonal polygons to rectangles was presented. The rectangles are then modeled as 3D cubes. This turned out to be an advantage since cubes are primitive shapes and rendering cubes is faster than polygons.

The $\mathrm{C}++$ program queries OpenAccess for the layout information and converts the interconnect to 3D objects The program was tested with Nangate's $45 \mathrm{~nm}$ standard cells. These were the only OA designs available in public. The results were then verified with Synopsis oaViewer. Although viewing standard cells was fast and smooth it would be interesting to see the performance with larger designs like an operational amplifier or a PLL. Rendering such large designs is expected to be slower, but fast enough to be able to rotate, pan and zoom in real-time. Besides, Art of Illusion was designed to work with more complex texture, material and illumination models. Hence, AOI should handle the effects of parasitics with various texture and material models well.

As a final thought, most of the layout and schematic editors, 3D viewers, waveform viewers are written in Java. Having a Java wrapper for OA API would not only ease their tight integration with OpenAccess but would also enable them to be integrated with a common open source deployment platform like Eclipse. Having said that one needs to keep in mind that most open source simulators and parasitic 
extractors are written in $\mathrm{C} / \mathrm{C}++$. This makes tight integration of all the different tools in the design flow always challenging.

Overall, the tool presented here, with further enhancement, has a potential to be integrated into the design flow as a part of an open source EDA package. 


\section{References}

[1] M. David, Si2 OpenAccess API Tutorial. Silicon Integration Initiative, Inc, ninth edition ed., 2009.

[2] E. Sicard, "Introduction to microelectronics version 5.1.." Institut National des Sciences Appliquees de Toulouse, 1995.

[3] S. I. Leandro and A. d. L. R. Ricardo, "3d integrated circuit layout visualization using vrml," Future Generation Computer Systems, vol. 17, 2001.

[4] Indrusiak, Reis, and C. Ost, "Jale3d-platform-independent ic/mems layout edition tool," IEEE, 2001.

[5] Si2, OpenAccess API. Si2, 22.04p21 ed., 2009.

[6] K. Gourley and D. Green, "A polygon-to-rectangle conversion algorithm," IEEE Comput. Graph. Appl., vol. 3, no. 1, pp. 31-36, 1983.

[7] W. Neil, H. David, and B. Ayan, CMOS VLSI Design, A Circuits and Systems Perspective. Pearson, 3rd edition ed., 2008.

[8] S. M. Alam, Design tool and methodologies for interconnect reliability analysis in integrated circuits. $\mathrm{PhD}$ thesis, Massachusetts Institute of 
Technology, Cambridge, MA, USA, 2004. Supervisor-Troxel, Donald E. and Supervisor-Thompson, Carl V.

[9] R. Arya, "Chip ir drop reduction through automated via checking and addition," IC Verification and Signoff Mentor Graphics, 2007.

[10] Cadence, "Virtuoso ultrasim full chip simulator netlist based electromigration voltage drop flow." Cadence Design Network, 2007.

[11] Reis, Ribas, and Togni, "Web-based automatic layout generation tool with visualization features."

[12] S. Louis, L. Luciano, and M. Grant, EDA for IC Implementation, Circuit Design and Process Technology, vol. 2. CRC Press, 2006.

[13] M. John, "Api vs. file exchange." http://www.voom.net/api-vs-file-exchange, June 2007.

[14] D. R. Cottrell and T. J. Grebinski, "Interoperability beyond design: Sharing knowledge between design and manufacturing," in ISQED '03: Proceedings of the 4th International Symposium on Quality Electronic Design, (Washington, DC, USA), p. 214, IEEE Computer Society, 2003.

[15] M. Guiney and E. Leavitt, "An introduction to openaccess: an open source data model and api for ic design," in ASP-DAC '06: Proceedings of the 2006 Asia and South Pacific Design Automation Conference, (Piscataway, NJ, USA), pp. 434-436, IEEE Press, 2006.

[16] G. Soumen, "Ic6.x adoption: Creating optimum openaccess designs to enhance designers' productivity," tech. rep., Cadence Design Systems, 2007. 
[17] "Comparison of 3d tools." http://wiki.cgsociety.org/index.php/Comparison_of_3d_tools, 2006.

[18] D. R. Amundson, Python for Open Access 2.2. LSI Logic Corporation.

[19] S. Nahar and S. Sahni, "Fast algorithm for polygon decomposition," Computer-Aided Design of Integrated Circuits and Systems, IEEE Transactions on, vol. 7, pp. 473-483, April 1988.

[20] Y. Lu, Q. Su, and J. Kawa, "An innovative steiner tree based approach for polygon partitioning," in ASP-DAC '08: Proceedings of the 2008 Asia and South Pacific Design Automation Conference, (Los Alamitos, CA, USA), pp. 358-363, IEEE Computer Society Press, 2008.

[21] P. Bourke, "Obj file format." http://local.wasp.uwa.edu.au/p̃bourke/dataformats/obj/.

[22] R. Diane, R. Linda, and T. Lisa, "Mtl material format." http://local.wasp.uwa.edu.au/pbourke/dataformats/mtl/.

[23] Z. Yang and M. Jiang, "Using eclipse as a tool-integration platform for software development," IEEE Softw., vol. 24, no. 2, pp. 87-89, 2007.

[24] J. Amsden, "Eclipse-levels of integration." http://www.eclipse.org/articles/Article-Levels-Of-Integration/levels-ofintegration.html, 2001. 
[25] J. McAffer and J.-M. Lemieux, Eclipse Rich Client Platform: Designing, Coding, and Packaging Java(TM) Applications. Addison-Wesley Professional, 2005.

[26] E. Clayberg and D. Rubel, Eclipse: Building Commercial-Quality Plug-ins (2nd Edition) (Eclipse). Addison-Wesley Professional, 2006.

[27] J. P. Hwang, "Rex - a vlsi parasitic extraction tool for electromigration and signal analysis," in DAC '91: Proceedings of the 28th ACM/IEEE Design Automation Conference, (New York, NY, USA), pp. 717-722, ACM, 1991.

[28] S. M. Rubin, Computer aids for VLSI design. Boston, MA, USA: Addison-Wesley Longman Publishing Co., Inc., 2nd ed., 1994.

[29] M. David, "The shifting sands of dsm characterization," tech. rep., Electronic Design, November 2001.

[30] A. C. Scott, "Cartesian coordinate system." http://en.wikipedia.org/wiki/Cartesian_Coordinate_System, 2002.

[31] Y. Inoue, "Open access overview "industrial experience"," in $A S P-D A C$ '06: Proceedings of the 2006 Asia and South Pacific Design Automation Conference, (Piscataway, NJ, USA), pp. 437-438, IEEE Press, 2006.

[32] M. Guiney, "What is openaccess?" SIGDA Newsl., vol. 35, no. 22, p. 1, 2005.

[33] B.-Z. Chen, H.-M. Chen, L.-D. Huang, and P.-C. Pan, "A stochastic-based efficient critical area extractor on openaccess platform," in GLSVLSI '09: 
Proceedings of the 19th ACM Great Lakes symposium on VLSI, (New York, NY, USA), pp. 197-202, ACM, 2009.

[34] D. Papa, I. L. Markov, and P. Chong, "Utility of the openaccess database in academic research," in ASP-DAC '06: Proceedings of the 2006 Asia and South Pacific Design Automation Conference, (Piscataway, NJ, USA), pp. 440-441, IEEE Press, 2006. 


\section{Appendix A}

\section{.OBJ File Format}

Wavefront Technologies first developed the .OBJ file format for its Advanced Visualizer animation package. This section describes the .obj file format in detail. This will be helpful in modeling the IC interconnect data more accurately. The section is organized as follows:

- Vertex data

- Elements

- Grouping

- Display/render attributes

The following types of data may be included in an .obj file. In this list, the keyword (in the parentheses) follows the data type.

\section{A.1 Vertex data}

It provides coordinates for

- Geometric vertices (v) 
- Texture vertices (vt)

- Vertex normals (vn)

- Parameter space vertices (vp), free-form curve/surface attributes

The vertex data is represented by four vertex lists. A right-hand coordinate system is used to specify the coordinate locations. Syntax: $v$ x y $\mathrm{z}$ w

Specifies a geometric vertex and its $\mathrm{x}, \mathrm{y}$ and $\mathrm{z}$ coordinates. Rational curves and surfaces require a fourth homogeneous coordinate w, also called the weight. $\mathrm{x}$, $\mathrm{y}$ and $\mathrm{z}$ are the $\mathrm{x}, \mathrm{y}$, and $\mathrm{z}$ coordinates for the vertex. These are floating point numbers that define the position of the vertex in three dimensions. $w$ is the weight required for rational curves and surfaces. It is not required for non-rational curves and surfaces. The default is value for $\mathrm{w}$ is1.0.

vp $\mathrm{u} v$ w: specifies a point in the parameter space of a curve or surface. The usage determines how many coordinates are required. For example, Special points for curves require a $1 \mathrm{D}$ control point ( $\mathrm{u}$ only) in the parameter space of the curve and points for surfaces require a $2 \mathrm{D}$ point $(\mathrm{u}$ and $\mathrm{v}$ ) in the parameter spaceof the surface.

vn $i \mathrm{j} k$, specifies a normal vector with components $i, j$, and $k$. Vertex normals affect the smooth-shading and rendering of geometry. For polygons, vertex normals are used in place of the actual face normals. $\mathrm{i}$ and $\mathrm{k}$ are the coordinates for the vertex normal. They are floating point numbers.

vt $\mathrm{u}$ v w, Vertex statement for both polygonal and free-form geometry. Specifies a texture vertex and its coordinates. A $1 \mathrm{D}$ texture requires only u texture coordinates, a 2D texture requires both $\mathrm{u}$ and $\mathrm{v}$ texture coordinates, and a 3D texture requires all three coordinates. $\mathrm{u}$ is the value for the horizontal direction of 
the texture. $\mathrm{v}$ is the value for the vertical direction of the texture and its optional. The default is $0 . \mathrm{w}$ is a value for the depth of the texture.It is also optional. The default is 0 .

\section{A.1.1 Elements}

For polygonal geometry, the element types available in the .obj file are:

- $\operatorname{point}(\mathrm{p})$

- line (1)

- face (f)

Referencing vertex data: For all elements, reference numbers are used to identify geometric vertices, texture vertices, vertex normals, and parameter space vertices. Each of these types of vertices is numbered separately, starting with 1 . This means that the first geometric vertex in the file is 1 , the second is 2 , and so on. The first texture vertex in the file is 1 , the second is 2 , and so on. The numbering continues sequentially throughout the entire file. Frequently, files have multiple lists of vertex data. This numbering sequence continues even when vertex data is separated by other data.

Referencing groups of vertices: Some elements, such as faces and surfaces, may have a triplet of numbers that reference vertex data. These numbers are the reference numbers for a geometric vertex, a texture vertex, and a vertex normal. Each triplet of numbers specifies a geometric vertex, texture vertex, and vertex normal. The reference numbers must be in order and must be separated by slashes $(/)$. 
- The first reference number is the geometric vertex.

- The second reference number is the texture vertex. It follows the first slash.

- The third reference number is the vertex normal. It follows the second slash. The following is a portion of a sample file for a four-sided face element:

f $1 / 1 / 12 / 2 / 23 / 3 / 34 / 4 / 4$

Using $\mathrm{v}$, vt, and vn to represent geometric vertices, texture vertices, and vertex normals, the face statement would read: $\mathrm{f} \mathrm{v} / \mathrm{vt} / \mathrm{vn} \mathrm{v} / \mathrm{vt} / \mathrm{vn} \mathrm{v} / \mathrm{vt} / \mathrm{vn} \mathrm{v} / \mathrm{vt} / \mathrm{vn}$

If there are only vertices and vertex normals for a face element (no texture vertices), you would enter two slashes (//). For example: f $1 / / 12 / / 23 / / 3$ $4 / / 4$

The following syntax statements are listed in order of complexity of geometry. p v1 v2 v3 . . ., Specifies a point element and its vertex. Multiple points can be specified with this statement. $\mathrm{v}$ is the vertex reference number for a point element. Each point element requires one vertex.

$1 \mathrm{v} 1 / \mathrm{vt} 1 \mathrm{v} 2 / \mathrm{vt} 2 \mathrm{v} 3 / \mathrm{vt} 3 . . \quad$. Specifies a line and its vertex reference numbers. Optionally the texture vertex reference numbers can be included. There is no space between the number and the slash. $\mathrm{v}$ is a reference number for a vertex on the line. Minimums of two vertex numbers are required. There is no limit on the maximum. vt is an optional argument. vt is the reference number for a texture vertex in the line element. It must always follow the first slash.

f v1/vt1/vn1 v2/vt2/vn2 v3/vt3/vn3 ... Specifies a face element and its vertex reference number. The texture vertex and vertex normal reference numbers can also be included. The reference numbers for the vertices, texture vertices, and vertex normals must be separated by slashes $(/)$. There is no space between 
the number and the slash. $\mathrm{v}$ is the reference number for a vertex in the face element. Minimum of three vertices are required. vt is an optional argument. vt is the reference number for a texture vertex in the face element. It always follows the first slash. vn is an optional argument. vn is the reference number for a vertex normal in the face element. It must always follow the second slash. Face elements use surface normals to indicate their orientation. If vertices are ordered counterclockwise around the face, both the face and the normal will point toward the viewer. If the vertex ordering is clockwise, both will point away from the viewer. If vertex normals are assigned, they should point in the general direction of the surface normal, otherwise unpredictable results may occur. If a face has a texture map assigned to it and no texture vertices are assigned in the f statement, the texture map is ignored when the element is rendered.

\section{A.1.2 Grouping}

There are four statements in the obj file to help you manipulate groups of elements:

- group name (g), Group name statements are used to organize collections of elements and simplify data manipulation for operations in the Model.

- smoothing group (s), Smoothing group statements let you identify elements over which normals are to be interpolated to give those elements a smooth, non-faceted appearance. This is a quick way to specify vertex normals.

- merging group (mg), Merging group statements are used to identify free-form elements that should be inspected for adjacency detection. Merging groups can also be used to exclude surfaces, which are close enough to be considered adjacent but should not be merged. 
- object name (o), Object name statements let you assign a name to an entire object in a single file.

All grouping statements are state-setting. This means that once a group statement is set, it applies to all elements that follow until the next group statement.

Syntax: g group-name1 group-name2...., Specifies the group name for the elements that follow it. There can be multiple group names. If there are multiple groups on one line, the data that follows belong to all groups. Group information is optional. group-name is the name for the group. Letters,numbers, and combinations of letters and numbers are accepted for group names. The default group name is default.

\section{A.1.3 Display/Render attributes}

Display and render attributes describe how an object looks when displayed. All display and render attribute statements are state-setting.

- material name (usemtl)

- material library (mtllib)

- shadow casting (shadow_obj)

- ray tracing (trace_obj)

Syntax:

usemtl material_name Specifies the material name for the element following it. Once a material is assigned, it cannot be turned off; it can only be changed. material_name is the name of the material. If a material name is not specified, a white material is used. 
mtllib filename1 filename2..., Specifies the material library file for the material definitions set with the usemtl statement. You can specify multiple filenames with mtllib. If multiple files are specified, the first file listed is searched first for the material definition; the second file is searched next, and so on.

shadow_obj filename Specifies the shadow object filename. This object is used to cast shadows for the current object.

trace_obj filename, specifies the ray tracing object filename. This object will be used in generating reflections of the current object on reflective surfaces. Ray tracing can greatly increase the rendering time. filename is the filename for the ray tracing object. The object file can be an .obj or .mod file. If a filename is given without an extension, an extension of obj is assumed. 


\section{Appendix B}

\section{.MTL File Format}

An .mtl file is typically organized as shown below.

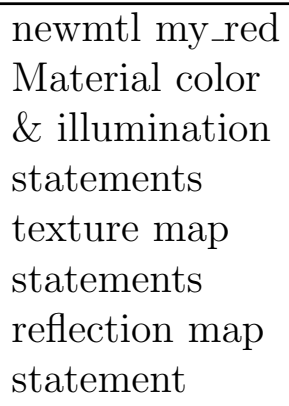

Each material description in a .mtl file consists of the newmtl statement, which assigns a name to the material and designates the start of a material description. The material color, texture map, and reflection map statements that describe the material follow this statement. An .mtl file map contains many different material descriptions. The following is a sample format for a material definition in an .mtl file:

Material Name: The material name statement assigns a name to the material description. The following syntax describes the material name statement.

newmtl name, Specifies the start of a material description and assigns a name to the material. An .mtl file must have one newmtl statement at the start of each 
Material name statement:

newmtl my_mtl

Material color and illumination statements:

Ka 0.04350 .04350 .0435

Kd 0.10860 .10860 .1086

Ks 0.00000 .00000 .0000

illum 6

material description. "name" is the name of the material. Names may be any length but cannot include blanks. Underscores may be used in material names.

Material color and illumination: The statements in this section specify color, transparency, and reflectivity values.The following syntax describes the material color and illumination statements that apply to all .mtl files.

Ka r g b

Ka spectral file.rfl factor

Ka xyz x y z

To specify the ambient reflectivity of the current material, the "Ka" statement can be used, the "Ka spectral" statement, or the "Ka xyz" statement.

Ka $r \mathrm{~g}$ b, The Ka statement specifies the ambient reflectivity using RGB values. "r g b" are the values for the red, green, and blue components of the color. The $\mathrm{g}$ and $\mathrm{b}$ arguments are optional. If only $\mathrm{r}$ is specified, then $\mathrm{g}$, and $\mathrm{b}$ are assumed to be equal to $\mathrm{r}$. The $\mathrm{r} g \mathrm{~b}$ values are normally in the range of 0.0 to 1.0 . Values outside this range increase or decrease the reflectivity accordingly.

Ka spectral file.rfl factor, The "Ka spectral" statement specifies the ambient reflectivity using a spectral curve. "file.rfl" is the name of the .rfl file. "factor" is an optional argument. "factor" is a multiplier for the values in the .rfl file and defaults to 1.0 , if not specified. 
Ka xyz x y z, The "Ka xyz" statement specifies the ambient reflectivity using CIEXYZ values. "x y z" are the values of the CIEXYZ color space. The y and $\mathrm{z}$ arguments are optional. If only $\mathrm{x}$ is specified, then $\mathrm{y}$ and $\mathrm{z}$ are assumed to be equal to $\mathrm{x}$. The $\mathrm{x} \mathrm{y} \mathrm{z}$ values are normally in the range of 0 to 1 . Values outside this range increase or decrease the reflectivity accordingly.

To specify the diffuse reflectivity of the current material, the "Kd" statement, the "Kd spectral" statement, or the "Kd xyz" statement can be used.

Kd r g b

Kd spectral file.rfl factor

Kd xyz x y z

To specify the specular reflectivity of the current material, the "Ks" statement, the "Ks spectral" statement, or the "Ks xyz" statement can be used.

Ks r g b

Ks spectral file.rfl factor

Ks xyz x y z

The "illum" statement specifies the illumination model to use in the material. Illumination models are mathematical equations that represent various material lighting and shading effects.

illum illum number

"illum number" can be a number from 0 to 10. 


\section{Appendix C}

\section{Importing OpenAccess to Eclipse}

Following is the procedure to install OpenAccess from the source code using Eclipse. The OA source code used is DM4 22_04p021, on Linux (Fedora Core 10) with gcc version 4.3. The code was successfully compiled in Eclipse 3.4 with CDTv5 plugin. CDT plugin is $\mathrm{C} / \mathrm{C}++$ Development Tool plugin, which is required to compile $\mathrm{C} / \mathrm{C}++$ source code.

1. Extract the OA source code into a folder. For example, /home/username/OpenAccess/src/

2. Create a new $\mathrm{C}++$ project in Eclipse, uncheck use default location and point to the source directory. In this case/home/username/OpenAccess/src/

3. Enter a project name and select Executables $\rightarrow$ Empty Project

4. In the next step, there will be two configurations, Debug and Release, ensure both of them are checked and click Finish.

5. In the Eclipse workbench $\rightarrow$ Project Explorer, will list the OA project with OA source code.

6. For some reason Eclipse doesnt recognize the 'make' targets already in the 
Makefile, so a new make target has to be specified for Eclipse. To compile core OA, "install_oa" target needs to be created.

7. Under project explorer window, Right click on the "oa" folder, select Make Targets $\rightarrow$ create, specify "install_oa" for both Target Name and Make Target and specify 'make OPTMODE=dbg' for Build command in Debug configuration. To build in Release configuration specify 'make OPTMODE=opt' for Build command.

8. To build the project, Right Click on OA Project $\rightarrow$ Make Targets $\rightarrow$ Build, and select install_oa, click on Build to compile OpenAccess.

9. Some times it might be required to set COMPILER_PATH environmental variable to appropriate $\mathrm{GCC}$ and $\mathrm{G}++$ compiler path.

By default OpenAccess looks for compiler in /opt/gcc4.1.1/bin/gcc and/opt/gcc4.1.1/bin/g++.

This can be changed by editing the /oa_source_directory/build/make/linux_rhel30_gcc411_32.variables, file.

\section{C.1 Installing TCL wrappers}

Installing OpenAccess TCL wrappers, require tcl version 8.4.6 or above. Following are the steps to install TCL wrappers using Eclipse and uses tcl 8.5 version. OpenAccess Tcl wrapper require tcl.h header file, which mean Tcl development version is also required. It is assumed that the base tcl installation directory is /usr. One needs to edit few files in OpenAccess before installing Tcl wrappers.

1. Edit the dep.variables file, under Project Explorer $\rightarrow$ build $\rightarrow$ make and 
look for TCL version and change it to the version installed, in this case 8.5. Also change TCL_HOME to / usr

2. Edit the GNUMakef ile file, under Project Explorer $\rightarrow$ oaLang $\rightarrow$ test $\rightarrow$ app and look for PROG_LDLIBS and replace -ltcl8.4 with -L /TCL_HOME/lib ltcl 8.5

3. Right click on the "oa" folder, select Make Targets $\rightarrow$ create, specify "install_lang" for both Target Name and Make Target and specify make OPT$\mathrm{MODE}=\mathrm{dbg}$ for the Build command in Debug configuration. To build in Release configuration specify make OPTMODE=opt for the Build command.

4. To install TCL wrappers, Right Click on the Project $\rightarrow$ Make Targets $\rightarrow$ Build, and select install_lang, click on Build to install TCL wrappers. 


\section{Appendix D}

\section{Importing Art of Illusion to Eclipse}

Below are the steps describing the procedure to install Art of Illusion in Eclipse. Again version of Eclipse used is 3.4 and Art of Illusion Source code is 2.7.1.

1. Extract Art of Illusion source code to a directory e.g, /home/Artoftllusion/

2. Create a new project in Eclipse, by selecting New $\rightarrow$ Project $\rightarrow$ Java $\rightarrow$ Java Project From an existing Ant Build File

3. Next Navigate to Artoflllusion source directory, i.e, /home/Artoflllusion and select ArtOflllusion.xml file, the project name and Ant build is selected automatically and click 'Finish'.

4. This will import the necessary libraries and source to project explorer. However the jmf.jar file needed for animation is not included in the source and can be linked as an external library.

5. Now right click on ArtofIllusion.xml $\rightarrow$ Run As $\rightarrow$ Ant Build. This will compile the source code and creates the ArtOfIllusion.jar file.

6. To run Art of Illusion from Eclipse, right click on the project $\rightarrow$ Run As $\rightarrow$ Java Application, now select ArtOffllusion.artofillusion. 


\section{Appendix E}

\section{Schema Diagram Legend}

The following figure shows the legend for reading OpenAccess API information model schema diagrams[5]. 


\section{Colors of classes in schema diagrams}

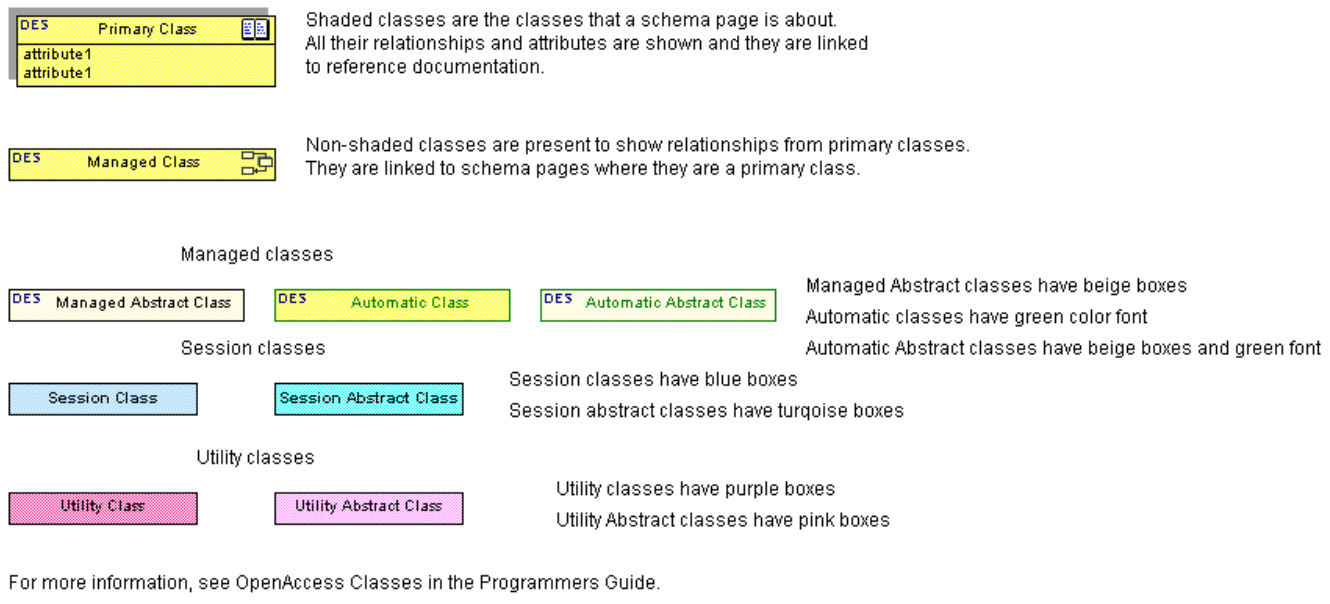

\section{Relationship between objects}

The number of to objects that are related to a single from object is shown in the arrows next to the to class in the legend below as follows

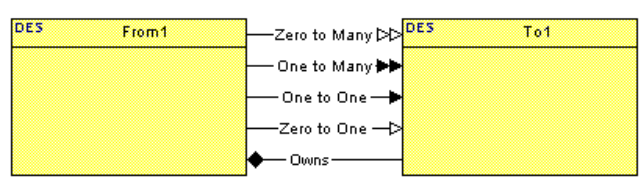

The class with the black diamond owns the other class it has a relationship to so that if the owner is deleted, the owned objects get deleted implicitly.

Inheritance between classes

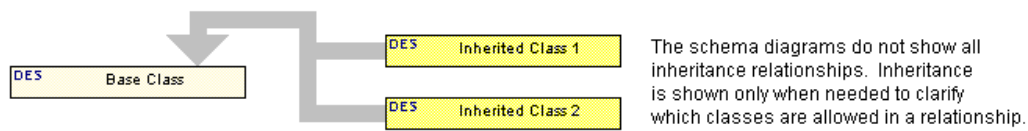

Background colors used in schema diagrams

\begin{tabular}{|c|c|c|}
\hline DES Design DB class & \multicolumn{2}{|c|}{$\begin{array}{l}\text { Classes that are part of the Design database are shown with a light yellow } \\
\text { background and have DES at the top left corner. }\end{array}$} \\
\hline Tech DB class & \multicolumn{2}{|c|}{$\begin{array}{l}\text { Classes that are part of Technology database are shown with a peach } \\
\text { background and have } \mathrm{TECH} \text { at the top left corner. }\end{array}$} \\
\hline DM class & \multicolumn{2}{|c|}{$\begin{array}{l}\text { Classes that are part of Design Management database are shown with a green } \\
\text { background and have DM at the top left corner. }\end{array}$} \\
\hline WAF wafer DB class & \multicolumn{2}{|c|}{$\begin{array}{l}\text { Classes that are part of the Wafer database are shown with a purple } \\
\text { background and have WAF at the top left corner. }\end{array}$} \\
\hline oaConstraint & \multicolumn{2}{|c|}{ Classes that are valid across all databases have a blue background. } \\
\hline & $\frac{1}{v}$ & When there is a relationship between objects \\
\hline Design DB class & DES Another Design DB class & $\begin{array}{l}\text { in two different cellviews, the two objects } \\
\text { are shown in separate background boxes. }\end{array}$ \\
\hline
\end{tabular}

